\title{
Chemical interactions between Verticillium longisporum and oilseed rape Brassica napus
}

\author{
Dissertation \\ to obtain the Ph. D. degree \\ in the International Ph. D. Program for Agricultural Sciences in Goettingen (IPAG) \\ at the Faculty of Agricultural Sciences, \\ Georg-August-University Göttingen, Germany
}

\author{
presented by \\ Husam Ibrahem Aroud \\ born in Damascus, Syria
}

Göttingen, May 2013 
D7

1. Name of supervisor: Prof. Dr. Petr Karlovsky

2. Name of co-supervisor: Prof. Dr. Andreas von Tiedemann

Date of dissertation: 03.07.2013 


\section{Table of Contents}

Chapter 1: General Introduction.........................................................................................

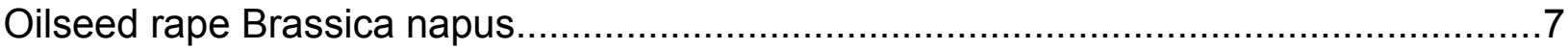

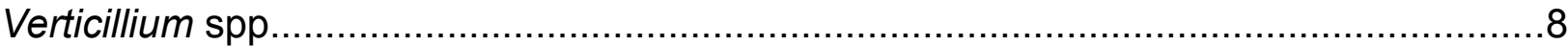

Verticillium longisporum ................................................................................... 10

Interactions between $V$. longisporum and $B$. napus...........................................13

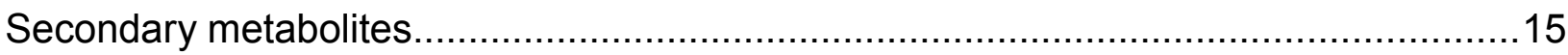

Plant secondary metabolites............................................................... 15

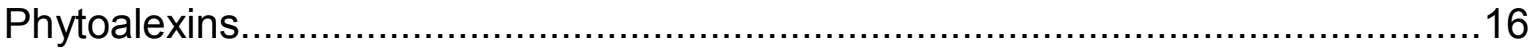

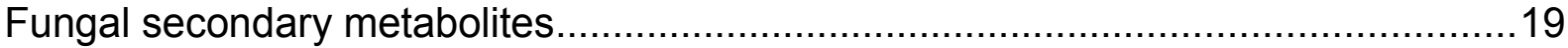

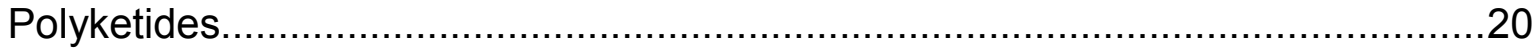

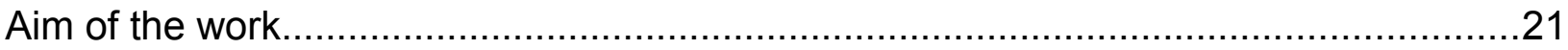

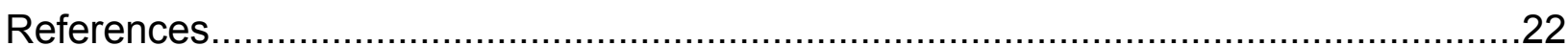

Chapter 2: Isolation and purification of secondary metabolites from xylem sap of Brassica napus infected with Verticillium Iongisporum...............................................31

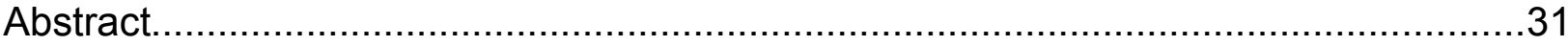

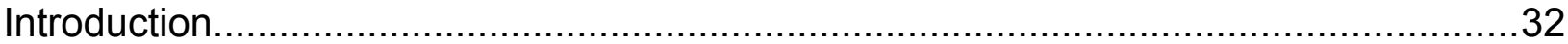

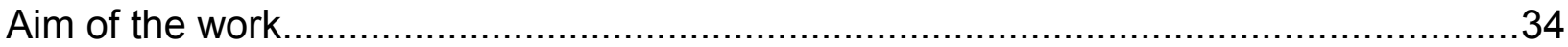

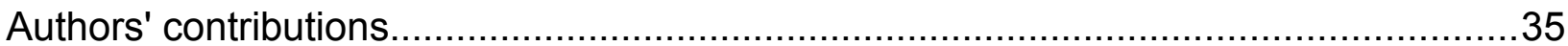

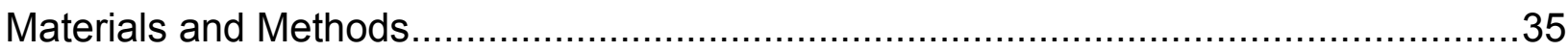

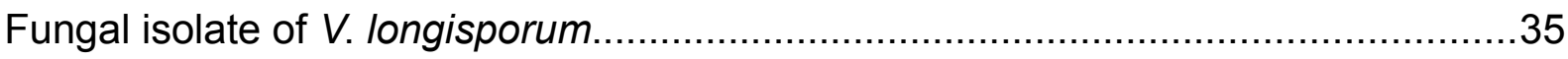

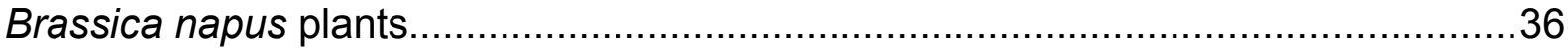

Preparation of Verticillium longisporum inoculum.............................................36

Inoculation of the seedlings with Verticillium longisporum ....................................

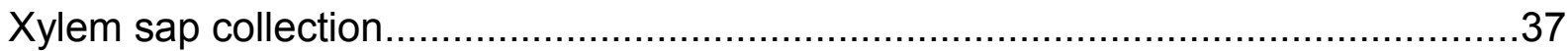

High performance liquid chromatography and mass spectrometry...................... 37

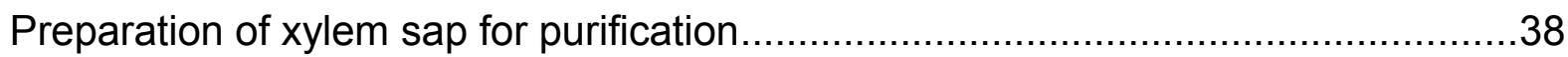

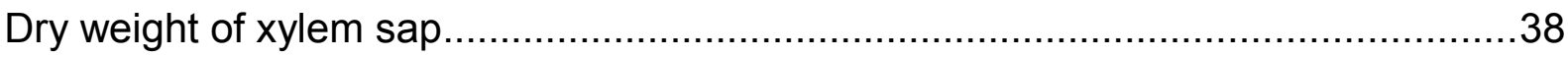

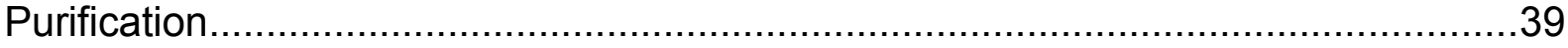

First step of purification with preparative HPLC .................................................39

UPLC combined with PDA-TOF MS Detection............................................40

Second step of purification (Semi-preparative purification) ..............................41

Results 


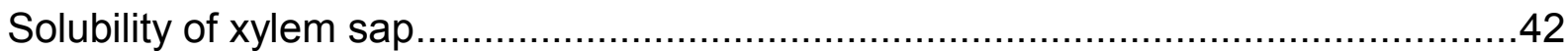

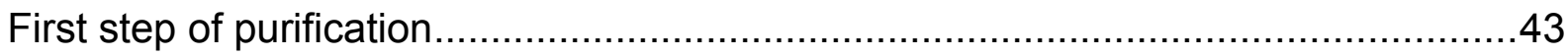

UPLC combined with PDA-TOF- MS Detection of pooled fractions (Sample P) ....48 Second step of purification (Semi-preparative purification)....................................50

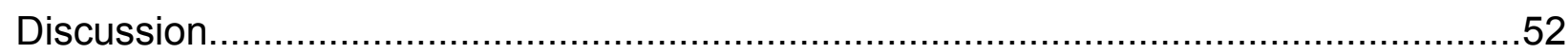

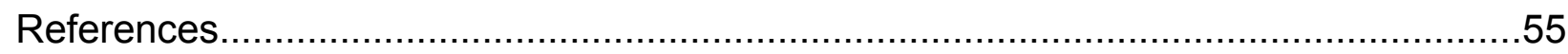

Chapter 3: The effect of Verticillium longisporum infection on the growth of Agrobacterium tumefaciens and Saccharomyces cerevisiae in xylem sap of

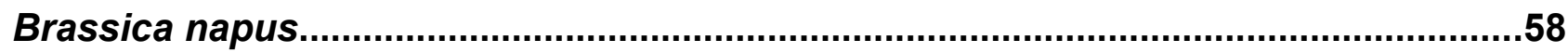

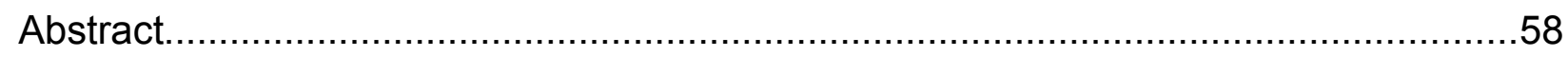

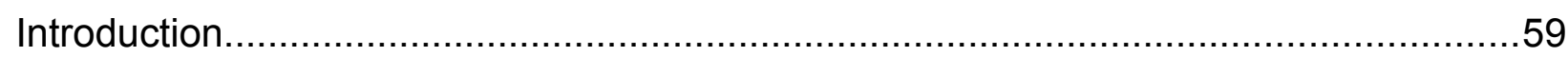

Aim of the work

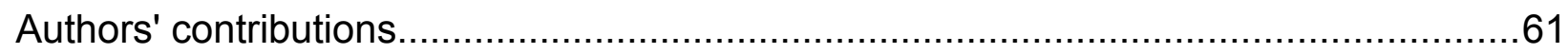

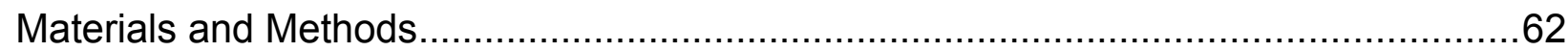

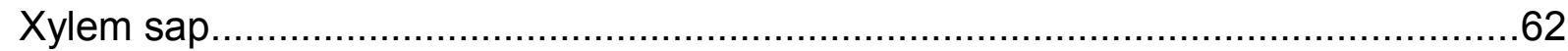

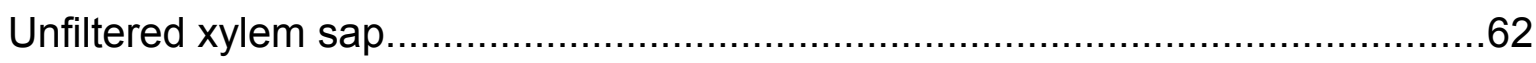

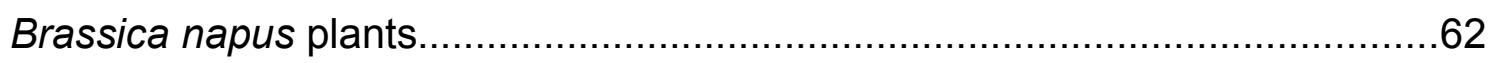

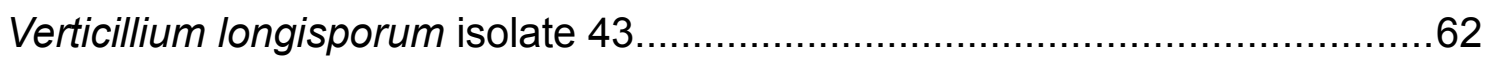

Preparation of Verticillium longisporum inoculum............................................63

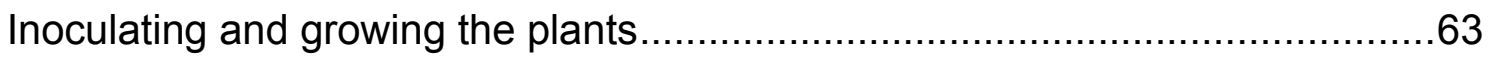

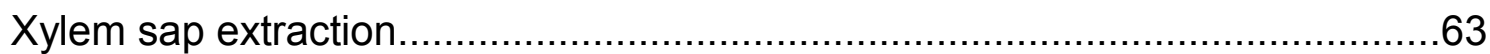

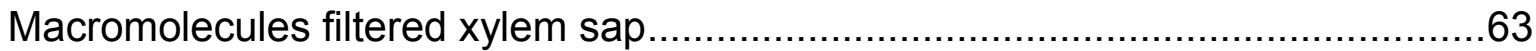

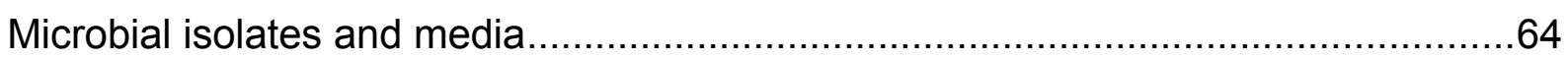

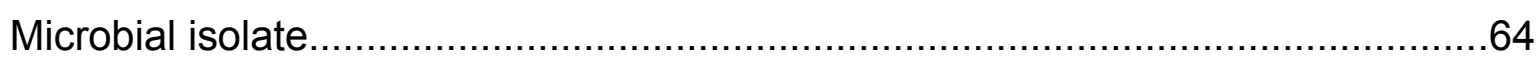

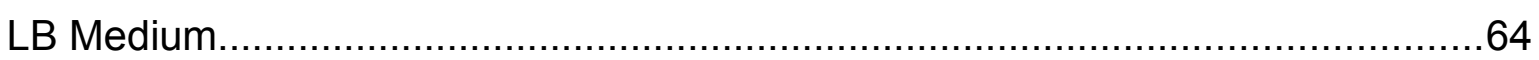

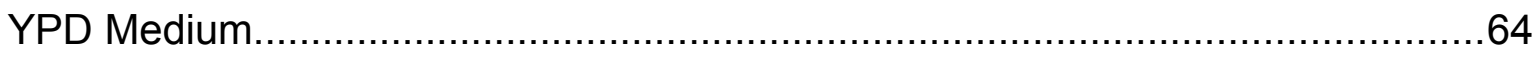

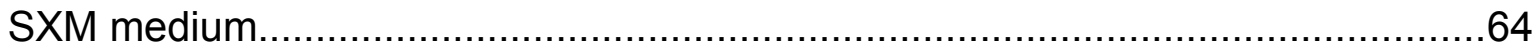

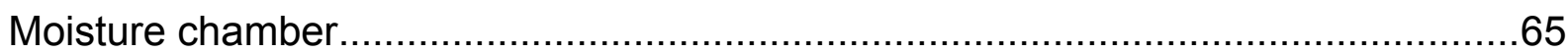

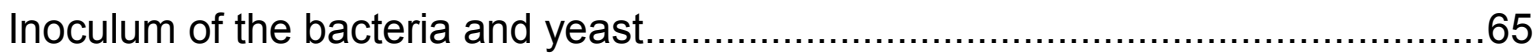

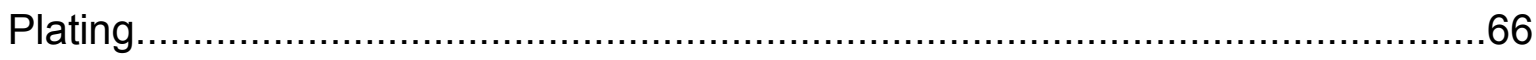

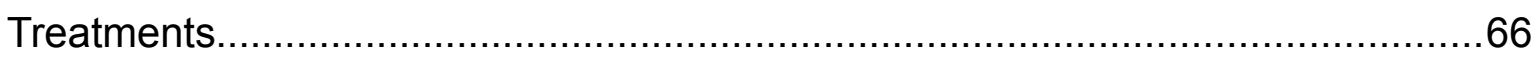

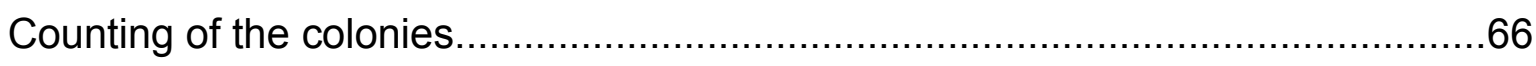

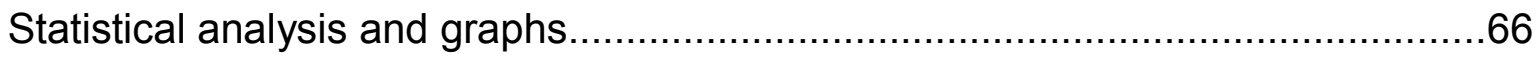

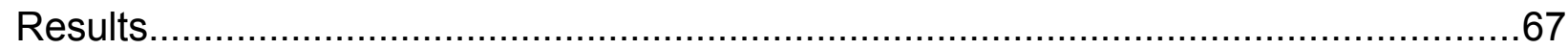


Bioassay on the bacteria Agrobacterium tumefaciens........................................67

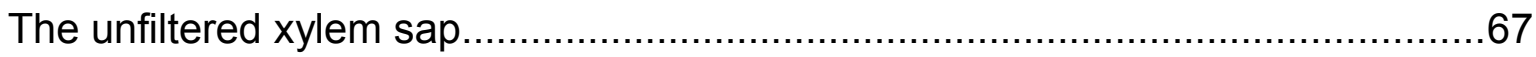

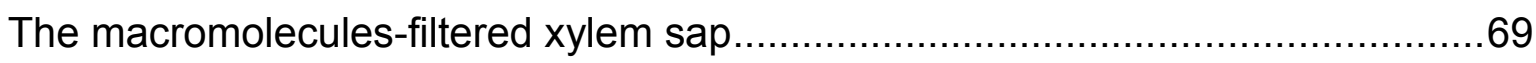

Bioassay on the yeast Saccharomyces cerevisiae............................................69

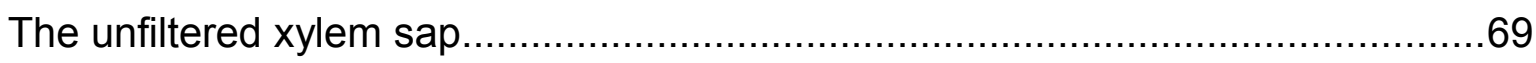

The macromolecules-filtered xylem sap.............................................. 72

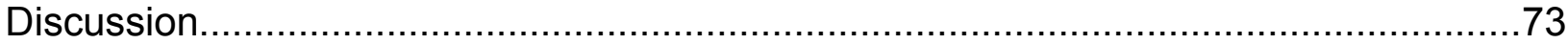

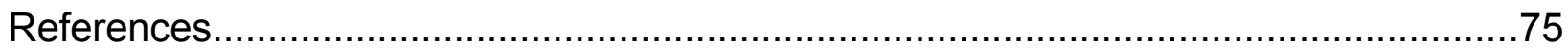

Chapter 4: Comparative metabolic profiling of wild type and polyketide synthase silenced strain of Verticillium Iongisporum .............................................................79

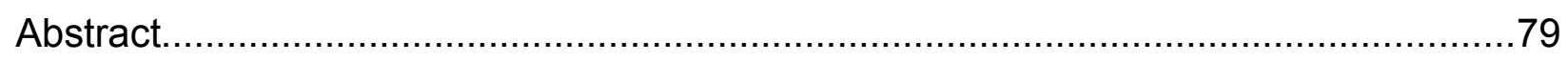

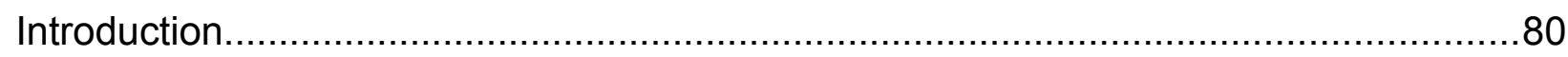

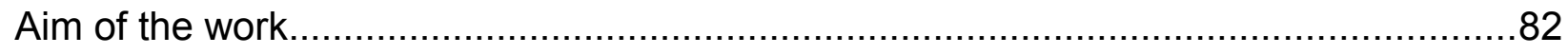

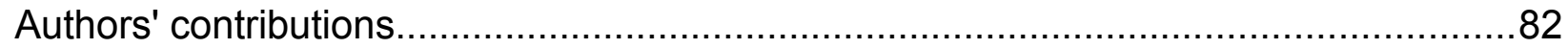

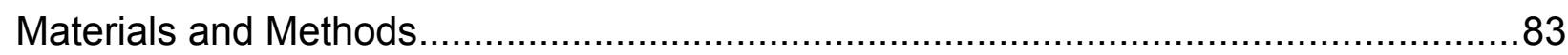

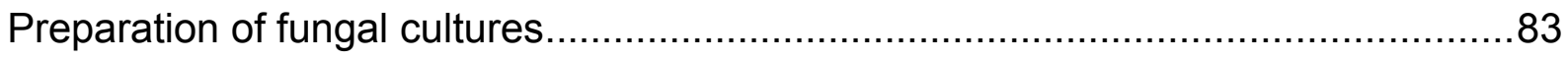

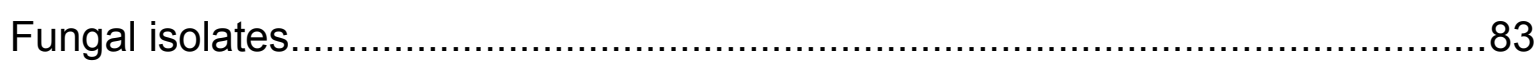

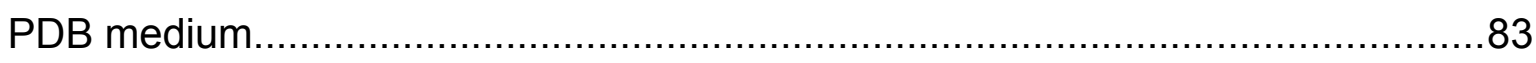

Inoculation of PDB media and preparation of fungal cultures..........................83

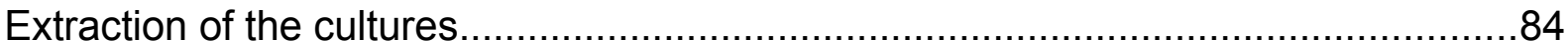

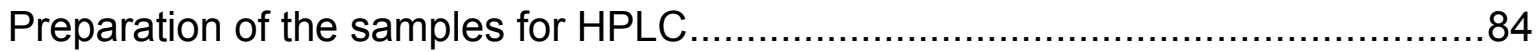

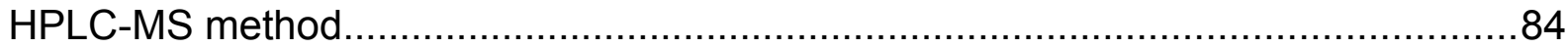

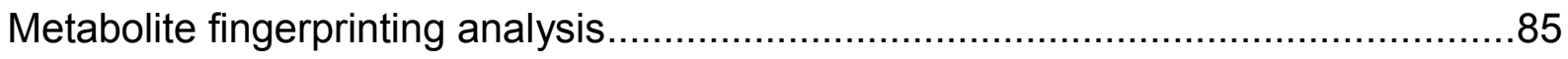

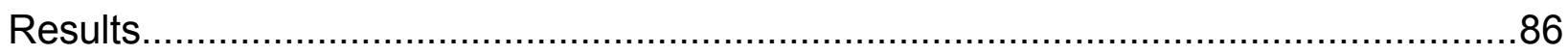

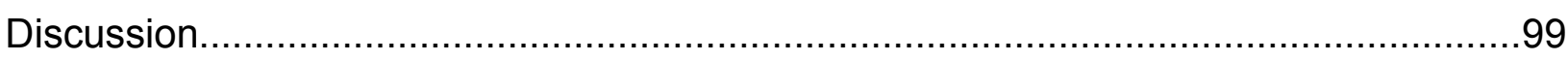

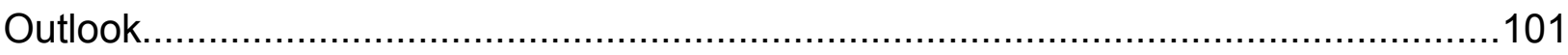

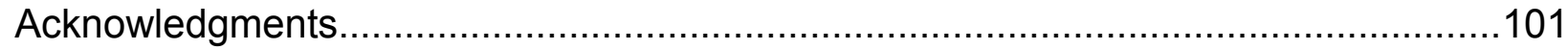

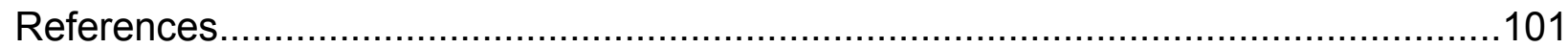

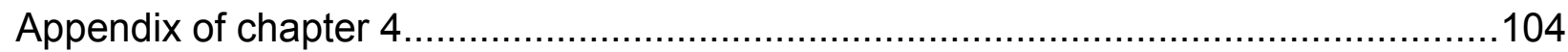

Chapter 5: General discussion...........................................................................107

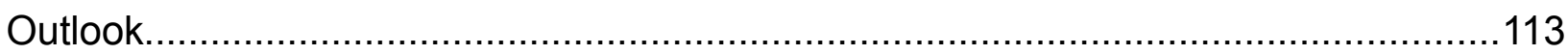

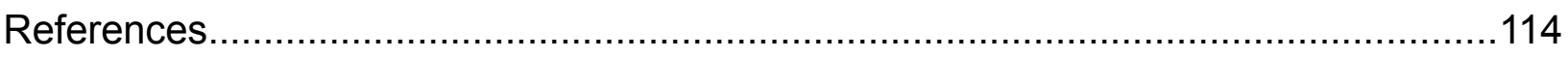

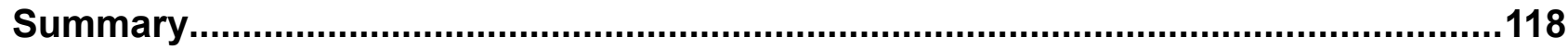

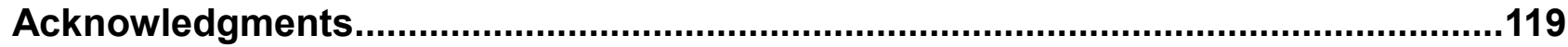


Content

Curriculum vitae.............................................................................................120

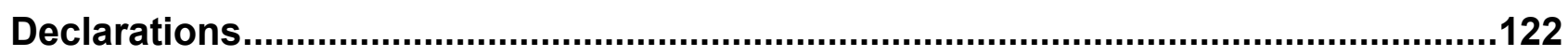




\section{Chapter 1: General Introduction}

\section{Oilseed rape Brassica napus}

The genus Brassica belongs to the plant family Brassicaceae which contains hundreds of genera and thousands of species, including many economically important cultivated crops in different parts of the world, which have been domesticated for many centuries and consumed as vegetables, condiments or oil seeds with high nutritional value and health-giving additives (Katz and Weaver, 2003). Furthermore some of them have scientific importance like Arapidopsis thaliana and Thellungiella salsuginea. This economic and scientific importance has been resulting in hundreds of published scientific articles about crucifers every year (Amtmann, 2009; Katz and Weaver, 2003; Pedras and Yaya, 2010).

Oilseed rape (Brassica napus L.) is one of the most important oilseed crops in the world and is widely cultivated in Canada, China and Europe. According to the United States Department of Agriculture report in April 2013, oilseed rape is the second major oilseed crop produced with a global production of 60.93 million metric tons, and is the third source of vegetable oil globally with a production of 23.91 million metric tons (USDA, 2013). In Europe, oilseed rape is considered the most important oilseed crop mainly cultivated in Northern Europe, with an increasing cultivation area due to its utilization for bio-fuel production, also as vegetable oil and animal fodder. Fig. 1 shows the increasing area of cultivation of oilseed rape in Europe (FAO, 2013). There have always been efforts to improve the yield of oilseed rape in Europe (Diepenbrock, 2000). However, soil-borne diseases pose a major threat to oilseed rape yields, especially in areas with tight oilseed rape crop rotations leading to inoculum accumulation in the soil and potential yield losses in the following oilseed crops (Dunker et al., 2008). In recent decades Verticillium diseases have become a major challenge for the cultivation of oilseed rape and closely related Brassica species in different parts of the world including Europe, Asia and North America. In Canada, Verticillium disease can be the major potential threat to the production of oilseed rape (Heale and Karapapa, 1999). Since 1980 Verticillium disease on oilseed rape has been a major constraint for oilseed crop cultivation in Northern Europe, 
particularly in Sweden, Germany, Poland and France (Fahleson et al., 2004; Johansson et al., 2006; Steventon et al., 2002).

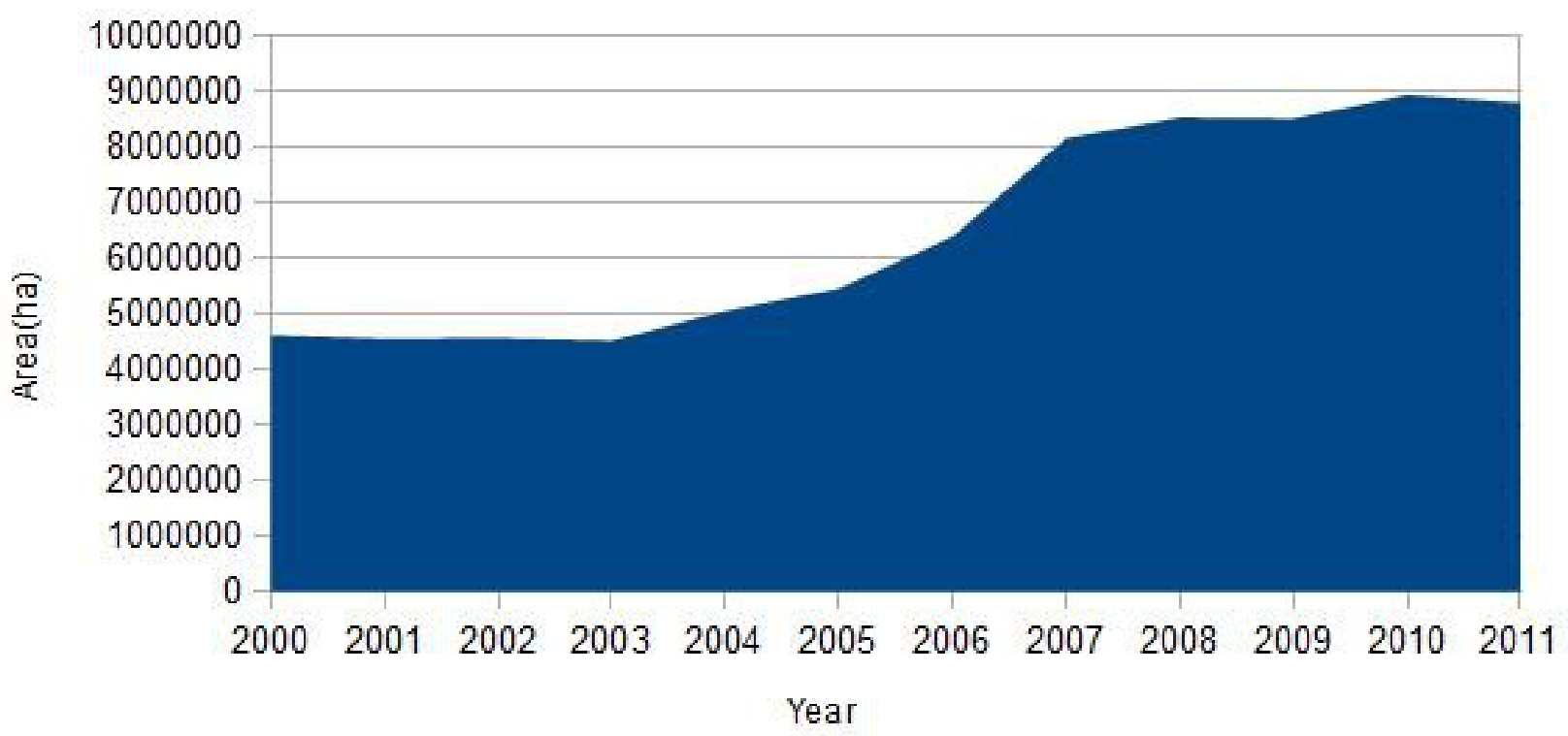

Figure 1. Area harvested with oilseed rape in Europe (Source: FAOSTAT)

\section{Verticillium spp.}

The genus Verticillium comprises a widespread group of Ascomycetes, including soil borne plant pathogenic species which cause vascular wilt diseases on plants especially in the temperate and subtropical regions of the world (Fradin and Thomma, 2006; Pegg and Brady, 2002). Estimated yield losses in many important crops around the world, due to Verticillium diseases; can reach billions of dollars yearly (Pegg and Brady, 2002). Diseases caused by Verticillium species are often referred to as Verticillium wilt disease because of the most typical symptom caused by theses species (Pegg and Brady, 2002). Besides wilt, symptoms of Verticillium infection on plants can include retarded growth, chloroses on the leaves or necrotic lesions. Brown discoloration of the vascular system, can be revealed by crosssections of the vascular tissue of infected plants (Fradin and Thomma, 2006). This phenomenon may be attributed to the accumulation of phenolic substances which play a role 
in the plants resistance response to the disease (Eynck et al., 2009; Obermeier et al., 2013).The number of phytopathogenic species of the genus Verticillium is controversially discussed (Beinhoff, 2011). According to (Barbara and Clewes, 2003), there are six plant pathogenic Verticillium species: $V$. dahliae isolates which form microsclerotia, $V$. albo-atrum including most of isolates which only produce dark resting mycelium (excluding isolates which produce the mycelium in bundles) and four species covering saprophytes and non-pathogenic species ( $V$. nigrescens, $V$. nubilum, $V$. tricorpus and $V$. theobromae). Recent studies assigned $V$. nigrescens to the genus Gibellulopsis, and $V$. theobromae to the genus Musicillium, therefore the number of plant pathogenic species of the genus Verticillium was reduced to four (Zare et al., 2007). Generally it is difficult to distinguish the causal agents of Verticillium diseases and molecular techniques are often needed to differentiate among them (Fahleson et al., 2004; Fradin and Thomma, 2006; Nazar et al., 1991; Pérez-Artés et al., 2000).The most common plant pathogenic species are $V$. dahliae and $V$. albo-atrum. While $V$. albo-atrum is considered to be a major disease on alfa alfa (Aubé and Sackston, 1964), $V$. dahliae has extensive host plant range (Smith, 1965). It causes Verticillium wilt in more than 200 plant species, including woody but more commonly dicotyledonous species of herbaceous annuals, perennials. Many of these host plants are cultivated and economically important crops like chili pepper, cotton, eggplant, lettuce, mint, potato, strawberry, tomato, and watermelon (Bhat and Subbarao, 1999; Papaioannou et al., 2013).

The control of Verticillium disease is difficult because they colonize the vascular system of the plants, they stay hidden inside the plant for most of their life cycle, and they produce microsclerotia which are durable for many years in the soil (Heale and Karapapa, 1999). Moreover there is no effective chemical control of Verticillium disease applicable in the field until now. The yield loss is correlated to the concentration of the microsclerotia in the soil (Wheeler et al., 1992). For this reason several studies focused in the control of Vertcillium diseases, especially $V$. dahliae; on the reduction of its soil borne inoculum (microsclerotia) (Nagtzaam et al., 1998; Subbarao et al., 1999; Tenuta and Lazarovits, 2002). Biological control using bacteria and plant extracts is one of the strategies in controlling Verticillium diseases (Uppal et al., 2008), other studies suggested solarization of the soil to increase the 
efficiency of the chemical which are used to control the microscerotia.(Ben-Yephet et al., 1988). Crop rotation (Xiao et al., 1998) and the use of Verticillum resistant cultivars are among the best strategies to reduce crop devastation through the disease (Johnson et al., 2013; Obermeier et al., 2013).

\section{Verticillium longisporum}

Verticillium longisporum was first isolated from horseradish in northern Germany in 1961 by Stark who believed that it was a sub-species of $V$. dahliae. He named it Verticillium dahliae var. longisporum, using the word "longisporum" after observing that the fungus has longer conidia than V. dahliae. In 1997 (Karapapa et al., 1997). differentiated Verticillium longisporum from $V$. dahliae on the basis of the morphological and molecular characterization of Verticillium longisporum in addition to the host specificity of the pathogen. They suggested that the fungus may have evolved by parasexual hybridization between a strain of $V$. alboatrum and a strain of $V$. dahliae, explaining its near diploid status (Karapapa et al., 1997). Later studies showed clear morphological and physiological differentiation between the two pathogens $V$. longisporum and $V$. dahliae (Zeise and Tiedemann, 2001). Using phylogenetic analysis it was confirmed that $V$. longisporum is diploid and a hybrid evolved through four different lineages and from three different parental species including $V$. dahliae (Inderbitzin et al., 2011). Despite its relation to $V$. dahliae, $V$. longisporum is pathogenically restricted to host plants from the Brassicaceae family, while $V$. dahliae is not able to cause disease on cruciferous plants (Zeise and Von Tiedemann, 2002).

Verticillium longisporum is the causal agent of Verticillium disease on oilseed rape (Brassica napus) (Karapapa et al., 1997; Steventon et al., 2002). In the UK it was first recorded in 2011 (Gladders et al., 2011). In Japan, V. longisporum is considered to be the major pathogen on Chinese cabbage (Ikeda et al., 2012). In Northern Germany, V. longisporum is one of the most important soil borne pathogens which may even benefit from rising temperatures due to global warming (Siebold and Tiedemann, 2012). In Sweden it is the dominant one among Verticillium species in soil (Johansson et al., 2006). 
The life cycle of Verticillium longisporum can be divided into three distinctive vegetative stages. In the dormant stage the pathogen can survive for many years in the soil by forming thick walled microsclerotia which serve as the inoculum and are triggered to germinate in the soil by root exudates of the host plants (Huisman, 1982; Mol and Riessen, 1995). The parasitic stage starts when the fungus enters the plants through the epidermal cells of the root cortex, colonizes the vascular system and remains restricted to it for most of its biotrophic part of its life cycle (Eynck et al., 2007; Zhou et al., 2006). Finally the fungus ends its life cycle in the saprophytic stage. Here, the symptoms of the disease are distinguishable and the fungus forms microsclerotia as resting structures and inoculum for the next life cycle. Fig. 2 shows the stages of the disease cycle of Verticillium spp. (Berlanger, and Powelson, n.d.) which was modified to show different stages of $V$. longisporum life cycle.

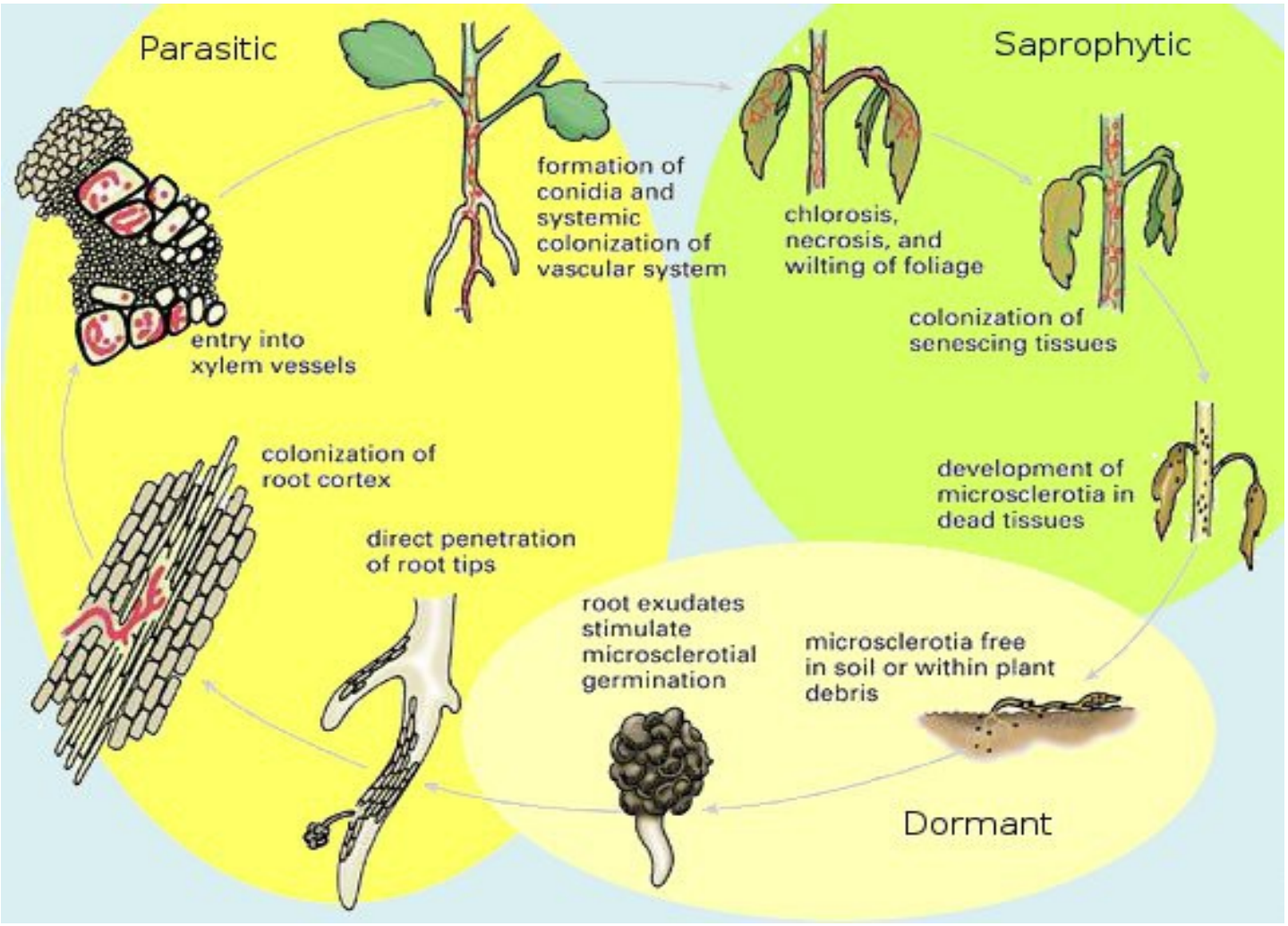

Figure 2. The life cycle of Verticillium spp.

(Modified after: Vickie Brewster, colored by Jesse Ewing), The original source:

http://www.apsnet.org/edcenter/intropp/lessons/fungi/ascomycetes/Pages/VerticilliumWilt.aspx 
In contrast to $V$. dahliae, however, $V$. longisporum does not cause wilt in the infected plants. Typical symptoms of infection occur in the shoot, these include stunted growth, mild chlorosis, necrotic lesion on the leaves, and premature senescence which can lead to losses in crop yield in susceptible cultivars.

Fig. 3 shows the symptoms of the infection of $V$. longisporum by root dipping inoculation on rapid cycling oilseed rape (Brassica napus) plants grown in the greenhouse.
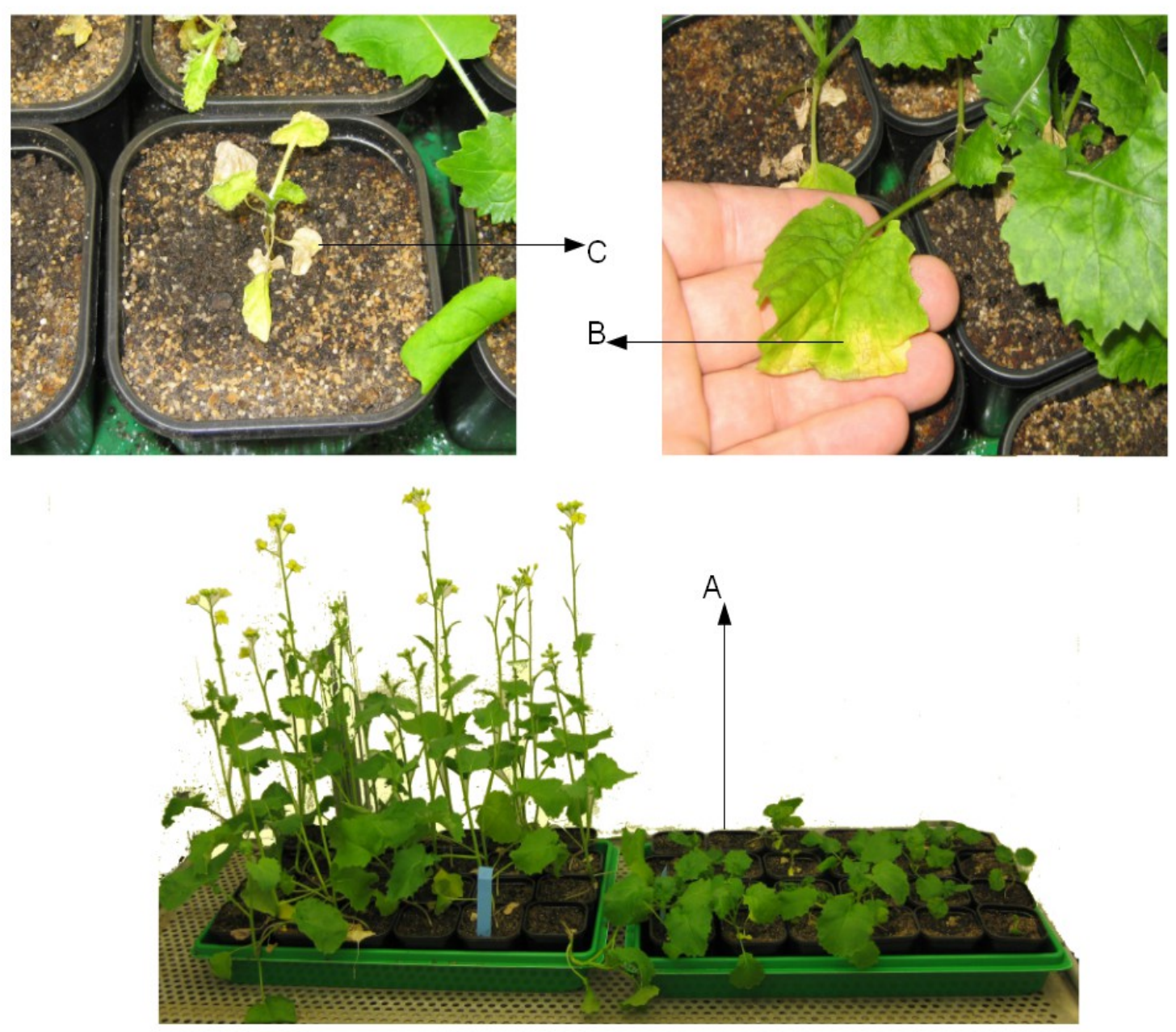

Figure 3. The typical symptoms caused by $V$. longisporum on oilseed rape in the greenhouse after root dip inoculation, A: stunting of the infected plants, B: chloroses on the leaves, C: necrotic lesion on the leaves. 


\section{Interactions between $V$. longisporum and $B$. napus}

$V$. longisporum enters the plant and stays restricted to the vascular tissues and specifically in the xylem vessels where the biochemical interactions take place (Ratzinger, 2008). The pathogen utilizes the amino acids and nutrition in the xylem sap, but the use of these nutrients has no limiting effect on the host and the resulting disease symptoms are not caused by the deprivation of these nutrients, and not by limiting water supplies or photosynthesis (Floerl et al., 2008) but rather the biochemical relation between the pathogen and the host plant (Kamble et al., 2012; Ratzinger, 2008).

Changes in the chemical composition of xylem sap of $B$. napus can be observed after infection with $V$. longisporum. For example, the phytohormone salicylic acid (SA) and its glucoside (SAG) showed increased concentrations after infection with the pathogen and their concentrations correlated with disease severity and the amount of the fungal DNA in the hypocotyl, while the other phytohormones jasmonic acid (JA) and abscisic acid (ABA) levels in xylem sap did not change after the infection (Ratzinger et al., 2009). However, this increased levels are not related to the resistance of the disease in B. napus (Kamble et al., 2012), whereas other phytohormones like ethylene seem to mediate $B$. napus response to $V$. longisporum infection (Floerl et al., 2008). A drastic increase of activity of phenylalanine ammonia lyase $(P A L)$ is correlated to the prevention of growth retardation indicating that the phenylpropanoid pathway plays the key role in disease resistance (Kamble et al., 2012). HPLC analysis of the hypocotyl of resistant double haploid populations of $B$. napus showed that there is correlation between the concentrations of a number of phenylpropanoids with $V$. longisporum resistance (Obermeier et al., 2013). Changes within the vascular system were also observed after infection with $V$. longisporum, such as accumulation of phenolics bound to cell walls and lignin and lignin-like modification (Eynck et al., 2009). By comparative metabolic profiling of xylem sap from $V$. longisporum infected and healthy $B$. napus plants, 19 signal changes were identified after infection, including groups of signals with increased or decreased intensities as well as groups of signals specific for infected but not found in healthy plants or V. dahliae infected B. napus plants (Ratzinger, 2008). In addition to small molecules, xylem sap of $B$. napus contains various proteins functioning as enzymes induced by the 
infection and they are involved in the defense reaction. The most well-known ones are chitinases (class I, IV), the disease resistance response protein (PR-1) and germin-like proteins (subfamily 1). Furthermore, xylem sap contains different peroxidases (Kehr et al., 2005). Brassica napus responds to $V$. longisporum infection by activating the defense mechanism in the apoplast which is based on defensive enzymes including endochitinase, peroxidase, PR4, and $\beta$-1,3-glucanase (Floerl et al., 2008). Macromolecules present in the xylem sap of $B$. napus are able to inhibit the fungal growth of $V$. longisporum (Singh et al., 2012).

In turn, $V$. longisporum is expected to produce phytotoxins to facilitate host plant invasion. Several previous studies, suggest that pathogenic Verticillium spp. produce a number of phytotoxins and elicitor compounds which can cause pathogen-specific symptoms in distant plant parts even in the absence of the fungus (Buchner et al., 1989; Nachmias et al., 1982). It is known that $V$. dahliae produces Vd-NEP protein which causes the wilt and elicit the biosynthesis of phytoalexins in cotton (Wang et al., 2004). A recent study identified secondary metabolites produced by Verticillium species which possess anti fungal activities including inhibition against Verticillium itself (You et al., 2009).

Beinhoff (2011) identified genes encoding for NEP protein biosynthesis in V. longisporum. He suggested that the protein plays a role as virulence factor as it is secreted into the xylem causing nutrition leakage of the adjacent tissues in favor of the fungus.

$V$. longisporum protects itself from oxidative stress especially in the saprophytic phase of its life cycle by producing six proteins. The most important one, catalase peroxidase, enables the fungus to perform the late phase of the disease (Singh et al., 2012). It is very likely that $V$. longisporum protects itself from $B$. napus phytoalexins by producing enzymes to detoxify them, in a similar mechanisms of other phytopathogenic fungi which infect $B$. napus (Pedras and Ahiahonu, 2005). 


\section{Secondary metabolites}

Secondary metabolites are a considerably wide class of organic compounds produced by living organisms including plants, fungi and bacteria. In contrast to the primary metabolites, they do not participate directly in the growth and development of the organism, so the absence of these compounds will not cause the immediate death of the organism.

For many decades, the chemistry of natural products focused on the discovery and structure elucidation of new natural products which are important pharmaceuticals in the field of drug development. This area has been extended recently due to an increasing interest in disclosing the ecological properties of many of these unknown secondary metabolites, especially to those showing an important ecological role in the interactions between the producing organisms and their environment (Demain and Fang, 2000; Kutchan, 2001). In this study, two groups of secondary metabolites will be involved, phytoalexins which are a large group of plant secondary metabolites, where the biosynthesis is elicited by microbial infection, and polyketides which are a large group of fungal secondary metabolites, encoded in fungi by polyketides synthase genes (PKS).

\section{Plant secondary metabolites}

Plants naturally produce a wide range of secondary metabolites with noticeably complex structures, a large number of these natural products are important pharmaceuticals. Both wild and cultivated plants are used as a source for discovering and isolating novel secondary metabolites because synthesizing them chemically is usually uneconomic (OksmanCaldentey and Inzé, 2004). Last decades have witnessed an increasing number of studies dealing with plant secondary metabolites, this enabled the development of a data base of known plant secondary metabolites including their chemical structure, function and producing organisms (Nakamura et al., 2013). Specific groups of plant secondary metabolites are conserved among plant families which make the chemical character of plants as important as morphological markers in plant taxonomy (Wink, 2003). 
Secondary metabolites produced by plants are important for plants in their interactions with the surrounding environment. Phytochemicals were naturally selected through millions of years of evolution, they play an important role in the survival and the reproductive fitness of the plants (Wink, 2003). Plants use them as chemical signals to attract animals for pollination or seed dispersing, or they are used in the defense mechanism of the plants against microorganisms (including fungi and bacteria), herbivore insects, and against other competing plants (Kliebenstein, 2013; Kutchan, 2001). Furthermore, many of plant secondary metabolites can act like phytohormones which have an important ecological role as signals used internally by plant to promote resistance within the plant itself or to activate resistance genes in neighboring plants (Shulaev et al., 1997; Sudha and Ravishankar, 2002).

\section{Phytoalexins}

Phytoalexins comprise a large class of secondary metabolites produced by higher plants in a part of their defense mechanism against microbial attack including fungal and bacterial infection (Darvill and Albersheim, 1984). These antimicrobial low molecular weight secondary metabolites are considered plant antibiotics (VanEtten et al., 1994). They were first described by Mueller and Buerger in 1940 as plant antibiotics produced by the plant upon microbial attack, their synthesis requires microbial infection as an elicitor. In contrast, phytoanticipins, another class of secondary metabolites also produced by plants, are present in plants before microbial infection occurs or they are produced after infection from preexisting components. Therefore some phytoalexins are considered phytoanticipins in other plants or even in other parts of the plant (Pedras and Yaya, 2010; VanEtten et al., 1994). Hence, the difference between these two classes is based on how they are synthesized rather than on their chemical structure. The role of phytoalexins is not restricted to plant pathogens but also affect herbivores (Hart et al., 1983). Furthermore they have an effect on the behavior of some insect which are affiliated with specific species of plants (Baur et al., 1998). There is an increasing interest in discovering phytoalexins and their synthetic pathways in plants to improve the resistance of cultivated crops toward diseases, which is an important biotechnological tool in sustainable agriculture.(Pedras and Yaya, 2013). Despite the widely varying chemical structure of phytoalexins within specific plant families, plants tend to synthesize them using 
the same or closely related building blocks (Pedras and Yaya, 2010). Plants from Brassicaceae are the source of important known phytoalexins (Pedras and Yaya, 2010; Pedras et al., 2000). Cruciferous plants respond to different types of biotic (including microbial infection by pathogenic or saprophytic microbes) or abiotic stress (like short irradiation intervals of high intensity, UV-light, exposure to heavy metals, salt solutions or a solution of particular chemicals) by accumulating blends of phytoalexins. The blend composition and the amount of their phytoalexins vary widely in same plants depending on the type of stress which elicits the synthesis of these phytoalexins (Pedras and Yaya, 2010).

For the detection of phytoalexins, HPLC-MS combined with a diode array detector (DAD) is considered the most effective and reliable method, whereas ${ }^{1} \mathrm{H}$ NMR spectra of such extracts and thin layer chromatography (TLC) are considered not to be reliable methods due to the high complexity of plant matrix extract and the low quantity of phytoalexins which can hardly be detected amongst high complex of compounds (Pedras and Yaya, 2010; Pedras et al., 2006). In order to elucidate the structure and to understand the bioactivity of phytoalexins, it is necessary to isolate and purify them. There are many factors which make the isolation and purification of these phytoalexins a difficult task. On the one hand, phytoalexins are produced in tiny amounts by plants and only in damaged tissues. On the other hand, extraction of phytoalexins from plant tissues is complex due to the production of other metabolites following the plant injury. Furthermore, the limited stability of phytoalexins under different extraction conditions and the purification procedure often requiring several chromatographic processes result in very small yields of the purified compounds (Pedras and Yaya, 2010; Pedras et al., 2006). So far, it was possible to isolate 44 phytoalexins from plants of the Brassicaceae family including wild species and cultivated crops. The chemical characteriziation of these isolated compounds was conducted by means of Nuclear Magnetic Resonance Spectroscopy (NMR), Infrared Spectroscopy (IR), and Ultraviolet-Visible Spectroscopy (UV) spectra combined with Mass Spectrometry (MS). Compounds were confirmed by the chemical synthesis of these new compounds and by their bio-activity against microbes (Pedras and Yaya, 2010; Pedras et al., 2011). 
These phytoalexins reported include important cruciferous phytoalexins like camalaxin, brassinin and cyclobrassinin (Pedras and Yaya, 2010; Pedras et al., 2011). Some of these isolated cruciferous phytoalexins were found to be produced naturally as phytoanticipins in some other Brassicacae species (Pedras and Zheng, 2010;Pedras et al., 2008a, 2008b). Time course of accumulation of cruciferous phytoalexins depends on the type of elicitor (Pedras et al., 2008a).

Phytoalexins from $B$. napus were previously investigated. In response to the infection with Plasmodiophora brassicae, a phytopathogen causing clubroot in several cruciferous crops, $B$. napus produces blends of phytoalexins detected in roots including spirobrassinin, 1methoxybrassinin, brassilexin, brassicanate $A$, sinalbin $B$, brassicanal $A$, 4-methoxybrassinin, 1-methoxybrassinin, 4-methoxydehydrocyclobrassinin, dehydrocyclobrassinin, caulilexin $A$, brassinin and cyclobrassinin, the latter being the most abundant phytoalexin in their blends. Fig. 4 shows the chemical structure of brassinin and cyclobrassinin. However, the effect of these phytoalexins on the pathogen $P$. brassicae remained unclear due to the difficulty of pathogen cultivation in vitro (Pedras et al., 2008b). The same study suggested that regulating phytoalexin and phytoanticipin synthesis may be subjected to a time shift between the early and late phase of infection (Pedras et al., 2008b). 

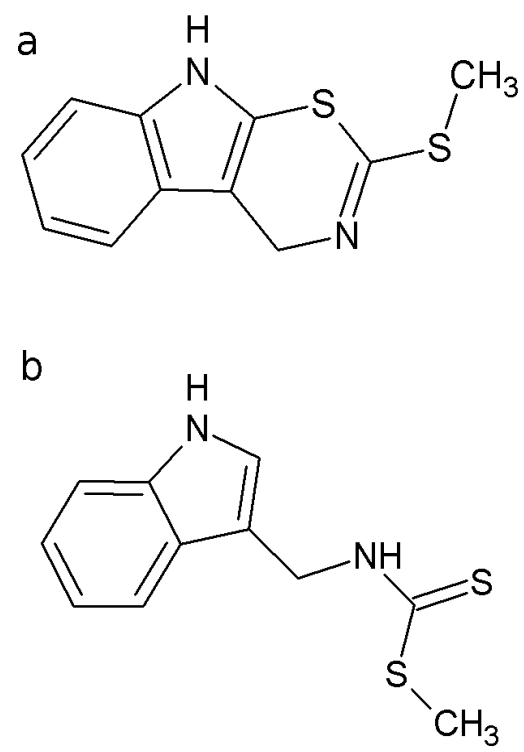

Figure 4. Chemical structure of cyclobrassinin (a) and brassinin (b)

Some pathogenic fungi effectively detoxify cruciferous phytoalexins and phytoanticipins, which seems to be a decisive factor for susceptibility of these cruciferous plants to diseases caused by these pathogens (Pedras and Ahiahonu, 2005; Pedras et al., 2009). This ability to detoxify phytoalexins is attributed to the possession of detoxifying enzymes. Brassinin detoxyfing enzymes were identified and isolated from different pathogenic fungi, (M. S. C. Pedras et al., 2008). For example, the soil-borne S. sclerotiorum is able to detoxify cyclobrassinin and brassilexin (Pedras and Hossain, 2006). Chemical synthesis of cruciferous phytoalexin is possible with high yield efficiency (Pedras et al., 2007), which can help in studying the bio-activity of these compounds besides their antimicrobial activities, for example, anti oxidative or anticancer properties (Pedras and Yaya, 2010; Romagnolo et al., 2012).

\section{Fungal secondary metabolites}

Microorganisms including filamentous fungi use distinctive biochemical pathways to produce numerous bioactive compounds characterized by low molecular weight. These secondary metabolites have evolved over millions of years as signals of chemical communication 
amongst these microorganisms or to inhibit the growth of other competitor organisms. (Rohlfs and Churchill, 2011). Despite many of these compounds were identified and characterized, most of them are still unknown. Many of the bioactive identified secondary metabolites are considered highly dangerous toxins like aflatoxins, some others are considered useful pharmaceuticals, for instance penicillin, while others are both toxic and useful at the same time like some Alkaloids (Keller et al., 2005). Many secondary metabolites produced by fungi and bacteria possess antimicrobial activities and are used against their competitors in the ecological niches. Necrotrophic phytopathogens synthesize lethal toxins and release them into plant tissues prior to invasion, while biotrophic phytopathogens are obliged to live on the living cells of the host plant and are usually unable to produce toxins (De Wit, 2013). Fungal secondary metabolites can be classified into polyketides, non ribosomal peptides, terpenes and indole alkaloids (Keller et al., 2005).

\section{Polyketides}

Polyketides cover an extensive array of secondary and primary metabolites produced by different macro- and microorganisms, including, but not limited to plants, animals, insects, bacteria and fungi (O'Hagan, 1995). These compounds are synthesized in prokaryotic and eukaryotic organisms in a mechanism similar to fatty acid synthesis (Hopwood and Sherman, 1990). Their synthesis in different organisms is carried out by three types of multifunctional enzymes called polyketide synthases (PKSs) (Keller et al., 2005). In fungi, polyketides are the most investigated class of fungal secondary metabolites with a wide range of chemical structures and functions. Some of the fungal polyketides are well characterized mycotoxins like the highly carcinogenic aflatoxin B1 (Watanabe et al., 1996) and Zearalenone which possesses oestrogenic activity in mammalians (Kim et al., 2005). Polyketides are also used by fungi as pigments, for example naphthopyrone compound YWA1 in Aspergillus nidulans (Watanabe et al., 1999). Since the discovery of the first polyketide encoding gene in fungi (Beck et al., 1990), there has been an increasing interest in fungal polyketides and the genes encoding for polyketide synthase, which can be utilized in the discovery of novel natural products (Hutchinson and Fujii, 1995; Lin et al., 2010). 
In Verticillium longisporum, a gene encoding for polyketide synthase was identified and named VI-PKS-1. The gene possesses high similarity to the previously identified PKS genes of the wA-type; a subclass of polyketide synthase genes in fungi, which are known to play a role in pigment synthesis pathways (Bingle et al., 1999), but not in the virulence or pathogenicity of $V$. longisporum towards $B$. napus (Beinhoff, 2011). After RNAi silencing of this gene, changes in the morphological and physiological levels were observed including increased growth rates or late pigmentation. Furthermore, a reddish color was observed in the PDA and PDB media, when V. longisporum VL43 was grown in it. No similar changes of the media color were observed, when VI-PKS-1 silenced type was grown (Beinhoff, 2011).

\section{Aim of the work}

The work presented in this thesis was conducted in the mycotoxin and molecular phytopathology unit. Department of Crop Sciences, University of Göttingen, under supervision of Prof. Dr. Petr Karlovsky. The author received a scholarship from the German Academic Exchange Service (DAAD) for doctorate studies in Germany. The thesis is released in the frame of International Ph.D. Program for Agricultural Sciences (IPAG) at the Faculty of Agricultural Sciences, Georg-August University of Göttingen. Parts of the work were conducted in collaboration with the department of plant biochemistry of the Albrecht-vonHaller Institute for Plant Sciences; headed by Prof. Dr. Ivo Feussner; at the University of Göttingen

The Aim of the work was:

- Isolation and purification of secondary metabolites from xylem sap of oilseed rape Brassica napus, which are specific for Verticillium longisporum infection (Chapter 2).

- Development of an assay to examine the inhibitory impact of $V$. longisporum infection against microorganisms, when they grow in the xylem sap of B. napus (Chapter 3 ).

- Observe changes in the metabolic profile of V. longisporum, when VI-PKS-1, a gene encoding for polyketide synthase in V. longisporum; is RNAi silenced (Chapter 4). 


\section{References}

Amtmann, A., 2009. Learning from Evolution: Thellungiella Generates New Knowledge on Essential and Critical Components of Abiotic Stress Tolerance in Plants. Mol. Plant 2, 3-12.

Aubé, C., Sackston, W.E., 1964. Verticillium wilt of forage legumes in Canada. Can. J. Plant Sci. 44, 427-432.

Barbara, D.J., Clewes, E., 2003. Plant pathogenic Verticillium species: how many of them are there? Mol. Plant Pathol. 4, 297-305.

Baur, R., Städler, E., Monde, K., Takasugi, M., 1998. Phytoalexins from Brassica (Cruciferae) as oviposition stimulants for the cabbage root fly, Delia radicum. Chemoecology 8, 163-168.

Beck, J., Ripka, S., Siegner, A., Schiltz, E., Schweizer, E., 1990. The multifunctional 6methylsalicylic acid synthase gene of Penicillium patulum. Eur. J. Biochem. 192, 487-498.

Beinhoff, M., 2011. Molecular and functional characterization of potential pathogenicity related genes from Verticillium longisporum. Georg-August-Universität Göttingen, Göttingen, Germany.

Ben-Yephet, Y., Melero-Vera, J.M., DeVay, J.E., 1988. Interaction of soil solarization and metham-sodium in the destruction of Verticillium dahliae and Fusarium oxysporum $f$. sp. vasinfectum. Crop Prot. 7, 327-331.

Berlanger, I., Powelson, M.L., 2000 Verticillium wilt, The Plant Health Instructor. Oregon State University, USA.

Bingle, L.E., Simpson, T.J., Lazarus, C.M.,1999. Ketosynthase domain probes identify two subclasses of fungal polyketide synthase genes. Fungal Genet. Biol. 26, 209-223.

Bhat, R.G., Subbarao, K.V., 1999. Host Range Specificity in Verticillium dahliae. Phytopathology 89, 1218-1225. 
Buchner, V., Burstein, Y., Nachmias, A., 1989. Comparison of Verticillium dahliae-produced phytotoxic peptides purified from culture fluids and infected potato stems. Physiol. Mol. Plant Pathol. 35, 253-269.

Darvill, A.G., Albersheim, P., 1984. Phytoalexins and their Elicitors-A Defense Against Microbial Infection in Plants. Annu. Rev. Plant Physiol. 35, 243-275.

De Wit, P.J.G.M., 2013. Microbial toxins in the green world. Fems Microbiol. Rev. 37, 1-2.

Demain, A.L., Fang, A., 2000. The Natural Functions of Secondary Metabolites, in: Fiechter, P.D.A. (Ed.), History of Modern Biotechnology I, Advances in Biochemical Engineering/Biotechnology. Springer Berlin Heidelberg, pp. 1-39.

Diepenbrock, W., 2000. Yield analysis of winter oilseed rape (Brassica napus L.): a review. Field Crops Res. 67, 35-49.

Dunker, S., Keunecke, H., Steinbach, P., Von Tiedemann, A., 2008. Impact of Verticillium longisporum on Yield and Morphology of Winter Oilseed Rape (Brassica napus) in Relation to Systemic Spread in the Plant. J. Phytopathol. 156, 698-707.

Eynck, C., Koopmann, B., Karlovsky, P., von Tiedemann, A., 2009. Internal Resistance in Winter Oilseed Rape Inhibits Systemic Spread of the Vascular Pathogen Verticillium longisporum. Phytopathology 99, 802-811.

Fahleson, J., Hu, Q., Dixelius, C., 2004. Phylogenetic analysis of Verticillium species based on nuclear and mitochondrial sequences. Arch. Microbiol. 181, 435-442.

FAO, 2013.The Statistics Division of the FAO, FAOSTAT, Food and Agriculture Organization of the United Nations.

Floerl, S., Druebert, C., Majcherczyk, A., Karlovsky, P., Kües, U., Polle, A., 2008. Defence reactions in the apoplastic proteome of oilseed rape (Brassica napus var. napus) attenuate Verticillium longisporum growth but not disease symptoms. Bmc Plant Biol. 8, 129.

Fradin, E.F., Thomma, B.P.H.J., 2006. Physiology and molecular aspects of Verticillium wilt diseases caused by V. dahliae and V. albo-atrum. Mol. Plant Pathol. 7, 71-86. 
Gladders, P., Smith, J.A., Kirkpatrick, L., Clewes, E., Grant, C., Barbara, D., Barnes, A.V., Lane, C.R., 2011. First record of Verticillium wilt (Verticillium longisporum) in winter oilseed rape in the UK. New Dis. Reports 23, Article 8.

Hart, S.V., Kogan, M., Paxton, J.D., 1983. Effect of soybean phytoalexins on the herbivorous insects mexican bean beetle and soybean looper. J. Chem. Ecol. 9, 657-672.

Heale, J.B., Karapapa, V.K., 1999. The verticillium threat to canada's major oilseed crop: canola. Can. J. Plant Pathol. 21, 1-7.

Hopwood, D.A., Sherman, D.H., 1990. Molecular Genetics of Polyketides and its Comparison to Fatty Acid Biosynthesis. Annu. Rev. Genet. 24, 37-62.

Hutchinson, C.R., Fujii, I., 1995. Polyketide Synthase Gene Manipulation: A StructureFunction Approach in Engineering Novel Antibiotics. Annu. Rev. Microbiol. 49, 201-238.

Ikeda, K., Banno, S., Watanabe, K., Fujinaga, M., Ogiso, H., Sakai, H., Tanaka, H., Miki, S., Shibata, S., Shiraishi, T., Fujimura, M., 2012. Association of Verticillium dahliae and Verticillium longisporum with Chinese cabbage yellows and their distribution in the main production areas of Japan. J. Gen. Plant Pathol. 78, 331-337.

Inderbitzin, P., Davis, R.M., Bostock, R.M., Subbarao, K.V., 2011. The Ascomycete Verticillium longisporum Is a Hybrid and a Plant Pathogen with an Expanded Host Range. Plos One 6, e18260.

Johansson, A., Goud, J.-K.C., Dixelius, C., 2006. Plant Host Range of Verticillium longisporum and Microsclerotia Density in Swedish Soils. Eur. J. Plant Pathol. 114, 139-149.

Johnson, D.A., Baker, R., Boydston, R.A., 2013. Field evaluation of mutant and hybrid lines of mint for resistance to Verticillium wilt and yield. Crop Prot. 43, 1-6.

Kamble, A., Koopmann, B., von Tiedemann, A., 2012. Induced resistance to Verticillium longisporum in Brassica napus by $\beta$-aminobutyric acid. Plant Pathol.62, 552-561. 
Karapapa, V.K., Bainbridge, B.W., Heale, J.B., 1997. Morphological and molecular characterization of Verticillium longisporum comb, nov., pathogenic to oilseed rape. Mycol. Res. 101, 1281-1294.

Katz, S.H., Weaver, W.W., 2003. Encyclopedia of food and culture. Scribner, New York.

Kehr, J., Buhtz, A., Giavalisco, P., 2005. Analysis of xylem sap proteins from Brassica napus. Bmc Plant Biol. 5, 11.

Keller, N.P., Turner, G., Bennett, J.W., 2005. Fungal secondary metabolism - from biochemistry to genomics. Nat. Rev. Microbiol. 3, 937-947.

Kim, Y.-T., Lee, Y.-R., Jin, J., Han, K.-H., Kim, H., Kim, J.-C., Lee, T., Yun, S.-H., Lee, Y.-W., 2005. Two different polyketide synthase genes are required for synthesis of zearalenone in Gibberella zeae. Mol. Microbiol. 58, 1102-1113.

Kliebenstein, D.J., 2013. Making new molecules-evolution of structures for novel metabolites in plants. Curr. Opin. Plant Biol.16, 112-117

Kutchan, T.M., 2001. Ecological Arsenal and Developmental Dispatcher. The Paradigm of Secondary Metabolism. Plant Physiol. 125, 58-60.

Lin, X., Huang, Y.J., Zheng, Z.H., Su, W.J., Qian, X.M., Shen, Y.M., 2010. Endophytes from the pharmaceutical plant, Annona squamosa: isolation, bioactivity, identification and diversity of its polyketide synthase gene. Fungal Divers. 41, 41-51.

Mol, L., Riessen, H.W. van, 1995. Effect of plant roots on the germination of microsclerotia of Verticillum dahliae. Eur. J. Plant Pathol. 101, 673-678.

Nachmias, A., Buchner, V., Krikun, J., 1982. Comparison of protein-lipopolysaccharide complexes produced by pathogenic and non-pathogenic strains of Verticillium dahliae Kleb. from potato. Physiol. Plant Pathol. 20, 213-216,IN17,217-221. 
Nagtzaam, M.P.M., Bollen, G.J., Termorshuizen, A.J., 1998. Wirkung von Talaromyces flavus allein oder in Kombination mit anderen Antagonisten bei der Bekämpfung von Verticillium dahliae in Klimakammerversuchen. J. Phytopathol. 146, 165-173.

Nakamura, K., Shimura, N., Otabe, Y., Hirai-Morita, A., Nakamura, Y., Ono, N., Ul-Amin, M.A., Kanaya, S., 2013. KNApSAcK-3D: A Three-Dimensional Structure Database of Plant Metabolites. Plant Cell Physiol. 54, e4-e4.

Nazar, R.N., Hu, X., Schmidt, J., Culham, D., Robb, J., 1991. Potential use of PCRamplified ribosomal intergenic sequences in the detection and differentiation of Verticillium wilt pathogens. Physiol. Mol. Plant Pathol. 39, 1-11.

O’Hagan, D., 1995. Biosynthesis of fatty acid and polyketide metabolites. Nat. Prod. Rep. 12, 1.

Obermeier, C., Hossain, M.A., Snowdon, R., Knüfer, J., Tiedemann, A. von, Friedt, W., 2013. Genetic analysis of phenylpropanoid metabolites associated with resistance against Verticillium longisporum in Brassica napus. Mol. Breed. 1-15.

Oksman-Caldentey, K.-M., Inzé, D., 2004. Plant cell factories in the post-genomic era: new ways to produce designer secondary metabolites. Trends Plant Sci. 9, 433-440.

Papaioannou, I.A., Ligoxigakis, E.K., Vakalounakis, D.J., Markakis, E.A., Typas, M.A., 2013. Phytopathogenic, morphological, genetic and molecular characterization of a Verticillium dahliae population from Crete, Greece. Eur. J. Plant Pathol. 1-20.

Pedras, M.S.C., Adio, A.M., Suchy, M., Okinyo, D.P.O., Zheng, Q.-A., Jha, M., Sarwar, M.G., 2006. Detection, characterization and identification of crucifer phytoalexins using highperformance liquid chromatography with diode array detection and electrospray ionization mass spectrometry. J. Chromatogr. A 1133, 172-183.

Pedras, M.S.C., Ahiahonu, P.W.K., 2005. Metabolism and detoxification of phytoalexins and analogs by phytopathogenic fungi. Phytochemistry 66, 391-411. 
Pedras, M.S.C., Chumala, P.B., Jin, W., Islam, M.S., Hauck, D.W., 2009. The phytopathogenic fungus Alternaria brassicicola: Phytotoxin production and phytoalexin elicitation. Phytochemistry 70, 394-402.

Pedras, M.S.C., Hossain, M., 2006. Metabolism of crucifer phytoalexins in Sclerotinia sclerotiorum: detoxification of strongly antifungal compounds involves glucosylation. Org. Biomol. Chem. 4, 2581-2590.

Pedras, M.S.C., Minic, Z., Jha, M., 2008. Brassinin oxidase, a fungal detoxifying enzyme to overcome a plant defense - purification, characterization and inhibition. Febs J. 275, 36913705.

Pedras, M.S.C., Okanga, F.I., Zaharia, I.L., Khan, A.Q., 2000. Phytoalexins from crucifers: synthesis, biosynthesis, and biotransformation. Phytochemistry 53, 161-76.

Pedras, M.S.C., Yaya, E.E., 2010. Phytoalexins from Brassicaceae: News from the front. Phytochemistry $71,1191-1197$.

Pedras, M.S.C., Yaya, E.E., 2013. Dissecting metabolic puzzles through isotope feeding: a novel amino acid in the biosynthetic pathway of the cruciferous phytoalexins rapalexin $A$ and isocyalexin A. Org. Biomol. Chem. 11, 1149.

Pedras, M.S.C., Yaya, E.E., Glawischnig, E., 2011. The phytoalexins from cultivated and wild crucifers: chemistry and biology. Nat. Prod. Rep. 28, 1381-405.

Pedras, M.S.C., Zheng, Q.-A., 2010. Metabolic responses of Thellungiella halophila/salsuginea to biotic and abiotic stresses: Metabolite profiles and quantitative analyses. Phytochemistry $71,581-589$.

Pedras, M.S.C., Zheng, Q.-A., Gadagi, R.S., Rimmer, S.R., 2008a. Phytoalexins and polar metabolites from the oilseeds canola and rapeseed: Differential metabolic responses to the biotroph Albugo candida and to abiotic stress. Phytochemistry 69, 894-910.

Pedras, M.S.C., Zheng, Q.-A., Sarwar, M.G., 2007. Efficient synthesis of brussalexin A, a remarkable phytoalexin from Brussels sprouts. Org. Biomol. Chem. 5, 1167. 
Pedras, M.S.C., Zheng, Q.-A., Strelkov, S., 2008b. Metabolic Changes in Roots of the Oilseed Canola Infected with the Biotroph Plasmodiophora brassicae: Phytoalexins and Phytoanticipins. J. Agric. Food Chem. 56, 9949-9961.

Pegg, G.F., Brady, B.L., 2002. Verticillium wilts 2002 pp. UK.

Pérez-Artés, E., García-Pedrajas, M.D., Bejarano-Alcázar, J., Jiménez-Díaz, R.M., 2000. Differentiation of Cotton-defoliating and Nondefoliating Pathotypes of Verticillium dahliae by RAPD and Specific PCR Analyses. Eur. J. Plant Pathol. 106, 507-517.

Ratzinger, A., 2008. Development and application of LC-MS-based differential metabolic profiling in plant systems. Georg-August-Universität Göttingen, Göttingen, Germany.

Ratzinger, A., Riediger, N., von Tiedemann, A., Karlovsky, P., 2009. Salicylic acid and salicylic acid glucoside in xylem sap of Brassica napus infected with Verticillium longisporum. J. Plant Res. 122, 571-579.

Rohlfs, M., Churchill, A.C.L., 2011. Fungal secondary metabolites as modulators of interactions with insects and other arthropods. Fungal Genet. Biol. 48, 23-34.

Romagnolo, D.F., Davis, C.D., Milner, J.A., 2012. Phytoalexins in cancer prevention. Front. Biosci. J. Virtual Libr. 17, 2035-2058.

Shulaev, V., Silverman, P., Raskin, I., 1997. Airborne signalling by methyl salicylate in plant pathogen resistance. Nature 385, 718-721.

Siebold, M., Tiedemann, A. von, 2012. Potential effects of global warming on oilseed rape pathogens in Northern Germany. Fungal Ecol. 5, 62-72.

Singh, S., Braus-Stromeyer, S.A., Timpner, C., Valerius, O., von Tiedemann, A., Karlovsky, P., Druebert, C., Polle, A., Braus, G.H., 2012. The plant host Brassica napus induces in the pathogen Verticillium longisporum the expression of functional catalase peroxidase which is required for the late phase of disease. Mol. Plant-Microbe Interactions Mpmi 25, 569-581. 
Smith, H.C., 1965. The morphology of Verticillium albo-atrum, V. dahliae, and V. tricorpus. New Zealand J. Agric. Res. 8, 450-478.

Steventon, L.A., Fahleson, J., Hu, Q., Dixelius, C., 2002. Identification of the causal agent of Verticillium wilt of winter oilseed rape in Sweden, V. Iongisporum. Mycol. Res. 106, 570578.

Subbarao, K.V., Hubbard, J.C., Koike, S.T., 1999. Evaluation of Broccoli Residue Incorporation into Field Soil for Verticillium Wilt Control in Cauliflower. Plant Dis. 83, 124-129.

Sudha, G., Ravishankar, G.A., 2002. Involvement and interaction of various signaling compounds on the plant metabolic events during defense response, resistance to stress factors, formation of secondary metabolites and their molecular aspects. Plant Cell Tissue Organ Cult. 71, 181-212.

Tenuta, M., Lazarovits, G., 2002. Ammonia and Nitrous Acid from Nitrogenous Amendments Kill the Microsclerotia of Verticillium dahliae. Phytopathology 92, 255-264.

Uppal, A.K., El Hadrami, A., Adam, L.R., Tenuta, M., Daayf, F., 2008. Biological control of potato Verticillium wilt under controlled and field conditions using selected bacterial antagonists and plant extracts. Biol. Control 44, 90-100.

USDA, April2013. Major Oilseeds: World Supply and Distribution (Commodity View).

VanEtten, H., Mansfield, J., Bailey, J., Farmer, E., 1994. Two Classes of Plant Antibiotics: Phytoalexins versus "Phytoanticipins". Plant Cell 6, 1191-1192.

Wang, J.-Y., Cai, Y., Gou, J.-Y., Mao, Y.-B., Xu, Y.-H., Jiang, W.-H., Chen, X.-Y., 2004. VdNEP, an Elicitor from Verticillium dahliae, Induces Cotton Plant Wilting. Appl. Environ. Microbiol. 70, 4989-4995.

Watanabe, A., Fujii, I., Sankawa, U., Mayorga, M.E., Timberlake, W.E., Ebizuka, Y., 1999. Re-identification of Aspergillus nidulans wA gene to code for a polyketide synthase of naphthopyrone. Tetrahedron Lett. 40, 91-94. 
Watanabe, C.M.H., Wilson, D., Linz, J.E., Townsend, C.A., 1996. Demonstration of the catalytic roles and evidence for the physical association of type I fatty acid synthases and a polyketide synthase in the biosynthesis of aflatoxin B1. Chem. Biol. 3, 463-469.

Wheeler, T.A., Madden, L.V., Rowe, R.C., Riedel, R.M., 1992. Modeling of Yield Loss in Potato Early Dying Caused by Pratylenchus penetrans and Verticillium dahliae. J. Nematol. 24, 99-102.

Wink, M., 2003. Evolution of secondary metabolites from an ecological and molecular phylogenetic perspective. Phytochemistry 64, 3-19.

Xiao, C.L., Subbarao, K.V., Schulbach, K.F., Koike, S.T., 1998. Effects of Crop Rotation and Irrigation on Verticillium dahliae Microsclerotia in Soil and Wilt in Cauliflower. Phytopathology 88, 1046-1055.

You, F., Han, T., Wu, J., Huang, B., Qin, L., 2009. Antifungal secondary metabolites from endophytic Verticillium sp. Biochem. Syst. Ecol. 37, 162-165.

Zare, R., Gams, W., Starink-Willemse, M., Summerbell, R.C., 2007. Gibellulopsis, a suitable genus for Verticillium nigrescens, and Musicillium, a new genus for $V$. theobromae. Nova Hedwig. 85, 463-489.

Zeise, K., Tiedemann, A. von, 2001. Morphological and Physiological Differentiation among Vegetative Compatibility Groups of Verticillium dahliae in Relation to V. Iongisporum. J. Phytopathol. 149, 469-475.

Zeise, K., Von Tiedemann, A., 2002. Host Specialization among Vegetative Compatibility Groups of Verticillium dahliae in Relation to Verticillium longisporum. J. Phytopathol. 150, 112-119.

Zhou, L., Hu, Q., Johansson, A., Dixelius, C., 2006. Verticillium longisporum and V. dahliae: infection and disease in Brassica napus. Plant Pathol. 55, 137-144. 


\title{
Chapter 2: Isolation and purification of secondary metabolites from xylem sap of Brassica napus infected with Verticillium longisporum
}

\author{
Husam Ibrahem Aroud, Astrid Ratzinger and Petr Karlovsky \\ Molecular Phytopathology and Mycotoxin Research Unit, Department of Crop Sciences, University of \\ Göttingen, Grisebachstrasse 6, 37077 Göttingen, Germany
}

\section{Abstract}

Verticillium longisporum is a devastating vascular fungus which infects oilseed rape (Brassica napus.) The exchange of secondary metabolites produced from the pathogen and the host plant occur in the xylem vessels and specifically in the xylem sap. A previous study identified compounds in the xylem sap of $B$. napus which are specific to the infection of $V$. longisporum. In this research work we describe the isolation and the purification of one of these compounds which has the exact mass of $612.1197 \mathrm{Da}$. Xylem sap was collected from a large number of rapid cycling oilseed rape plants infected with $V$. longisporum. Macromolecules were removed from the xylem sap using filter membrane prior to the purification process. Purification was conducted by using preparative HPLC system upgraded from the analytical HPLC-MS that was previously used in the nontargeted metabolic profiling. Further purification was conducted by using a semi analytical UPLC system. The isolation of the compound was accomplished successfully but the yield of the purification was not sufficient for structure elucidation. The mass spectrometric analysis indicates that a compound of $\mathrm{m} / \mathrm{z} 527.1291[\mathrm{M}+\mathrm{H}]^{+}$is a result of fragmentation of the purified compound. We believe that the purified compound is produced by the plant as a part of its defense mechanism which is induced specifically by $V$. longisporum infection and therefore it is considered a phytoalexin.

Key words: Verticillium longisporum, Brassica napus, xylem sap, phytoalexins 


\section{Introduction}

Verticillium longisporum is a soil-borne vascular pathogenic fungus of the ascomycete Verticillium pathogens which cause vascular diseases to their host plants in temperate and sub-tropical areas (Pegg and Brady, 2002). Among Verticillium species, V. longisporum is specific to host plants of Brassicaceae family (Zeise and Von Tiedemann, 2002) and it causes Verticillium disease on oilseed rape (Brassica napus) (Karapapa et al., 1997). It is considered a serious potential threat to oilseed cultivation in different parts of the world (Heale and Karapapa, 1999). In Sweden, it is among the most common Verticillium pathogens (Johansson et al., 2006) and it causes losses in the yield of oilseed rape in Germany (Dunker et al., 2008). The life cycle of Verticillium longisporum can be divided into three distinct vegetative stages. In the dormant stage, the thick walled microsclerotia serve as potential inoculum and this survives for many years in soil. The fungus attacks the plants after the germination of the microsclerotia which are induced by root exudates of the host plants (Huisman, 1982; Mol and Riessen, 1995). The fungus enters the plants through the epidermal cells of the root cortex and quickly colonizes the vascular system and remains there for most of the biotrophic part of its life cycle (Eynck et al., 2007; Zhou et al., 2006). After causing the disease symptoms in the late stage of infection, the fungus ends its life cycle as a saprophytic fungus. In contrast to $V$. dahliae, V. longisporum does not cause wilt to the infected plants but typical symptoms of the infection occur in the late stage of infection including stunted growth, mild chlorosis and premature senescence of susceptible host plants, and severe infection can lead to substantial losses in crop yield (Dunker et al., 2008; Eynck et al., 2007; Floerl et al., 2008). The stunted growth of the infected plants is not caused by limitation of nutrition, the infection has also limited or no effect on the water supplies and photosynthesis in the infected plants (Floerl et al., 2008). The biochemical interactions between the pathogen and the infected host plant play the key role in development of the disease symptoms (Kamble et al., 2012; Ratzinger, 2008).

Plants have a natural immune system based on the production of secondary metabolites which form an effective defense mechanism in response to invading pathogenic bacteria and fungi (Clay, 2011). Secondary metabolites produced by plants have a very crucial role in resistance against the pathogen. Changes in the metabolic levels can be observed in $B$. 
napus after $V$. longisporum infection, for example, accumulation of soluble phenolics and lignin modification forms inside the vascular system (Eynck et al., 2009). Phenylpropanoid pathway plays a key role in the resistance of $B$. napus against $V$. longisporum (Kamble et al., 2012). Increased concentrations of salicylic acid (SA) and its glucoside (SAG) were detected after infection with $V$. longisporum. Their concentrations correlated with disease severity and the amount of the fungal DNA in the hypocotyl (Ratzinger et al., 2009). However (SA) is not related to disease resistance in B. napus (Kamble et al., 2012).

While fungal pathogens produce secondary metabolites that ease their attack against the host plants, plants in turn respond to different sorts of biotic and abiotic stresses by producing low molecular weight secondary metabolites. These compounds are called phytoalexins. In contrast to phytoanticipins, phytoalexins are bio-synthesized de novo only under stress and they are not bio-synthesized in healthy plants (Darvill and Albersheim, 1984; Pedras, 2010). Phytoalexins form an important part of plant biochemical and chemical defense mechanism against herbivores, microbial attack and other types of stress (Darvill and Albersheim, 1984; Hart et al., 1983; Pedras, 2010). In addition to their antimicrobial properties against many plant pathogens, preclinical evidence has shown that some of phytoalexins may possess anticancer properties (Romagnolo et al., 2012). It is known that these compounds are produced in a large number of plants including crucifers (Pedras, 2010; Pedras et al., 2000). Since the first phytoalexins were reported it has been the aim of many studies to discover new phytoalexins (Keen N. T. and Bruegger B., 1977). Phytoalexins are produced by plants in blends with various compositions depending on the elicitor and also on the plant species. Plants exploit common chemical building blocks in synthesizing a great variety of phytoalexins. Crucifers synthesize a broad range of phytoalexins with similar chemical structures but showing different types of biological activities including antimicrobial properties (M Pedras, 2010; Pedras et al., 2000). Increased concentrations of phytoalexins like rapalxin have been observed in Brassica species as a response to pathogens (Pedras, 2011).

In order to have full understanding of the bioactivities of phytoalexins, isolation and purification of these secondary metabolites is required to elucidate their chemical structure, but it is not uncomplicated task due to the complexity of the extraction of plant tissues and the very tiny amount of the resulting final product. Furthermore, the stability of 
the purified phytoalexins is often a very critical factor in this process (Pedras, 2011, 2010; Pedras et al., 2006).

It was possible to chemically characterize 44 phytoalexins in Brassica napus by using mean of HPLC combined with the analysis of NMR spectroscopic data for structure elucidation of these phytoalexins (Pedras, 2010). In previous PhD research work, (Ratzinger, 2008) conducted non-targeting metabolic profiling of the xylem sap of $V$. longisporum and $V$. dahliae infected rapid cycling oilseed rape (Brassica napus), and compared them with healthy plants. Eight compounds were identified in the xylem sap of $B$. napus which are specific to the infection of $V$. longisporum. Tandem mass spectrometry (MS-MS) revealed that all these compounds possess common chemical building blocks derived from indole (Ratzinger, 2008). This is not an uncommon character of cruciferous phytoalexins (Pedras et al., 2006). The compound of mass to charge of $\mathrm{m} / \mathrm{z} 613[\mathrm{M}+\mathrm{H}]^{+}$ does not match any masses of the previously known phytoalexins from Brassica napus (Ratzinger, 2008).

\section{Aim of the work}

The aim of this study was to isolate and purify the most abundant compound found to be specific for $V$. longisporum infection in xylem sap of B.napus. This compound was detected in positive and negative modes of ionization with $m / z 613[\mathrm{M}+\mathrm{H}]^{+}$, and $m / z 611[\mathrm{M}-\mathrm{H}]$, respectively. Another goal of the study was to establish a standard purification procedure for secondary metabolites from xylem sap which can be applied to other infection specific compounds in xylem sap of $B$. napus. 


\section{Authors' contributions}

Husam Ibrahem Aroud carried out inoculation experiments, xylem sap extraction and metabolites purification and preliminary characterization by HPLC-MS and wrote most of the manuscript. Kirstin Feussner and Cornelia Herrfurth (Department of Plant Biochemistry of the Albrecht-von-Haller Institute for Plant Sciences, University of Göttingen) carried out the second step of purification and characterized purified metabolites using HPLC with high-resolution MS detection, recorded their UV absorption spectra and wrote the corresponding part of the Material and Methods section. Astrid Ratzinger established the methods for HPLC-MS and carried out differential metabolic profiling of xylem sap from infected and healthy plants. Petr Karlovsky designed and supervised the study and corrected the manuscript.

\section{Materials and Methods}

The analytical method of HPLC-MS used in this chapter to examine the eluted fractions from the preparative HPLC column was established and described by Astrid Ratzinger in her PhD thesis thesis (Ratzinger, 2008). The same method was scaled-up to a preparative method in order to maintain the same conditions of the experiment.

\section{Fungal isolate of $V$. longisporum}

The pathogenic fungus Verticillium longisporum strain VL 43 which was isolated from Brassica napus plants grown in northern Germany as described in (Zeise and Tiedemann, 2001); was used to infect $B$. napus plants. Fungal stocks with $1 \times 10^{6}$ spore $/ \mathrm{ml}$ were stored at $-80^{\circ} \mathrm{C}$ in $25 \%$ glycerol. These fungal stock was used to prepare $V$. longiporum inocula. 


\section{Brassica napus plants}

Xylem sap was collected from large number of infected rapid cycling oilseed rape plants (Brassica napus var. napus, genome ACaacc), the seeds were provided by Paul $\mathrm{H}$. Williams (Department of Plant Biology, University of Wisconsin-Madison, Crucifer Genetics Cooperative, Stock number 5). Before sowing them to grow, seeds were subjected to surface sterilization by dipping them in $70 \%$ ethanol for 30 seconds, followed by rinsing them with tab water three times for 30 seconds. The seeds were sown in sterilized silica sand and left to germinate under sterile controlled condition of $22 / 20^{\circ} \mathrm{C}$ day/ night), $15 \mathrm{~h}$ day long and $60 \%$ humidity, with regular irrigation. The short life cycle of rapid cycling oilseed plants facilitated collect a large amount of xylem sap.

\section{Preparation of Verticillium longisporum inoculum}

$500 \mu \mathrm{l}$ of aforementioned Verticillium longisporum stock with $\left(1 \times 10^{6}\right.$ spore $\left./ \mathrm{ml}\right)$ were added to $300 \mathrm{ml}$ Czapek Dox broth. The inoculated broth was incubated at $23{ }^{\circ} \mathrm{C}$ on a rotary shaker at $100 \mathrm{rpm}$ and kept in the dark. A sterile sieve was used after 10-14 days to filter the fungal mycelia. Spore concentration was determined by counting spores using a haemocytometer (Thoma) and then diluted to $1 \times 10^{6} \mathrm{spore} / \mathrm{ml}$.

\section{Inoculation of the seedlings with Verticillium longisporum}

Seven to ten day-old seedlings were inoculated by root-dipping for 30-40 minutes in a spore suspension of $1 \times 10^{6}$ spores/ml of $V$. longisporum isolate VL43. After inoculation, single seedlings were transferred to pots with a sterile sand-soil mixture (1:1) and grown under the controlled conditions as mentioned above. Fig. 1 shows the plants transferred to single pots and growing them in climatic chamber. 


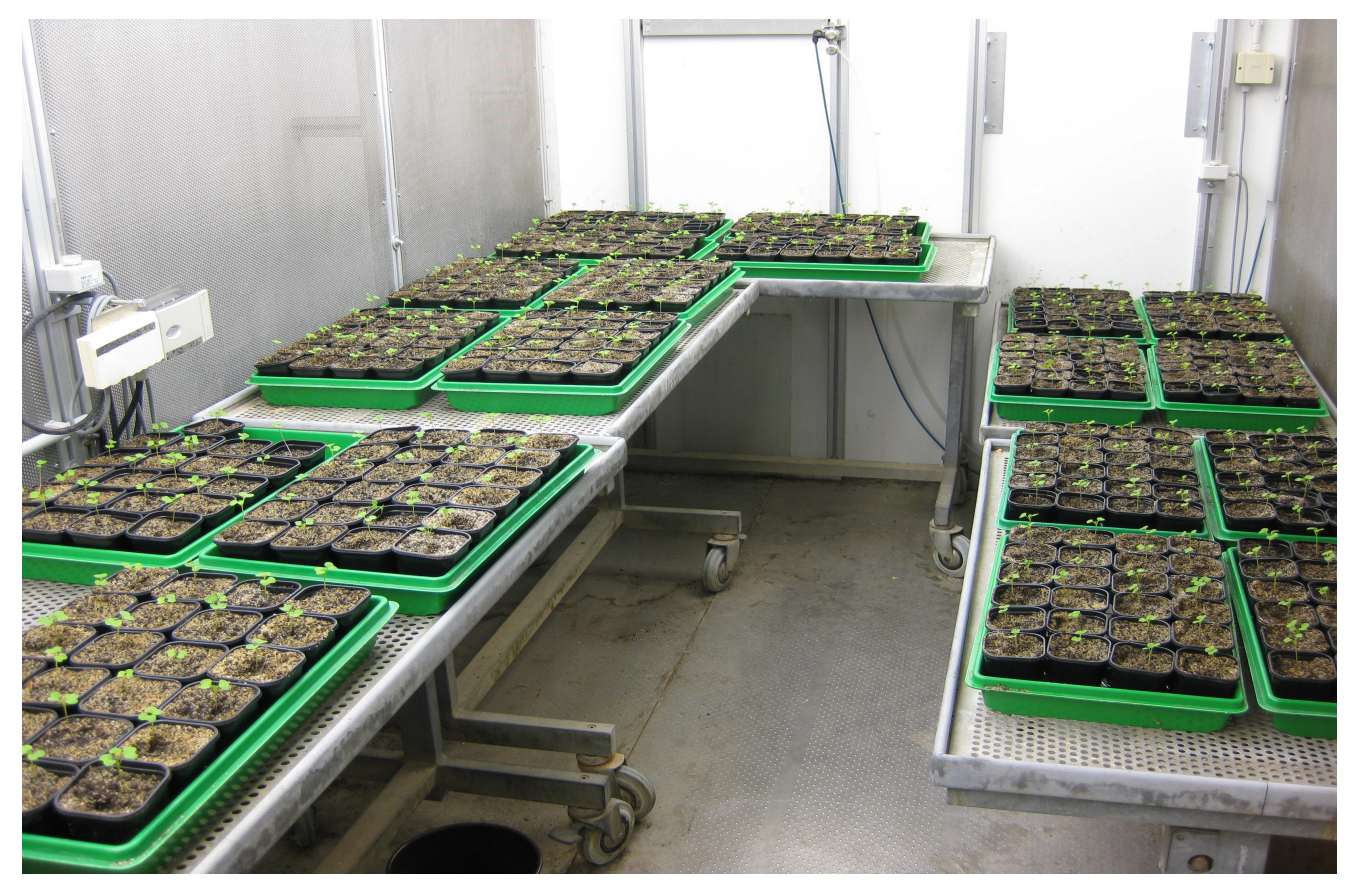

Figure 1. Inoculation of the seedlings of $B$. napus.

Large numbers of $B$. napus plants were inoculated with spore suspension of $V$. longisporum and then each single seedling were transferred to a pot.

\section{Xylem sap collection}

The xylem sap from the plants was collected by large scale collection carried out by cutting a large number of plants above the hypocotyl and collect the xylem sap which gushed out as a result of capillary pressure by using a pipette. Samples from the collected xylem sap were tested for the targeted metabolites by analytical HPLC-MS system described later in this chapter. The xylem sap was stored at $-20{ }^{\circ} \mathrm{C}$.

\section{High performance liquid chromatography and mass spectrometry}

The collected xylem sap was tested for the targeted secondary metabolites after each collection by injecting samples of the collected xylem in HPLC-MS system. The samples were injected automatically into the system using an auto-sampler (ProStar 410, from Varian, Darmstadt, Germany), the required pressure for the HPLC system was provided by a binary pump system (ProStar 210, Varian), gases were removed from the system using a degassing machine (Degassit, MetaChem Technologies) and the column was kept under the desired temperature by an oven (Jetstream 2 plus, Techlab, Germany). 
The HPLC system was combined with an ion trap mass spectrometer (500- MS, Varian) using an electrospray ionization (ESI) source.10 $\mu \mathrm{L}$ of the sample was injected onto a reverse-phase column Polaris C18-Ether (100 $\times 2 \mathrm{~mm}$ i.d., $3 \mu \mathrm{m}$ particle size, Varian) with a compatible guard column (MetaGuard Polaris C18-Ether, Varian) kept at $40^{\circ} \mathrm{C}$. A gradient of $7 \mathrm{mM}$ acetic acid in $95 \%$ water : $5 \%$ acetonitrile $(A)$ and $7 \mathrm{mM}$ acetic acid in methanol (B) $(0-2.20 \min 90 \%$ A: $10 \%$ B, $2.20-25.0 \mathrm{~min}$ from $90 \% \mathrm{~A}: 10 \%$ B to $2 \% \mathrm{~A}$ : $98 \%$ B, 25-30 min 2\% A: 98\% B was applied, followed by a washing phase and then by reequilibration steps using a flow rate of $0.2 \mathrm{ml} / \mathrm{min}$ ). ESI source was switched either with negative or positive spray polarity, the parameters were set as follows (negative/ positive): needle voltage $-3500 \mathrm{~V} /+5000 \mathrm{~V}$, shield voltage $-600 \mathrm{~V} /+600 \mathrm{~V}$, capillary voltage $-/+50 \mathrm{~V}$ drying gas (nitrogen) $20 \mathrm{psi}\left(138 \mathrm{kPa}\right.$ ) at $250^{\circ} \mathrm{C}$ and nebulizing gas (air) $50 \mathrm{psi}(345 \mathrm{kPa})$. The MS analyzer was operated in full scan mode, mass range $\mathrm{m} / \mathrm{z} 100-800$, scan speed $5000 \mathrm{Da} / \mathrm{sec}, 3$ scans averaged, multiplier voltage set to $1385 \mathrm{~V}$. This method was used in the differential metabolic profiling to identify the $V$. Iongisporum infection specific compounds (Ratzinger, 2008).

\section{Preparation of xylem sap for purification}

A total of $265 \mathrm{ml}$ of xylem sap were collected 3000 plants. The xylem sap centrifuged for 10 minutes to remove all plant residues or any other solid material. The xylem sap was filtered using filter membrane (Viva spin 20, Membrane:10000 MWCO from Sartorius stedim biotech $\mathrm{GmbH}$, Goettingen, Germany). After that, the xylem was defatted with equal amount of cyclo-hexane, the hexane phase was discarded. The xylem sap was frozen at $-80^{\circ} \mathrm{C}$ for three hours. The frozen xylem sap was dried through lyophilization for 80 hours until complete dryness. The dried xylem sap was fluffy and had a powdery texture as shown in Fig 2.

\section{Dry weight of xylem sap}

As a result of collecting xylem from 3000 plants of Brassica napus, $265 \mathrm{ml}$ of xylem sap were collected, each plant resulted in an average of 80-150 $\mu$, the best time of xylem sap collection was in the morning after thorough plant irrigation. 
The dry weight of xylem sap was $1.0280 \mathrm{~g}$, therefore, It is estimated that each plant produced an average of $0.34 \mathrm{mg}$ of dried, filtered, defatted xylem sap.

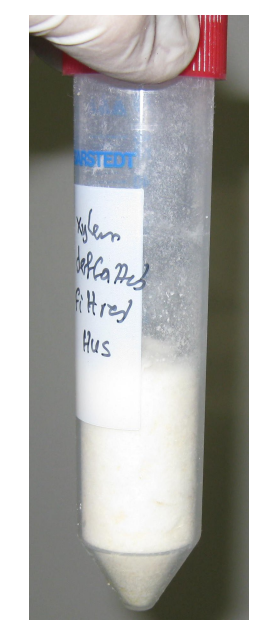

Figure 2. The dried xylem sap.

The fluffy texture of the dried xylem after defatting and the removal of large molecules.

\section{Purification}

The purification was conducted in two steps, in the first step we used. Preparative HPLC system upgraded from the analytical HPLC-MS system, which was used previously to detect these infection specific compounds. In the second step of purification we used semi- preparative system based on using the same column that separated these compounds in HPLC-MS analytical system.

\section{First step of purification with preparative HPLC}

The dried xylem was dissolved in $2.0 \mathrm{ml}$ of methanol/water (50:50, v/v), centrifuged and the supernatant $(1.0 \mathrm{ml})$ was injected (first injection on the Perp-HPLC), the pellet $(\sim 1.0$ $\mathrm{ml}$ ) was then dissolved in an additional $2.0 \mathrm{ml}$ ( $\vee$ final $3.0 \mathrm{ml}$ ), centrifuged and injected in 2 aliquots of $1.5 \mathrm{ml}$ each(second run, conducted with two injections). Purification was conducted using preparative HPLC system, the system pressure was provided by the binary pump system (PU-2086 Semi-Prep JASCO inc, USA), connected to Preparative HPLC column reverse phase Polaris C18-Ether $250 \times 21.2 \mathrm{~mm}$ i.d., particle size of $5 \mu \mathrm{m}$ ( Varian, Darmstadt, Germany) with a compatible guard column Polaris C18-Ether (50 x 
$21.2 \mathrm{~mm}$, Varian). The mobile phase consisted of a binary gradient of $7 \mathrm{mM}$ acetic acid in 95\% water: $5 \%$ acetonitrile $(A)$ and $7 \mathrm{mM}$ acetic acid in methanol (B) $(0-2.20 \mathrm{~min} 90 \% \mathrm{~A}$ : $10 \%$ B, $2.20-25.0$ min from $90 \%$ A: $10 \%$ B to $2 \%$ A: $98 \%$ B, $25-30$ min $2 \%$ A: $98 \%$ B. The eluted fractions were detected by UV/VIS detector (Jasco UV-970, JASCO inc, USA), the wave length was set for detection of the compounds at $A_{280 \mathrm{~nm}}$. The eluted fractions were collected every 30 seconds using fraction collector ( ADVANTEC CHF122SC), The traces of acetic acid were removed from the fractions by adding an adjusted concentration of ammonia solution (Carl Roth $\mathrm{GmbH}$, Germany) the system was run by the software JASCO ChromPass/Galaxie Chromatography Data System. Furthermore the fractions were subjected to HPLC-MS analysis to examine them for the targeted compounds using the analytical system described above.

\section{UPLC combined with PDA-TOF MS Detection}

This detection system was used to examine the pooled sample $(P)$ for its contents of the targeted compound and impurities, and also to examine the fractions eluted from the second step of purification.

Pooled fractions with dry weight of $(0.6 \mathrm{mg})$ labeled as sample $(P)$ in which the metabolite of $\mathrm{m} / \mathrm{z} 613[\mathrm{M}+\mathrm{H}]^{+}$was enriched, were dissolved in $100 \mu \mathrm{l}$ methanol/water $(50: 50, \mathrm{v} / \mathrm{v})$. From this extract $0.2 \mu \mathrm{l}$ were diluted again 1:100 in methanol/water $(50: 50, \mathrm{v} / \mathrm{v}) .4 \mu \mathrm{l}$ of the diluted sample were used for analysis by Ultra Performance Liquid Chromatography (UPLC, ACQUITY UPLC System, Waters Corporation, Milford, USA) coupled with a photo diode array detector (PDA) and an orthogonal time-of-flight mass spectrometer (TOF-MS, LCT Premier, Waters Corporation, Milford, USA). For LC an ACQUITY UPLC HSS T3 column was used at a temperature of $40{ }^{\circ} \mathrm{C}$ and a flow rate of $0.2 \mathrm{ml} / \mathrm{min}$. The following gradient was applied with solvent A (water/formic acid (100:0.1 (v/v)) and solvent B (acetonitrile/formic acid (100:0.1 (v/v)): 0-0.5 min $1 \%$ solvent B, $0.53 \mathrm{~min}$ from $1 \%$ to 20 $\%$ solvent B, 3-8 min from $20 \%$ up to $95 \%$ solvent B, 8-10 min $95 \%$ solvent $B$ and $10-14$ $\min 1 \%$ solvent $B$. The TOF-MS was operated in $W$ optics in positive electrospray ionization (ESI) mode with a mass resolution larger than 10,000. Data was acquired by MassLynx 4.1 software in centroided format over a mass range of $m / z \quad 85-1200$. The capillary and the cone voltage were maintained at $2,700 \mathrm{~V}$ and $30 \mathrm{~V}$ and the solute and the source temperature were $350^{\circ} \mathrm{C}$ and $80^{\circ} \mathrm{C}$, respectively. 
Nitrogen was used as cone $\left(30 / \mathrm{h}^{-1}\right)$ and solvent gas $(800 \mathrm{I} \mathrm{h}$ ). The Dynamic Range Enhancement (DRE) mode was used for data recording. All analysis was monitored by using Leucine-enkephaline $\left(\mathrm{m} / \mathrm{z} 554.2615[\mathrm{M}-\mathrm{H}]^{-}\right.$or $\mathrm{m} / \mathrm{z} 556.2771[\mathrm{M}+\mathrm{H}]^{+}$as well as its ${ }^{13} \mathrm{C}$ isotopomer $\mathrm{m} / \mathrm{z} 555.2615[\mathrm{M}-\mathrm{H}]^{-}$or its double ${ }^{13} \mathrm{C}$ isotopomer $\mathrm{m} / \mathrm{z} 558.2836[\mathrm{M}+\mathrm{H}]^{+}$) as lock spray reference compounds.

\section{Second step of purification (Semi-preparative purification)}

An HPLC purification method has been developed to separate the compound $m / z[M+H]^{+}$ 613 from the impurities. The purification was carried out on an Agilent 1100 HPLC system (Agilent, Waldbronn, Germany) coupled to a diode array detector. For one HPLC run $2 \mu l$ from the $100 \mu$ l sample $(P)$ was injected, which results in 50 runs. A reversed phase (RP)HPLC with the Polaris C18-Ether column $(100 \times 2 \mathrm{~mm}$ i.d., $3 \mu \mathrm{m}$ particle size; Varian, Germany) and the following binary gradient system was used: solvent $A$, water/formic acid $(100: 0.1, v / v)$; solvent $B$, acetonitrile/formic acid $(100: 0.1, v / v)$ with the following gradient program: $10 \%$ solvent $B$ at the starting point, followed by a linear increase of solvent $B$ up to $73 \%$ within $7 \mathrm{~min}$ and by an isocratic run at $73 \%$ solvent $B$ for $5 \mathrm{~min}$. The flow rate was $0.4 \mathrm{ml} / \mathrm{min}$ and for detection of the compounds, $A_{280} \mathrm{~nm}$ was recorded. Fractions were collection in the following time ranges: $2.3-2.8 \mathrm{~min}$ (fraction 1), $2.8-3.7 \mathrm{~min}$ (fraction 2), $3.7-3.9 \mathrm{~min}$ (fraction 3 ) and $3.9-4.3 \mathrm{~min}$ (fraction 4). Fraction 3 contains the compound $m / z 613[\mathrm{M}+\mathrm{H}]^{+}$, which was proved by UPLC TOF MS analysis as shown in Fig 10. 


\section{Results}

\section{Solubility of xylem sap}

The solubility of dried xylem sap was very good. It was possible to dissolve the whole amount of the dried filtered xylem sap in $3 \mathrm{ml}$ of methanol/water $(50: 50, \mathrm{v} / \mathrm{v})$ The dry weight $1.0280 \mathrm{~g}$, was dissolved in $2 \mathrm{ml}$ methanol/water $(50: 50, \mathrm{v} / \mathrm{v})$, centrifuged and the supernatant $(1.0 \mathrm{ml})$ was injected (first injection on the preparative HPLC), the pellet $(\sim 1.0$ $\mathrm{ml}$ ) was then dissolved in $2.0 \mathrm{ml}$ extra (the final volume used to dissolve the whole xylem sap was $3.0 \mathrm{ml}$ ). The purification was conducted by injecting preparative HPLC upgraded from the analytical system as described in the material and methods section of this chapter. The eluted fractions were collected by time every 30 seconds. UV detection of $A_{280}$ ${ }_{n m}$ was recorded for the eluted fractions, furthermore the fractions were subjected to HPLCMS analysis (described in the first step in the purification) to examine the eluted compounds in each fraction. 


\section{First step of purification}

The compound with mass to charge value of $m / z 613[\mathrm{M}+\mathrm{H}]^{+}$was found in the fractions number 38 and 39 in the first injection. The compound was obtained at a retention time of 12.2 minutes as shown in total ion chromatogram in Fig. 2 with the corresponding spectra fraction 39 obtained at a retention time 12.2, containing impurities along with the major compound.

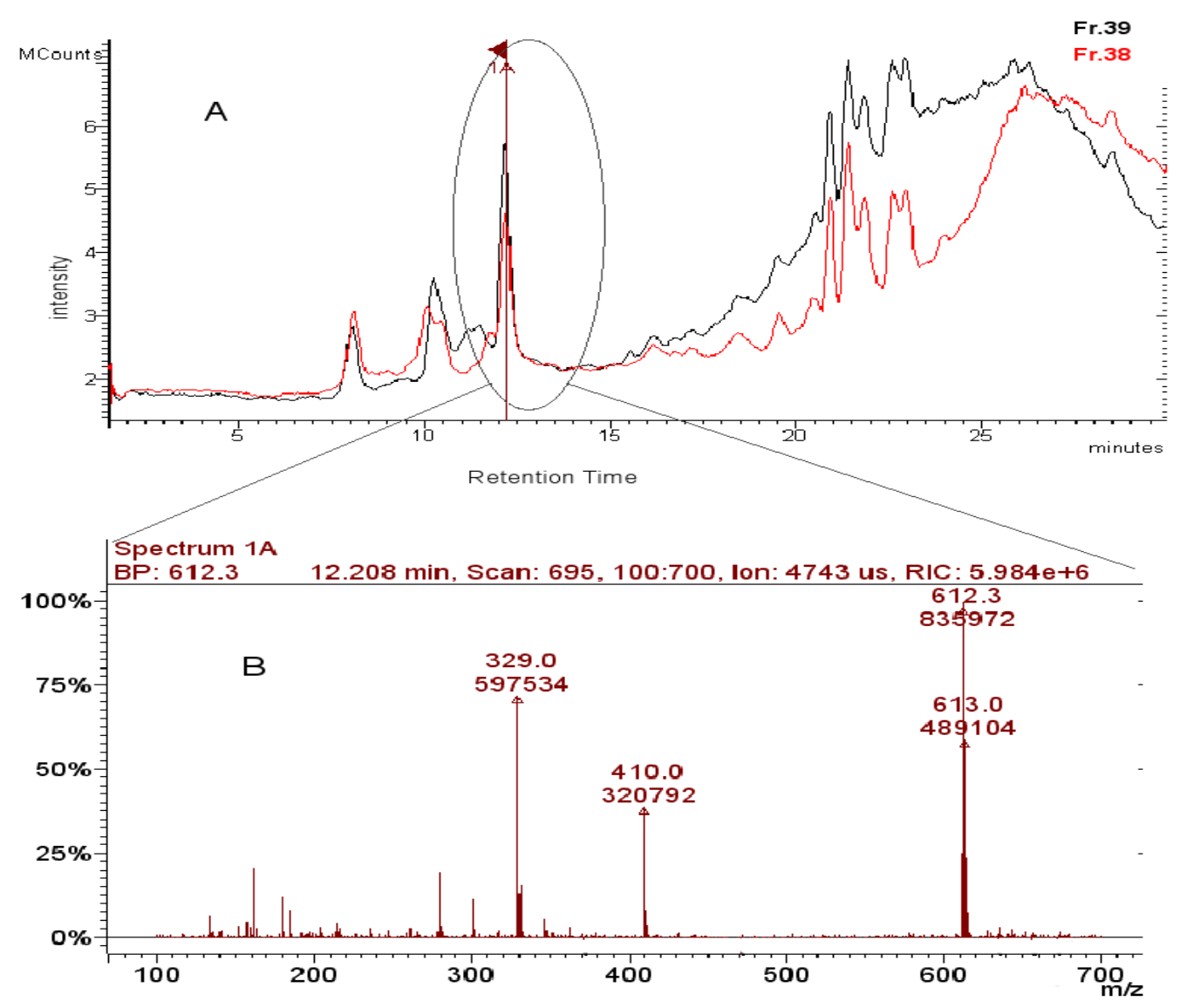

Figure 3. Fraction number 38 and fraction number 39.

A. Total ion chromatogram in positive mode of ESI of fraction number 38 (Fr.38)(red) and fraction number 39 (Fr.39)(black) which were eluted in the first injection and found to be containing the compound of mass to charge $\mathrm{m} / \mathrm{z} 613[\mathrm{M}+\mathrm{H}]^{+}$in positive mode of ESI. B. Corresponding spectra of fraction number 39 at retention time 12.2 minutes in positive mode of ESI. 
The compound of $\mathrm{m} / \mathrm{z} 613[\mathrm{M}+\mathrm{H}]^{+}$was found in fraction 41 in the second injection which was conducted in two runs, and in fraction 2.41 in second run of this injection. The compound was obtained at retention time of 12.2 minute as shown in total ion chromatogram in Fig 2 with the corresponding spectra obtained at at retention time 12.2 in fraction 41 in positive mode of ESI, both TICs from the two runs seemed to be identical and contained impurities along with the major compound $m / z 613[\mathrm{M}+\mathrm{H}]^{+}$of the peak.

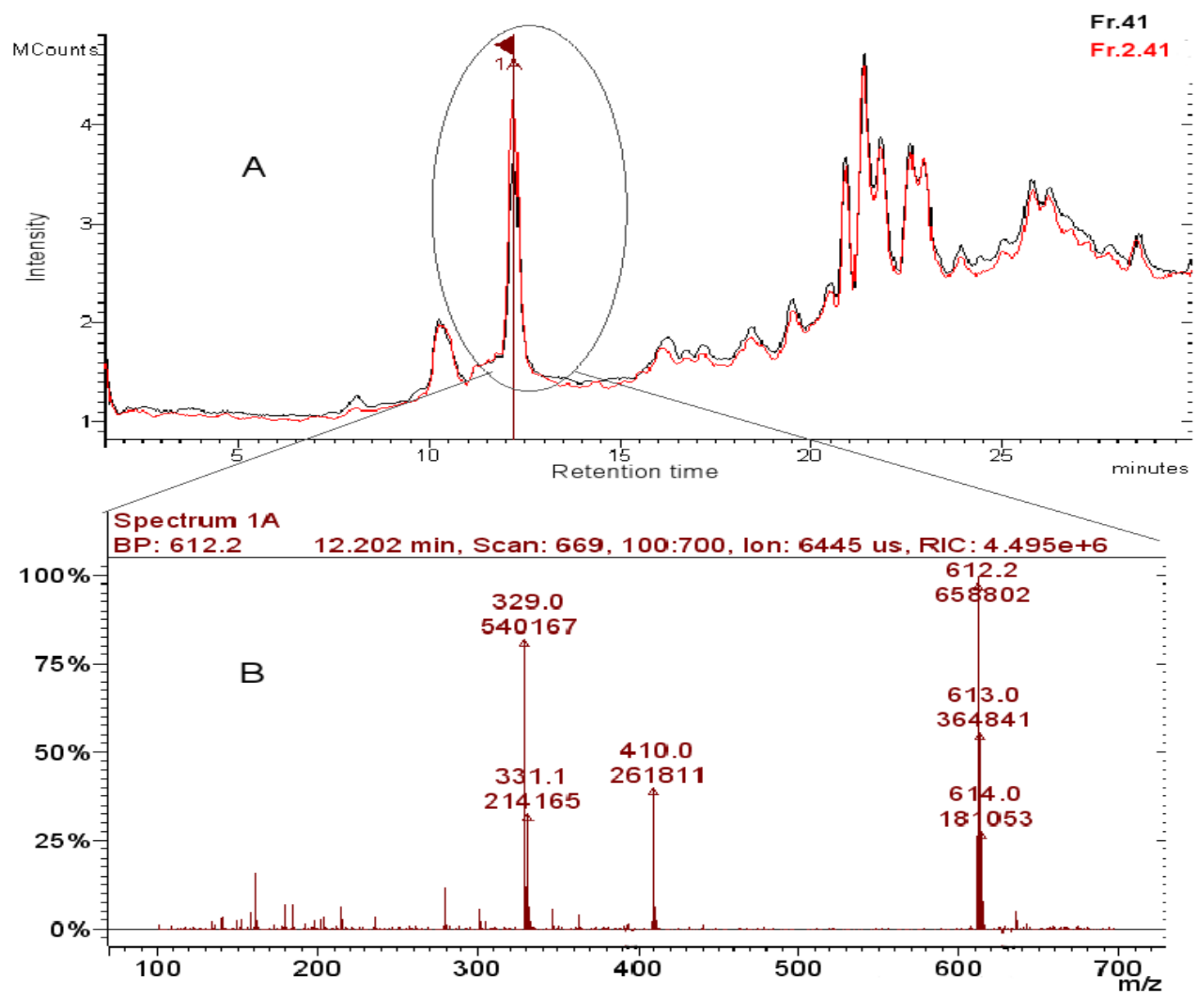

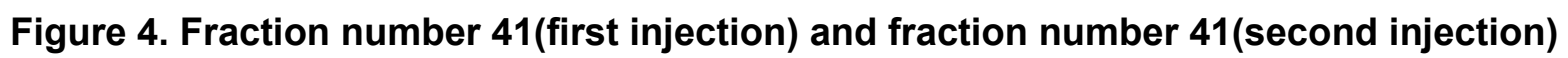

A. Total ion chromatogram in positive mode of ESI of fraction number 41(Fr.41)(black) eluted in first injection, and fraction number 41 labeled as 2.41 (Fr.2.41)(red) which was eluted in the second injection and found to be containing the compound of mass to charge $\mathrm{m} / \mathrm{z} 613[\mathrm{M}+\mathrm{H}]^{+}$in positive mode B. Corresponding spectra of fraction number 41 at retention time 12.2 minutes in positive mode of ESI. 
When the fractions were overlaid with fraction 4, which was eluted at the beginning of gradient and used as a reference to compare the eluted fractions, we observed that the peaks that were eluted after 15 minutes came from methanol. The major peak that came at the retention time 12 minutes of the four fractions, fraction 38, fraction 39 from first injection and fraction 41, fraction 2.41 from second injection contained the compound $\mathrm{m} / \mathrm{z}$ $613[\mathrm{M}+\mathrm{H}]^{+}$. There were three peaks which came at retention times 8,10.3, 11.4 minutes respectively, but they are not present in fraction 4. as shown in Fig 5. The total ion chromatogram of the first injection (fraction 39), and the second injection (41), looked identical apart of the first peak at the retention time of 8 minutes. 


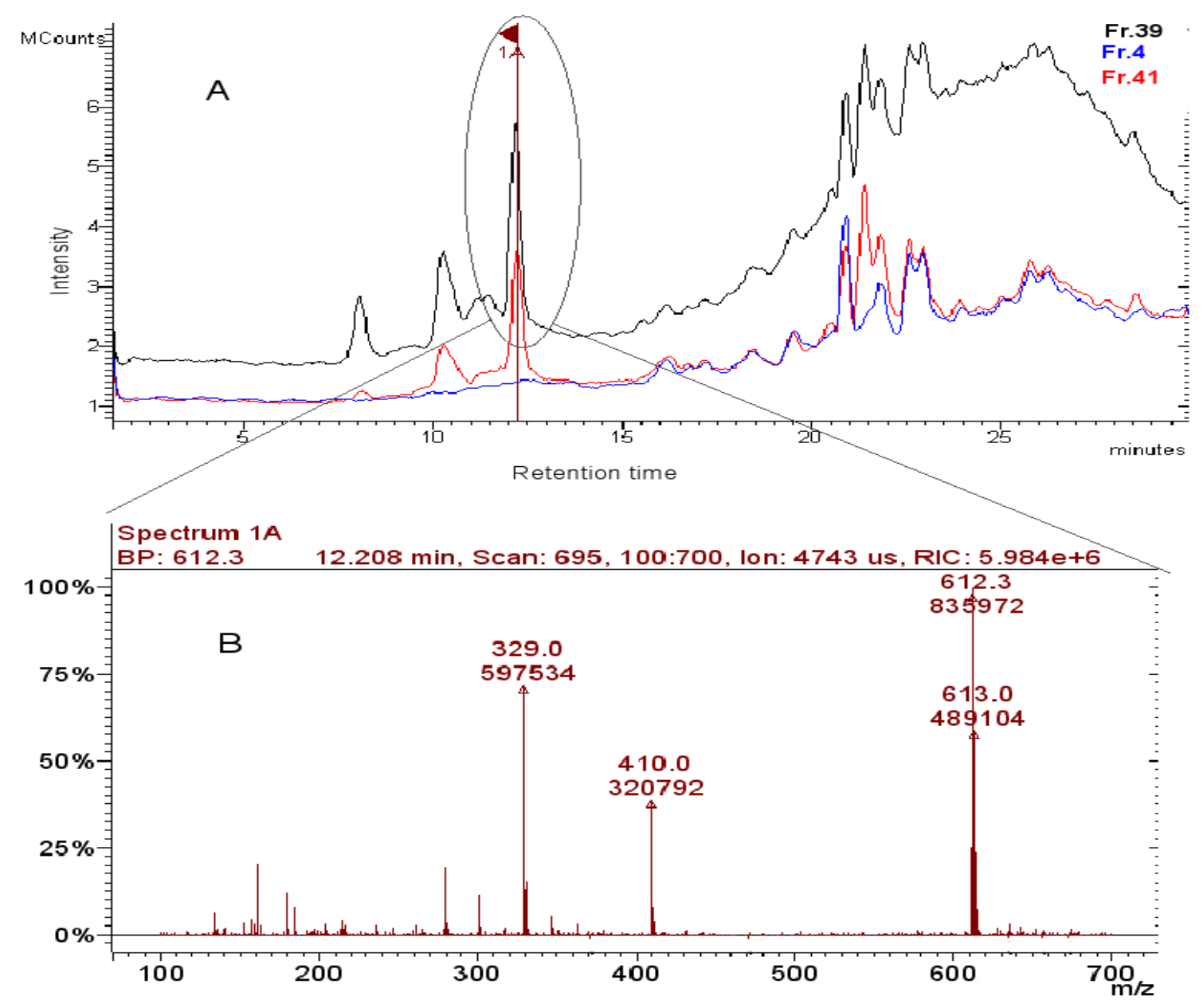

Figure 5: Fraction number 39, fraction number 41, and fraction number 4

A. Total ion chromatogram in positive mode of ESI of fraction number 39 (Fr.39) (black), fraction number 41(Fr.41)(red), overlaid with the fraction number 4 (Fr.4) (blue), from the first injection, which used as reference in positive mode of ESI. B. Corresponding spectra of fraction number 39 at retention time 12.2 minutes in positive mode of ESI. 
The extracted spectrum of the compound $m / z 613[\mathrm{M}+\mathrm{H}]^{+}$showed that all four fractions have similar intensities of $m / z 613[\mathrm{M}+\mathrm{H}]^{+}$. Fig 6 shows the extracted spectra of $613[\mathrm{M}+\mathrm{H}]$ ${ }^{+}$in positive mode of ionization in fraction 38 as an example.

The four fractions: 38 and 39 from the first injection and fractions 41 and 2.41 from the second injection were pooled The dry weight of the four pooled fractions was $(0.6 \mathrm{mg})$ and the sample was labeled as sample $(P)$.

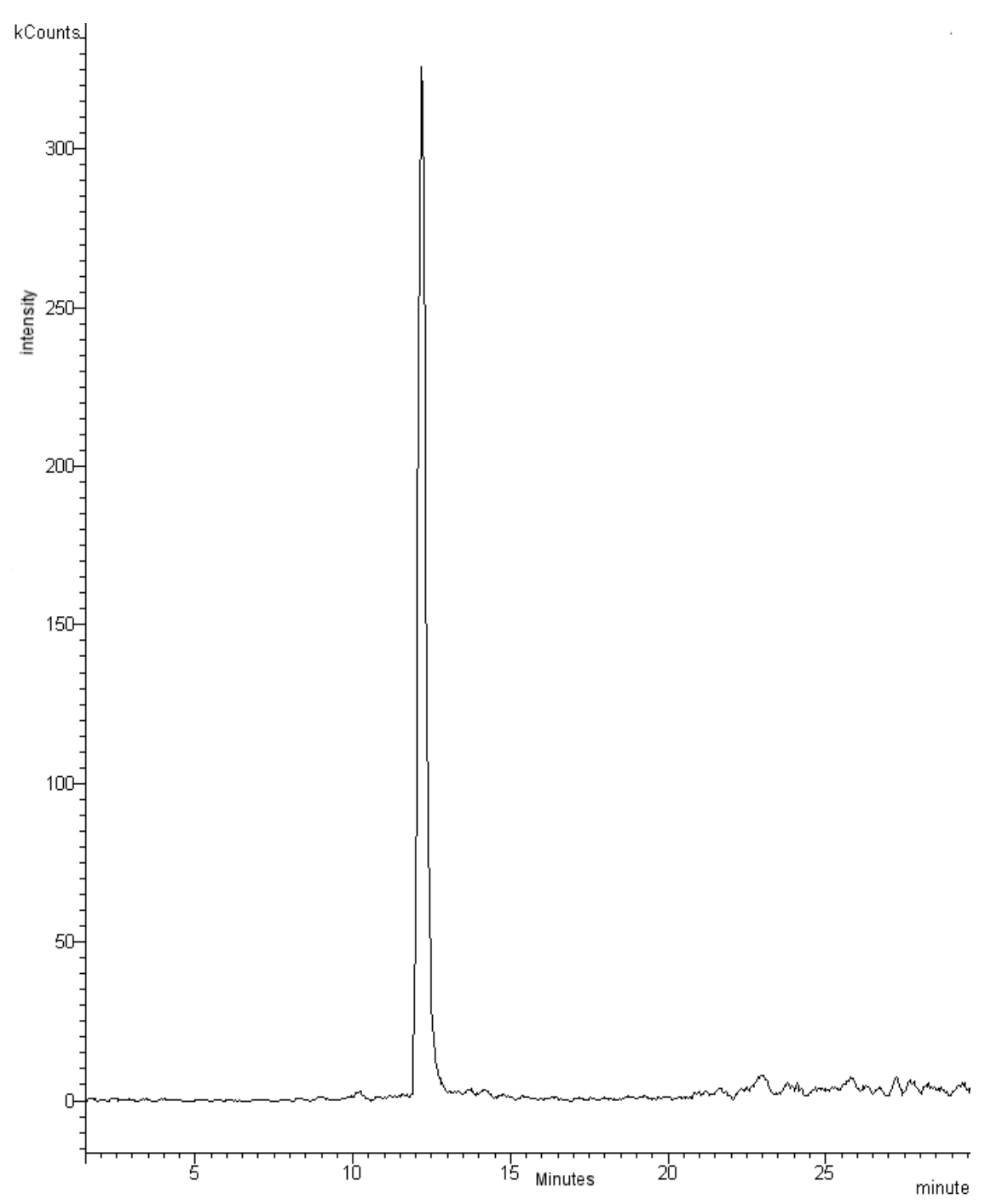

Figure 6. Extracted spectra of the compound of mass to charge $\mathrm{m} / \mathrm{z} 613[\mathrm{M}+\mathrm{H}]^{+}$in fraction number 38. Extracted spectra of the compound of mass to charge $m / z 613[\mathrm{M}+\mathrm{H}]^{+}$in the fraction 38 in positive mode of ESI as an example of the four fractions which contained similar intensity. 


\section{UPLC combined with PDA-TOF- MS Detection of pooled fractions (Sample P)}

The UPLC PDA TOF MS analysis revealed that the sample (P) contained the compound $\mathrm{m} / \mathrm{z} 613[\mathrm{M}+\mathrm{H}]^{+}$in detectable amounts (Fig. 7, extracted ion chromatogram (red line)). The sample contained at least $6-7$ additional compounds enriched during purification, as shown by the results of the UV/VIS analysis (Fig. 7, UV/VIS chromatogram (green line)).

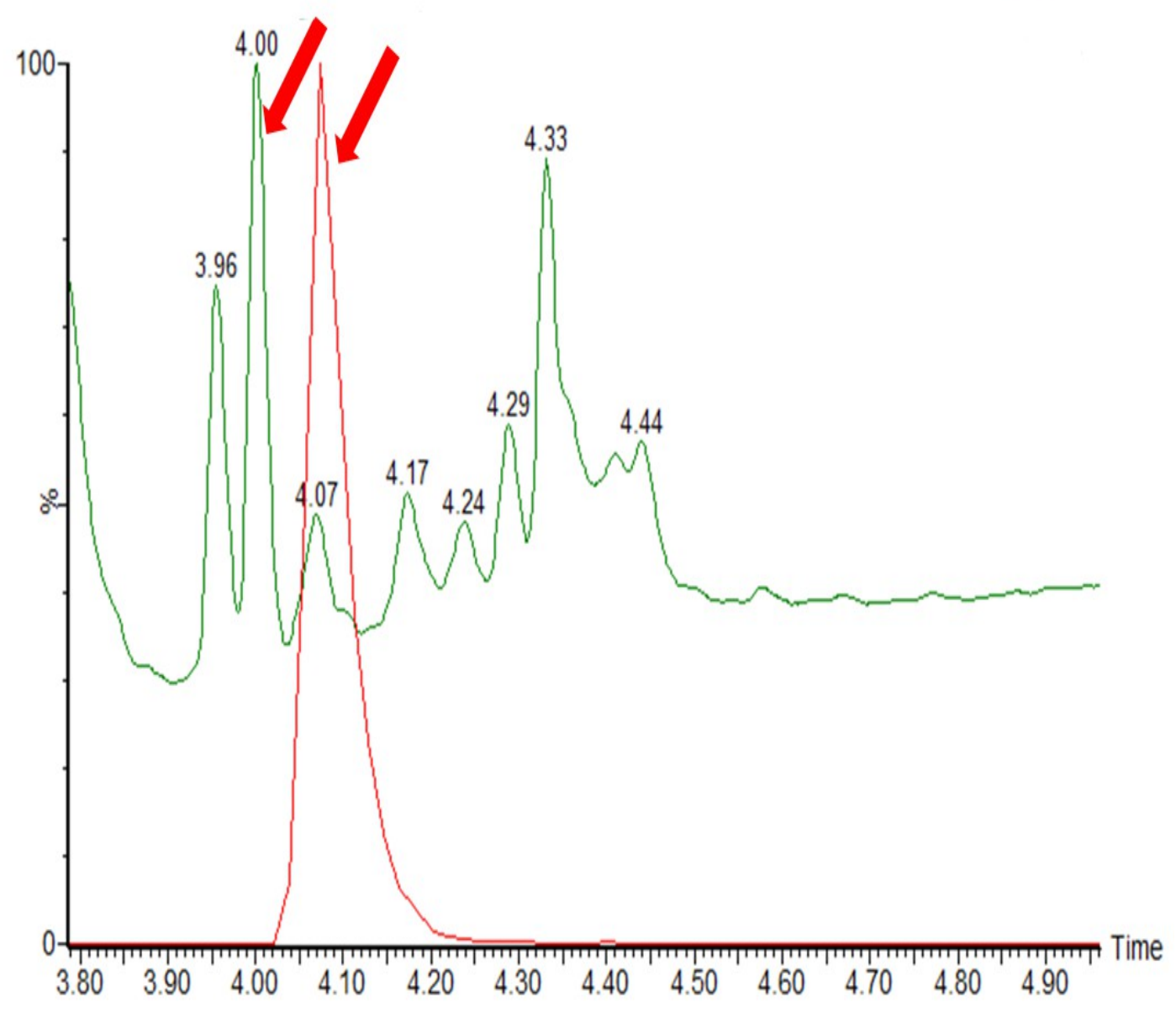

Figure 7: Analysis of sample $P$ (pooled fractions) by UPLC PDA TOF MS measurement.

The UV/VIS chromatogram is shown in (green), while the extracted ion chromatogram of mass to charge $m / z 613.1200$ is shown in (red). 
The exact mass measurement by TOF MS allows to calculate the exact mass of 612.1197 Da for compound $\mathrm{m} / \mathrm{z} 613[\mathrm{M}+\mathrm{H}]^{+}$derived from the molecular ion $\mathrm{m} / \mathrm{z} 613.1281[\mathrm{M}+\mathrm{H}]^{+}$ (Fig. 8). Compound $\mathrm{m} / \mathrm{z} 613[\mathrm{M}+\mathrm{H}]^{+}$seems to undergo "in source" fragmentation, which leads to the fragment of $\mathrm{m} / \mathrm{z} 527.1291[\mathrm{M}+\mathrm{H}]^{+}$(Fig. 8). Additionally a retention time of 4.0 min and two absorption maxima (216 and $287 \mathrm{~nm}$, Fig. 9) were determined for compound $m / z 613[\mathrm{M}+\mathrm{H}]^{+}$.

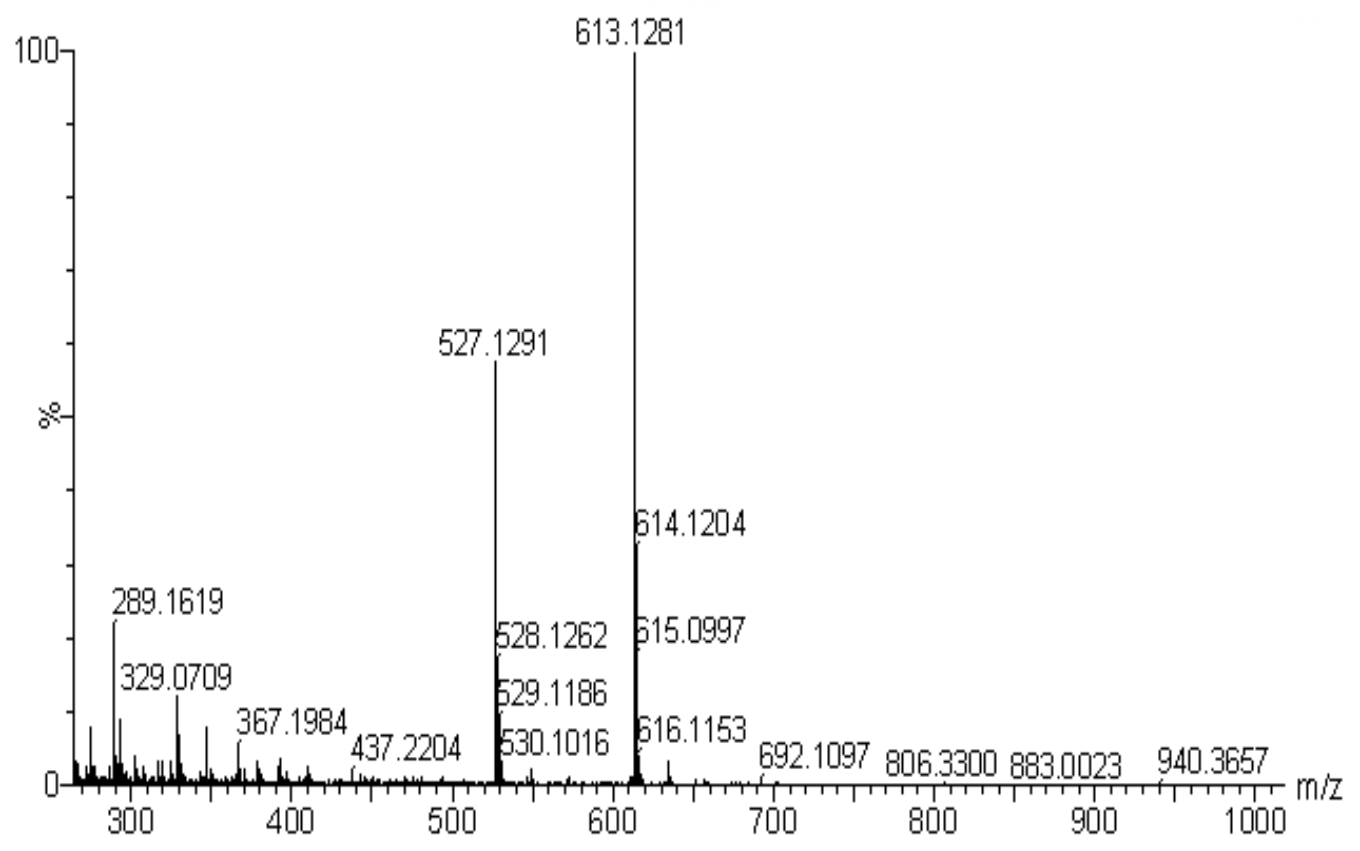

Figure 8: Combined mass spectra of the compound $m / z 613[\mathrm{M}+\mathrm{H}]^{+}$generated by UPLC PDA TOF MS analysis. 


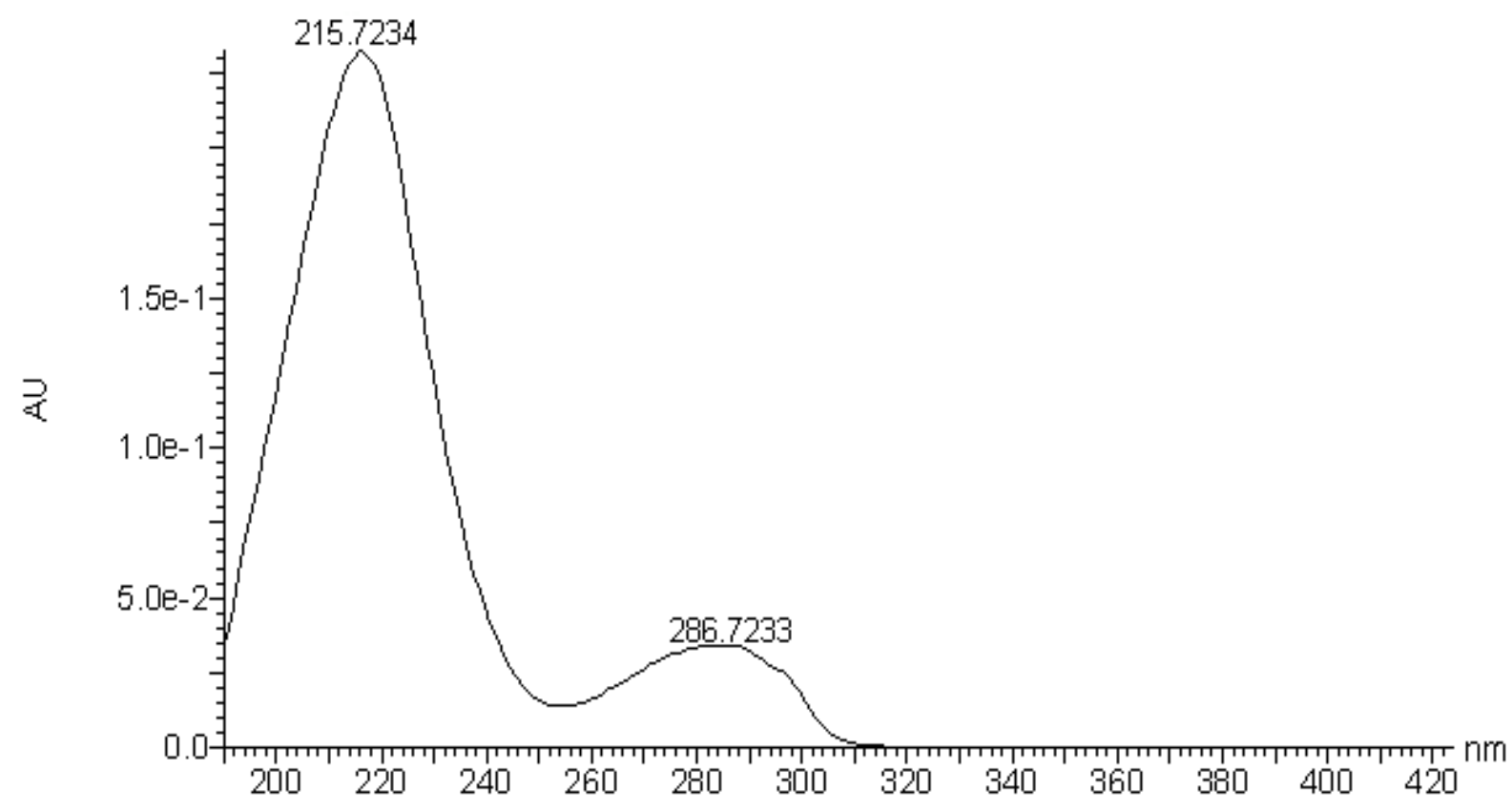

Figure 9: UV/VIS spectra of the compound $m / z 613[\mathrm{M}+\mathrm{H}]^{+}$generated by UPLC PDA TOF MS analysis.

\section{Second step of purification (Semi-preparative purification)}

A semi-preparative HPLC purification method was developed to separate compound $\mathrm{m} / \mathrm{z}$ $613[\mathrm{M}+\mathrm{H}]^{+}$from the impurities (described in the material and methods section). We used the analytical column and collected the fraction as they eluted from column. Eluted compounds, were detected at UV detection of $A_{286} \mathrm{~nm}$.

Fractions were collected in the following time ranges: $2.3-2.8$ min (fraction 1), $2.8-3.7$ $\min$ (fraction 2), $3.7-3.9 \min$ (fraction 3) and $3.9-4.3 \mathrm{~min}$ (fraction 4). Fraction 3 contained the compound $\mathrm{m} / \mathrm{z}[\mathrm{M}+\mathrm{H}]^{+} 613$, which was proved by UPLC TOF MS analysis (Fig.10). By conducting 50 runs and collecting fraction 3 from each run, we could purify the compound $m / z[M+H]^{+} 613$ in one separated fraction which was fraction number 3 as shown in Fig 10 of the UPLC TOF MS analysis. 


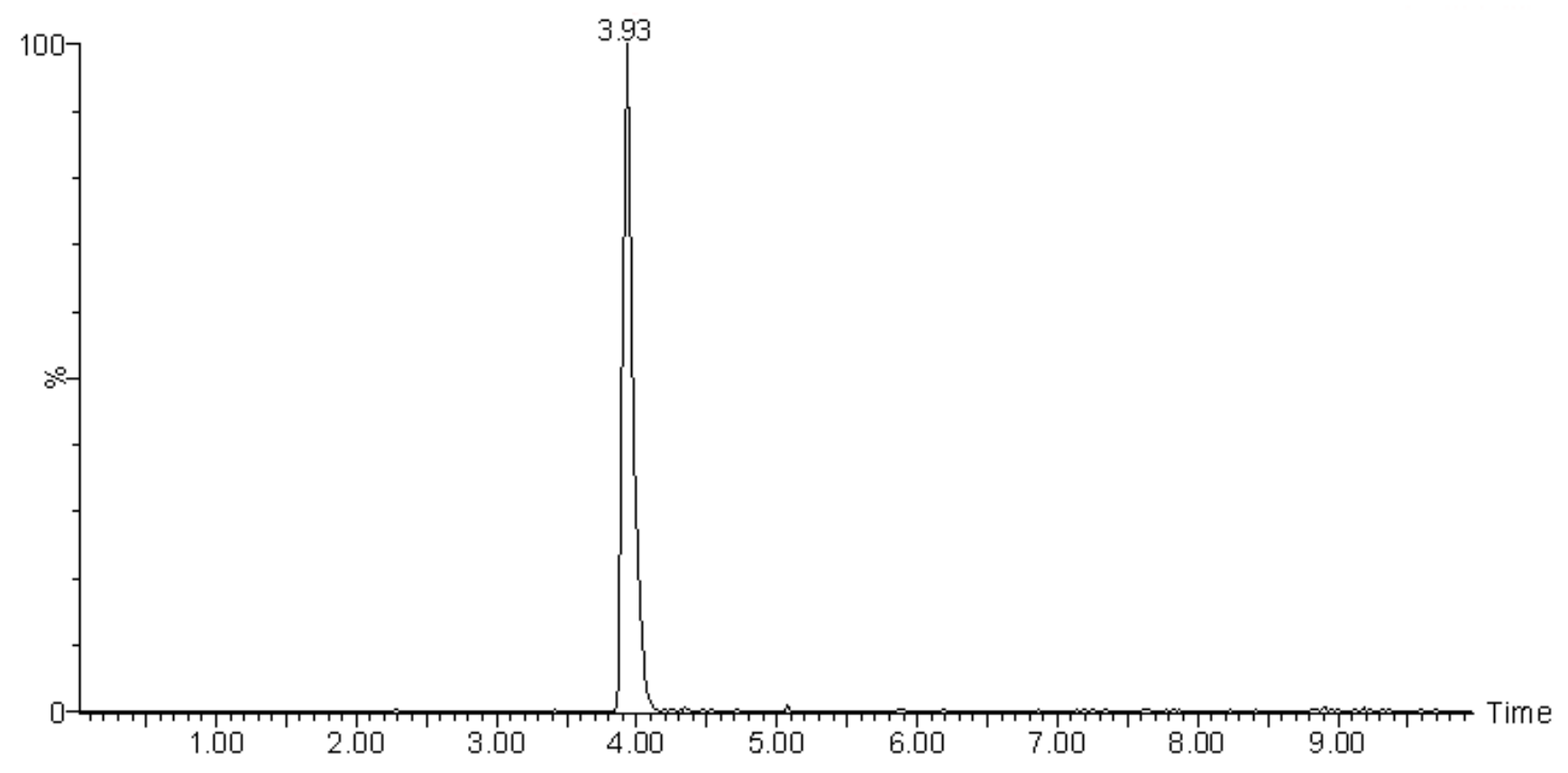

Figure 10:UPLC TOF MS analysis of fraction 3 eluted from the semi preparative HPLC (extracted ion chromatogram of $\mathrm{m} / \mathrm{z}$ 613.1200)

The HPLC fractions number 3 were combined, dried under a stream of nitrogen and used for NMR analysis. The estimation of the dry weight was not possible due to the small amount of the compound obtained.

Due to the small amount of the compound obtained from the semi preparative purification it was not possible to elucidate the structure of the compound of $m / z 613[\mathrm{M}+\mathrm{H}]^{+}$, by using standard NMR spectra. Higher resolution NMR is required for structure elucidation of the purified compound. 


\section{Discussion}

Secondary metabolites that are found specifically in the xylem sap of $V$. longisporum infected plants from Brassica napus were chosen for purification because we presume that they are novel compounds. Their mass to charge ratios did not correspond to the known phytoalexins from Brassica napus. (Pedras, 2011; Ratzinger, 2008) These compounds were not found in supernatant of $V$. longisporum liquid culture when the fungus was grown on medium with a similar composition to xylem sap. So they are believed to be plant originated (Ratzinger, 2008). The purification process could not be facilitated by extracting the compound $\mathrm{m} / \mathrm{z} 613[\mathrm{M}+\mathrm{H}]^{+}$from the xylem sap using an organic solvent, since it was impossible to extract in ethyl acetate and there were large yield losses after extraction in other organic solvents, (Ratzinger, 2008). The isolated compound of $\mathrm{m} / \mathrm{z} 613[\mathrm{M}+\mathrm{H}]^{+}$was the most abundant compound among the compounds which were found to be specific for $V$. longisporum infection in the xylem sap of Brassica napus. The isolated compound $\mathrm{m} / \mathrm{z}$ $613[\mathrm{M}+\mathrm{H}]^{+}$can be detected using the HPLC-MS method in both positive and negative electrospray ionization (ESI).

In another purified fraction of xylem sap, which was obtained by the same method as described above, we noticed that the compound of the $\mathrm{m} / \mathrm{z} 613[\mathrm{M}+\mathrm{H}]^{+}$underwent fragmentation. The intensity of the signal $\mathrm{m} / \mathrm{z} 613[\mathrm{M}+\mathrm{H}]^{+}$was decreased in the sample in favor of an increasing intensity of $m / z 527[\mathrm{M}+\mathrm{H}]^{+}$, which was also one of the infection specific metabolites that Astrid Ratzinger had found in the xylem sap. Both compounds were detected in positive and negative modes of ionization. Furthermore, previous MS $^{2}$ fragmentation on an ion trap revealed that both compounds possessed a common putative block (indole-3-carboxylate) moiety. (Ratzinger, 2008), these fragments were also observed in fragmentation as demonstrated in Fig 8, therefore we believe that the compound of $m / z 527[\mathrm{M}+\mathrm{H}]^{+}$is a fragment of the compound $m / z 613[\mathrm{M}+\mathrm{H}]^{+}$.

Despite of the low complexity of the xylem sap of Brassica napus in comparison to the matrix of the whole plant extraction, the xylem sap contains large molecular compounds like amino acids, proteins and sugars which had to be removed before starting the preparative phase. 
The purification of the compounds from the xylem sap of rapid cycling oilseed rape Brassica napus is not easy due to the very small amount of xylem sap that can be collected from each single plant. The maximum amount of $150 \mu \mathrm{l}$ xylem sap can be extracted from each plant, therefore large scale of xylem sap collection was required. This is very laborious and time consuming process. So new methods for removing the matrix of the whole plant extraction is required. Testing the xylem sap from other plants from Brassicaceae family with larger yields of xylem sap for its contents of these specific $V$. longisporum infection metabolites could be an option, because some phytoalexins in some species can be phytoanticipins in other species, and this depends on the plant and the elicitor (Pedras, 2011, 2010), which is V. longisporum in this case. The isolation and the purification processes were successful in the isolation of the compound with the $\mathrm{m} / \mathrm{z} 613$ $[\mathrm{M}+\mathrm{H}]^{+}$but the quantity of the compound obtained was too low to facilitate standard NMR analysis for structure elucidation, higher resolution NMR analysis is still required to to determine the structure of the compound. Previous chemical characterization of the metabolites $\mathrm{m} / \mathrm{z} 613[\mathrm{M}+\mathrm{H}]^{+}$and the compound of $\mathrm{m} / \mathrm{z} 527[\mathrm{M}+\mathrm{H}]^{+}$based on tandem mass spectrometry showed that the two compounds show neutral loss of of $204 \mathrm{amu}$, which is believed to be tryptophan, and they resulted in same fragments of $\mathrm{m} / \mathrm{z} 162$ which is indole3-carboxylate moiety in $\mathrm{MS}^{3}$, and $\mathrm{m} / \mathrm{z} 118$ in $\mathrm{MS}^{4}$ which can indicated to as indole moiety (Ratzinger, 2008), this was also observed in the purification process, this indicates that the mass of $\mathrm{m} / \mathrm{z} 527.1291[\mathrm{M}+\mathrm{H}]^{+}$is result of a fragmentation of the purified compound of the exact mass $612.1197 \mathrm{Da}$. The phytoalexins cyclobrassinin which previously known as the most abundant phytoalexins in the root of $B$. napus in response to $P$. brassicae (Pedras et al., 2008) posses in its structure, see Fig 4 in Chapter 1; the same moieties of the compound $m / z 613[\mathrm{M}+\mathrm{H}]^{+}$suggested by (Ratzinger, 2008) Therefore we believe that it is a $B$. napus phytoalexin. Fig 11 shows fragmentation of the compounds of $m / z 613[\mathrm{M}+\mathrm{H}]^{+}$ and $m / z 527[\mathrm{M}+\mathrm{H}]^{+}$tandem mass spectrometry conducted by (Ratzinger, 2008). This prediction of the structure of the compound is not unrealistic if we know that most cruciferous phytoalexins have distinctive building blocks from phytoalexins of other plant families including sulfur and nitrogen atoms in addition to the carbon scaffold (Pedras, 2011). 

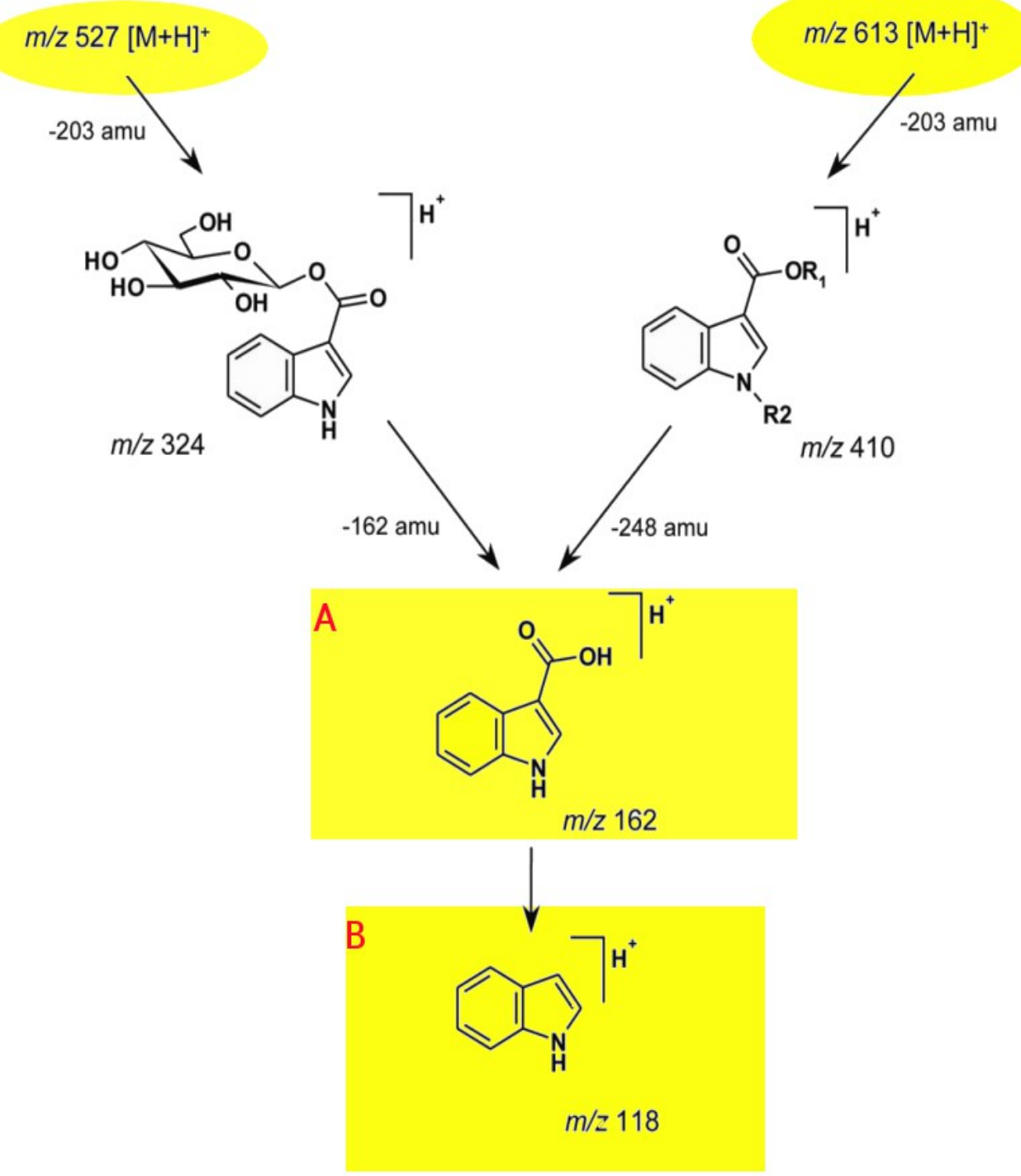

Figure 11: Tandem mass spectrometry of compound $\mathrm{m} / \mathrm{z}[\mathrm{M}+\mathrm{H}]^{+} 613$ and the compound $\mathrm{m} / \mathrm{z}$ $527[\mathrm{M}+\mathrm{H}]^{+}$conducted by (Ratzinger, 2008)(modified after Ratzinger, 2008). Both compounds shared common neutural loss of $204 \mathrm{amu}$, which is believed to be tryptophan, A: Fragments of $\mathrm{m} / \mathrm{z}$ 162 which is indole-3-carboxylate moiety in $\mathrm{MS}^{3}$, B: Fragments of $m / z 118$ in $\mathrm{MS}^{4}$ which indicated as indole moiety.

The purified compound can be a novel phytoalexin of $B$. napus elicited by $V$. longisporum and it is one of cyclobrassinin derivatives. Another suggestion is that the purified compound is a detoxified form of a known cruciferous phytoalexins.

The NMR spectra is still the only analysis which enables us to accurately elucidate the structure of the purified compound. Structure elucidation, using high resolution NMR spectra analysis of this purified compound is being investigated. 


\section{References}

Clay, N.K., 2011. Chemical diversity on display in the plant innate immune systems of closely-related species. New Phytol. 192, 566-569.

Darvill, A.G., Albersheim, P., 1984. Phytoalexins and their elicitors-A defense against microbial Infection in plants. Annu. Rev. Plant Physiol. 35, 243-275.

Dunker, S., Keunecke, H., Steinbach, P., Von Tiedemann, A., 2008. Impact of Verticillium longisporum on Yield and Morphology of Winter Oilseed Rape (Brassica napus) in Relation to Systemic Spread in the Plant. J. Phytopathol. 156, 698-707.

Eynck, C., Koopmann, B., Grunewaldt-Stoecker, G., Karlovsky, P., Tiedemann, A. von, 2007. Differential interactions of Verticillium longisporum and V. dahliae with Brassica napus detected with molecular and histological techniques. Eur. J. Plant Pathol. 118, 259274.

Eynck, C., Koopmann, B., Karlovsky, P., von Tiedemann, A., 2009. Internal Resistance in Winter Oilseed Rape Inhibits Systemic Spread of the Vascular Pathogen Verticillium longisporum. Phytopathology. 99, 802-811.

Floerl, S., Druebert, C., Majcherczyk, A., Karlovsky, P., Kües, U., Polle, A., 2008. Defence reactions in the apoplastic proteome of oilseed rape (Brassica napus var. napus) attenuate Verticillium longisporum growth but not disease symptoms. Bmc Plant Biol. 8, 129.

Hart, S.V., Kogan, M., Paxton, J.D., 1983. Effect of soybean phytoalexins on the herbivorous insects mexican bean beetle and soybean looper. J. Chem. Ecol. 9, 657-672.

Heale, J.B., Karapapa, V.K., 1999. The Verticillium threat to canada's major oilseed crop: canola. Can. J. Plant Pathol. 21, 1-7.

Huisman, O.C., 1982. Interrelations of Root Growth Dynamics to Epidemiology of RootInvading Fungi. Annu. Rev. Phytopathol. 20, 303-327.

Johansson, A., Goud, J.-K.C., Dixelius, C., 2006. Plant Host Range of Verticillium longisporum and Microsclerotia Density in Swedish Soils. Eur. J. Plant Pathol. 114, 139149. 
Kamble, A., Koopmann, B., von Tiedemann, A., 2012. Induced resistance to Verticillium longisporum in Brassica napus by $\beta$-aminobutyric acid. Plant Pathol.62, 552-561.

Karapapa, V.K., Bainbridge, B.W., Heale, J.B., 1997. Morphological and molecular characterization of Verticillium longisporum comb, nov., pathogenic to oilseed rape. Mycol. Res. 101, 1281-1294.

Keen N. T., Bruegger B., 1977. Phytoalexins and Chemicals That Elicit Their Production in Plants, in: Host Plant Resistance to Pests, ACS Symposium Series. American Chemical Society, pp. 1-26.

Mol, L., Riessen, H.W. van, 1995. Effect of plant roots on the germination of microsclerotia of Verticillum dahliae. Eur. J. Plant Pathol. 101, 673-678.

Pedras, M.S.C., Adio, A.M., Suchy, M., Okinyo, D.P.O., Zheng, Q.-A., Jha, M., Sarwar, M.G., 2006. Detection, characterization and identification of crucifer phytoalexins using high-performance liquid chromatography with diode array detection and electrospray ionization mass spectrometry. J. Chromatogr. A 1133, 172-183.

Pedras, M.S.C., Okanga, F.I., Zaharia, I.L., Khan, A.Q., 2000. Phytoalexins from crucifers: synthesis, biosynthesis, and biotransformation. Phytochemistry 53, 161-176.

Pedras, M.S.C., Yaya, E.E., 2010. Phytoalexins from Brassicaceae: news from the front. Phytochemistry $71,1191-7$.

Pedras, M.S.C., Yaya, E.E., Glawischnig, E., 2011. The phytoalexins from cultivated and wild crucifers: chemistry and biology. Nat. Prod. Rep. 28, 1381-405.

Pedras, M.S.C., Zheng, Q.-A., Strelkov, S., 2008. Metabolic Changes in Roots of the Oilseed Canola Infected with the Biotroph Plasmodiophora brassicae: Phytoalexins and Phytoanticipins. J. Agric. Food Chem. 56, 9949-9961.

Pegg, G.F., Brady, B.L., 2002. Verticillium wilts 2002 pp. UK.

Ratzinger, A., 2008. Development and application of LC-MS-based differential metabolic profiling in plant systems. Georg-August-Universität Göttingen, Göttingen, Germany.

Romagnolo, D.F., Davis, C.D., Milner, J.A., 2012. Phytoalexins in cancer prevention. Front. Biosci. J. Virtual Libr. 17, 2035-2058. 
Zeise, K., Tiedemann, A. von, 2001. Morphological and Physiological Differentiation among Vegetative Compatibility Groups of Verticillium dahliae in Relation to $V$. longisporum. J. Phytopathol. 149, 469-475.

Zeise, K., Von Tiedemann, A., 2002. Host Specialization among Vegetative Compatibility Groups of Verticillium dahliae in Relation to Verticillium longisporum. J. Phytopathol. 150, 112-119.

Zhou, L., Hu, Q., Johansson, A., Dixelius, C., 2006. Verticillium longisporum and V. dahliae: infection and disease in Brassica napus. Plant Pathol. 55, 137-144. 


\title{
Chapter 3: The effect of Verticillium longisporum infection on the growth of Agrobacterium tumefaciens and Saccharomyces cerevisiae in xylem sap of Brassica napus
}

\author{
Husam Ibrahem Aroud and Petr Karlovsky \\ Molecular Phytopathology and Mycotoxin Research Unit, Department of Crop Sciences, University of \\ Göttingen, Grisebachstrasse 6, 37077 Göttingen, Germany
}

\begin{abstract}
Verticillium longisporum is a vascular fungus which infects plants from the Brassicaceae family like oilseed rape Brassica napus and Arabidopsis thaliana through the roots. It colonizes the vascular system of the host plants and stays restricted to it during the biotrophic phase of its life cycle. Xylem is the environment where the biochemical interactions between the pathogen and the host plant take place. Xylem sap contains organic acids, amino acids and sugars on which microorganisms can grow. The aim of the study was to understand the impact of the infection of $V$. longisporum on the growth of two microorganisms, the bacterium $A$. tumefaciens and the yeast Saccharomyces cerevisiae when they grow in xylem sap taken from oilseed rape Brassica napus. We developed an assay using xylem sap as medium and a moisture chamber. An inhibition effect on $A$. tumefaciens was significantly observed after 24 hours of incubation; this effect was not observed against $S$. cerevisiae. The inhibition effect was eliminated when proteins of molecular weight larger than $3 \mathrm{kDa}$ were removed from xylem sap using membrane filtration. Small molecules in xylem sap of Brassica napus appear to have a limited effect on the studied microorganisms.
\end{abstract}

Key words: Verticillium longisporum, Brassic napus, xylem sap, Agrobacterium tumefaciens, Saccharomyces cerevisiae. 


\section{Introduction}

Oilseed rape (Brassica napus) is a major crop from the plant family Brassicacae. It is grown in many parts of the world like Canada, China, India and Europe for its nutritious edible oil and for other uses including the production of animal fodders and biofuel. The recent decades have witnessed a noticeable increase in the cultivated area of oilseed rape in Europe because of the increasing demands of biofuel due to the limited natural resources of energy in the world. Oilseed rape is mainly cultivated in Northern Europe. Soil borne diseases can lead to high losses in the yield of oilseed rape (Dunker et al., 2008). $V$. longisporum is the most prevalent soil-borne pathogen of oilseed rape in Sweden. It is one of the prevalent pathogens in Northern Germany (Johansson et al., 2006; Siebold and Tiedemann, 2012). The pathogen is considered as potential threat to oilseed crop production in Canada (Heale and Karapapa, 1999). V. longisporum belongs to genus Verticillium spp., which are soil-borne vascular plant pathogens from the fungal division Ascomycota. Many of them are responsible for vascular diseases on a wide range of important crops, particularly in sub-tropical and moderate regions (Fradin and Thomma, 2006; Pegg and Brady, 2002). Morphological and molecular characterization of $V$. longisporum differentiated it from $V$. dahliae which was previously believed that it is the casual agent of Verticillium disease on oilseed rape (Karapapa et al., 1997).Recent studies confirmed that $V$. longisporum is diploid and hybrids evolved through four different lineages and from three different parental species including V. dahliae (Inderbitzin et al., 2011). The pathogenicity of $V$. longisporum is specific to cruciferous host plants (Zeise and von Tiedemann, 2002). V. longisporum, is the casual agent of Verticillium disease on B. napus and can cause losses up to $80 \%$ of the yield (Dunker et al., 2008; Karapapa et al., 1997), and what makes the infection a serious problem is the fact that there is more no available known chemical control of the disease. In the dormant phase of its life cycle, $V$. longisporum can survive in the soil for many years by forming thick-walled melanised microsclerotia (Heale and Karapapa, 1999), germination is stimulated by plant root exudates followed by fungus penetration of the host roots, the fungus spreads in the xylem vessels systemically colonizing the xylem vessels and stays limited to them during the biotrophic phase of its life cycle (Eynck et al., 2007; Zhou et al., 2006). The fungus colonizes plant xylem which is the channel that enables plant to transport water and soluble minerals from the roots to the shoots. Despite of the fact that xylem sap in plants is 
considered to be poor in terms of nutritious medium (López-Millán et al., 2000). V. longisporum benefits from the amino acids, carbohydrates, and minerals of the xylem sap and uses them as nutrient source. However, $V$. longisporum infection does not limit the nutrients supply of the plant compared to that of healthy plants, therefore symptoms observed on oilseed rape is not due to nutrient deprivation caused by infection (Floerl et al., 2008). B. napus xylem sap composition of amino acids is still unknown, but previous studies showed that glutamate followed by glutamine, in addition to aspartic acid, and serine are among the most abundant amino acids in mesophyll cells of the leaves of $B$. napus (Tilsner et al., 2005). Xylem sap is the environment where the biochemical interactions between the pathogen and the host plants take place (Ratzinger, 2008). Plants may defend themselves against fungal infection by producing phytoalexins, (Darvill and Albersheim, 1984; VanEtten et al., 1994). The cruciferous host plants produce a wide range of structurally related phytyoalexins (Pedras, 2011, 2010). Therefore, it is expected that xylem sap from infected plants contain these antimicrobial compounds. Secondary metabolites specific for $V$. longisporum infection were detected in the xylem sap of infected $B$. napus plants, but none of them has the same mass of any of the known cruciferous phytoalexins (Ratzinger, 2008). Furthermore, changes in phytohormones were observed. Salicylic acid (SA) and salicylic acid glucoside (SAG) concentrations in the xylem sap of $B$. napus were increased after infection with $V$. Iongisporum. Their concentrations were correlated with disease severity, whereas jasmonic acid (JA) and abscisic acid (ABA) levels in xylem sap did not show any changes after infection. (Ratzinger et al., 2009). Deposition of phenolics bind to the cell wall and lignins, lignin-like modification within the vascular system can also be observed after infection with $V$. longisporum, as they play an important role in resistance mechanism against the invading fungus (Eynck et al., 2009; Obermeier et al., 2013). Analyzing xylem sap of $B$. napus for its content of proteins revealed that it contains 69 proteins, the best known are chitinases and the disease resistance response protein (PR-1), in addition to peroxidases which are suggested to play a role in the generation of reactive oxygen species and the regulation of $\mathrm{H}_{2} \mathrm{O}_{2}$ in plants (Kehr et al., 2005). A later study made it clear that infection with $V$. Iongisporum significantly enhances 12 proteins in the xylem sap of $B$. napus, the proteins identified

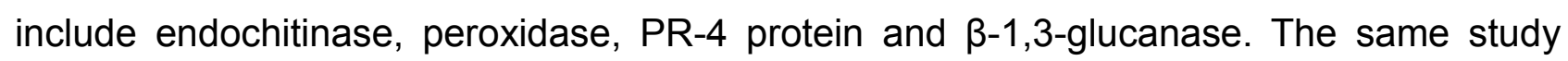
showed that xylem sap taken from infected $B$. napus inhibits the growth of $V$. longisporum 
(Floerl et al., 2008). In a later study, the fungal growth of $V$. longispurm was drastically inhibited during growth in xylem sap from infected or healthy $B$. napus plants, but this inhibition effect was not observed when the large molecules, which have molecular weights larger than $3 \mathrm{kDa}$ were separated from the xylem sap by filter membrane. Furthermore, these macromolecules were able to inhibit the fungi even when they were added externally to standard complete medium, which gives clear evidence that plants secret these proteins in the xylem to inhibit the fungus (Singh et al., 2012). In response, the fungus reacts to the presence of xylem sap of $B$. napus by producing proteins, all of the upregulated ones are involved in the protection against oxidative stress. The most important one of them is catalase peroxidase which plays the key role protecting the fungus from reactive oxygen species (ROS) in this saprophytic phase of its life cycle (Singh et al., 2012).

\section{Aim of the work}

The aim of the study was to investigate the impact of the infection of $V$. longisporum and the small molecules related to infection on the growth of two microorganisms, when they grow in the xylem sap taken from the oilseed rape Brassica napus. The experiment examined whether there is an inhibition effect induced by $V$. longisporum infection, and if this inhibition effect is related to small molecules present in xylem sap.

\section{Authors' contributions}

Husam Ibrahem Aroud carried out all experiments, xylem sap extraction, Petr Karlovsky designed and supervised the study and corrected the manuscript.

Some parts of the assay were conducted using unfiltered and macromolecules filtered xylem sap taken from $V$. longisporum infected and uninfected $B$. napus, plants provided by Christine Druebert, from the research group of Prof. Dr. Andrea Polle (Department of forest botany and tree physiology, Georg-August University, Göttingen, Germany). 


\section{Materials and Methods}

\section{Xylem sap}

The assay was conducted on xylem sap from B. napus as a medium to grow the aforementioned microorganisms. Xylem sap was extracted from healthy and $V$. longisporum infected $B$. napus plants. Xylem sap was either complete, unfiltered or macromolecules filtered by using a $3000 \mathrm{MWCO}$ filter membrane.

\section{Unfiltered xylem sap}

\section{Brassica napus plants}

Oilseed rape plants used in this experiment were rapid-cycling rape, (Brassica napus var. napus, genome ACaacc), the seeds were provided by Paul H. Williams (Department of Plant Biology, University of Wisconsin-Madison, Crucifer Genetics Cooperative, Stock number 5 ). The seeds were subjected to surface sterilization by dipping them in $70 \%$ ethanol for 30 seconds, followed by rinsing them with tab water three times for 30 seconds before sowing. The seeds were sown in sterilized silica sand and left to germinate under semi sterile, controlled condition of $22 / 20^{\circ} \mathrm{C}$ day/ night), $15 \mathrm{~h}$ day long and $60 \%$ humidity, with regular irrigation.

\section{Verticillium longisporum isolate $\mathbf{4 3}$}

Verticillium longisporum VL 43 isolated from Brassica napus plants grown in northern Germany as described in (Zeise and Tiedemann, 2001). Fungal stocks with $1 \times 10^{6}$ spore/ $\mathrm{ml}$ were stored at $\left(-80^{\circ} \mathrm{C}\right)$ in $25 \%$ glycerol. 


\section{Preparation of Verticillium longisporum inoculum}

$500 \mu \mathrm{l}$ of Verticillium longisporum spore suspension $\left(1 \times 10^{6}\right.$ spore/ml ) was added to $300 \mathrm{ml}$ Czapek Dox broth medium. The inoculated broth was incubated at $23{ }^{\circ} \mathrm{C}$ on a rotary shaker at $100 \mathrm{rpm}$ and kept in the dark. A sterile sieve was used after10-14 days to filter the fungal mycelia. Spore concentration was determined by counting spores using a haemocytometer (Thoma) and then diluted to $1 \times 10^{6}$ spore $/ \mathrm{ml}$.

\section{Inoculating and growing the plants}

Rape seedlings were grown for 7-10 days in autoclaved silica sand, then they were infected by root dipping with the $V$. longisporum spore suspension, with concentration of $1 \times 10^{6} \mathrm{spore} / \mathrm{ml}$ for 40 minutes. Control seedlings were dipped in sterile water for the same period of time. After that, each single seedling was transferred to a pot of sterile sand-soil mixture (1:1). Both infected and healthy plants were grown in climatic chamber for 28 (dpi) under constant conditions of $22 / 20{ }^{\circ} \mathrm{C}$ day/ night, $15 \mathrm{~h}$ day long and $60 \%$ humidity with regular irrigation.

\section{Xylem sap extraction}

Plants were grown for $28 \mathrm{dpi}$ and they were thoroughly irrigated before xylem sap extraction. The plants stems were cut above the hypocotyl and the xylem sap which gushed out under capillary pressure was collected using a pipette, the xylem sap was frozen for storage at $-20^{\circ} \mathrm{C}$.

\section{Macromolecules filtered xylem sap}

Some parts of the assay were conducted using complete, unfiltered xylem sap and macromolecules filtered xylem sap; by using a 3000 MWCO filter membrane, taken from $V$. longisporum infected and healthy $B$. napus plants. The xylem sap was provided by Christine Druebert, from research group of Prof. Dr. Andrea Polle (Department of Forest Botany and Tree Physiology, Georg-August University, Göttingen, Germany). 


\section{Microbial isolates and media}

\section{Microbial isolate}

Agrobacterium tumefaciens strain AGL1 was provided by Dr. Susanne Frick, Leibniz Institute of Plant Biochemistry (Halle/Saale, Germany). Saccharomyces cerevisiae strain CEN-PK2-1C was provided by Dr. Wilfried Kramer (Institute for Microbiology and Genetics, Göttingen, Germany); the source of the yeast is described in the paper (Entian et al., 1999).

\section{LB Medium}

LB-medium $(1 \mathrm{~L})$ was prepared by adding:10 g Tryptone (Carl Roth $\mathrm{GmbH}$, Karlsruhe , Germany), $5 \mathrm{~g}$ yeast extract (AppliChem GmbH, Darmstadt, Germany) ,10 g NaCl, dissolved in $950 \mathrm{~mL}$ water, the $\mathrm{pH}$ of the medium was adjusted to 7.0 and the final volume was up to 1 liter.(for petri dishes preparation, $20 \mathrm{~g}$ Agar (Carl Roth $\mathrm{GmbH}$, Karlsruhe, Germany) were added to the liquid medium) The medium was autoclaved with a liquid cycle for $18 \mathrm{~min}$ at $120^{\circ} \mathrm{C}$. No antibiotics were added. Petri dished of LB medium were made by pouring $20 \mathrm{ml}$ of the medium to each petri dish and left for solidification.

\section{YPD Medium}

YPD medium $(1 \mathrm{~L}$ ) was prepared by adding $20 \mathrm{~g}$ of peptone (Carl Roth $\mathrm{GmbH}$, Karlsruhe, Germany) to $10 \mathrm{~g}$ yeast extract (AppliChem $\mathrm{GmbH}$, Darmstadt, Germany) and $20 \mathrm{~g}$ Dextrose (Carl Roth $\mathrm{GmbH}$, Karlsruhe, Germany).to $1000 \mathrm{ml}$ with deionized water. (for petri dishes preparation, $20 \mathrm{~g}$ Agar (Carl Roth $\mathrm{GmbH}$, Karlsruhe, Germany), Autoclaved with a liquid cycle for $15 \mathrm{~min}$ at $120^{\circ} \mathrm{C}$. No antibiotics were added. Petri dished were prepared in similar method to LB medium.

\section{SXM medium}

Simulated xylem fluid medium (SXM) was used for bacteria or yeast, this was diluted and prepared before transfer to the xylem sap. Preparation of the medium is done as described by (Neumann and Dobinson, 2003). 


\section{Moisture chamber}

A moisture chamber was designed to prevent the xylem sap from drying. It was prepared by placing glass petri dish inside a bigger petri dish. Sterilized Eppendorf tubes caps were used as containers of xylem sap in which the microorganism were grown. The moisture chambers were sterilized by autoclaving for $18 \mathrm{~min}$ at $120^{\circ} \mathrm{C}$, the chambers left to be cooled and then sterile water was added under sterile conditions. Fig. 1 shows the moisture chamber which was used in this experiment.

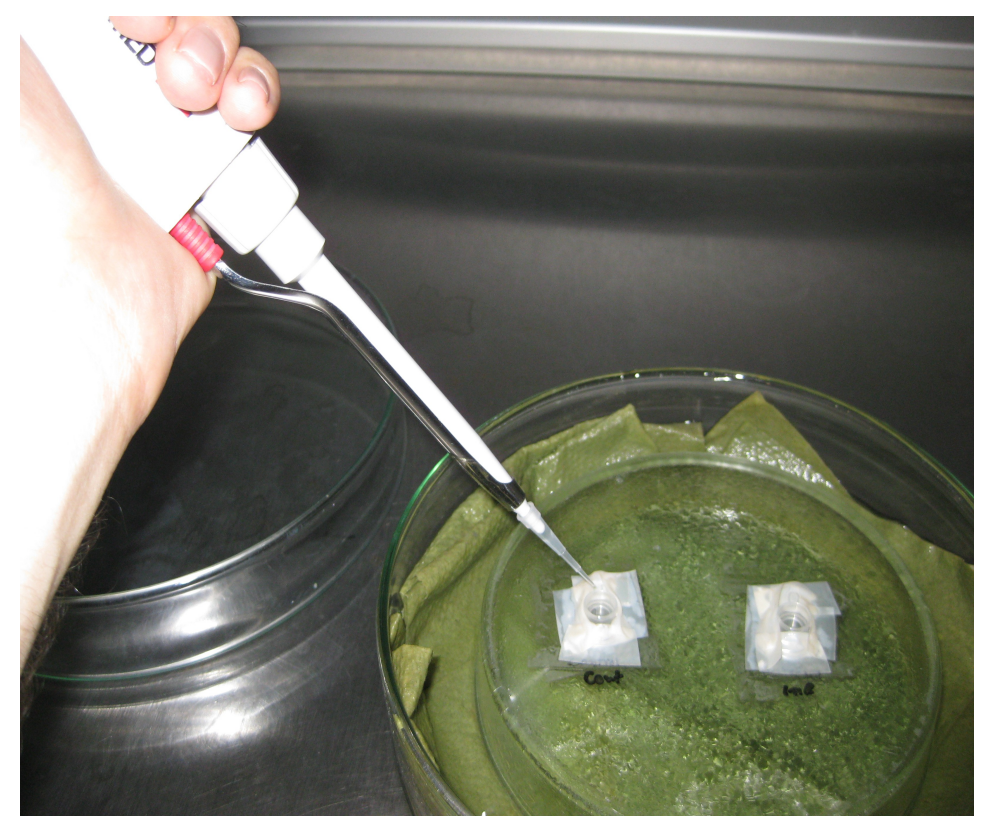

Figure.1. The moister chamber was used in the experiment to prevent the xylem sap from drying.

\section{Inoculum of the bacteria and yeast}

The bacteria A. tumefaciens were taken from stock and plated on Agar plates and were left to grow overnight, then the bacterium was transferred to $50 \mathrm{ml}$ LB medium and left to grow for 30 hours. $2 \mu \mathrm{l}$ of the bacterial culture was diluted by adding it to $1.5 \mathrm{ml}$ SXM medium in sterile $2 \mathrm{ml}$ Eppendorf tubes. This was centrifuged for 2 minutes at $10.000 \mathrm{rpm}$, the supernatant was removed, then the pellet was resuspended in another $1.5 \mathrm{ml} \mathrm{SXM}$ medium, of which $2 \mu$ transferred into $50 \mu$ of xylem sap. The inoculum of the yeast was prepared with the same procedure, using YPD medium instead of LB medium as in the case of the bacteria.

The moisture chamber was sealed with para-film and the xylem sap was incubated at 28 ${ }^{\circ} \mathrm{C}$ for bacteria and $30{ }^{\circ} \mathrm{C}$ for the yeast. 


\section{Plating}

$2 \mu \mathrm{l}$ of the xylem sap with the bacterium or yeast, was added to $100 \mu \mathrm{l}$ sterile water, mixed well and then the whole mixture was spread on one (LB or YPD) Agar plate. Dilutions were made by adding $2 \mu \mathrm{l}$ of the xylem sap with the bacterium to $100 \mu \mathrm{l}$ sterile water and dilutions of $5,25,125,625,3125$ times were made respectively. Four plates were made from each dilution. Plating at time $0 \mathrm{~h}$ and then three additional time points over two days at 12 hours, 24 hours, 48 hours.

\section{Treatments}

$50 \mu \mathrm{l}$ of xylem sap from $V$. longisporum infected oilseed rap plants.

$50 \mu$ l of xylem sap from non infected oilseed rap plants.

\section{Counting of the colonies}

The number of bacterial colonies were counted under binocular at different time points and different dilutions, the number of the bacterial cells in xylem sap was calculated. The number of the yeast cells were counted in the same way.

\section{Statistical analysis and graphs}

For statistical analysis we used the statistical analysis software STATISTICA (StatSoft $\mathrm{GmbH}$, Hamburg, Germany), by using t-test, we tested the signification of the differences between between means at different time points for the growth of microorganism in xylem sap from the infected and non infected plants. For creation of graphs we used Libreoffice package which is a free office applications at: http://www.libreoffice org/ features/calc/ 


\section{Results}

\section{Bioassay on the bacteria Agrobacterium tumefaciens}

\section{The unfiltered xylem sap}

A. tumefaciens was grown in xylem sap extracted from B. napus infected with $V$. longisporum and from healthy plants. Bacterial colonies were counted after 10, 24, 48 hours and then the growth of the bacteria was estimated. The results are shown in Fig. 2. At one time-point $(24 \mathrm{~h})$, the growth was significantly inhibited in the unfiltered xylem sap (containing large molecules) taken from $V$. longisporum infected plants as compared to xylem sap taken from healthy plant.

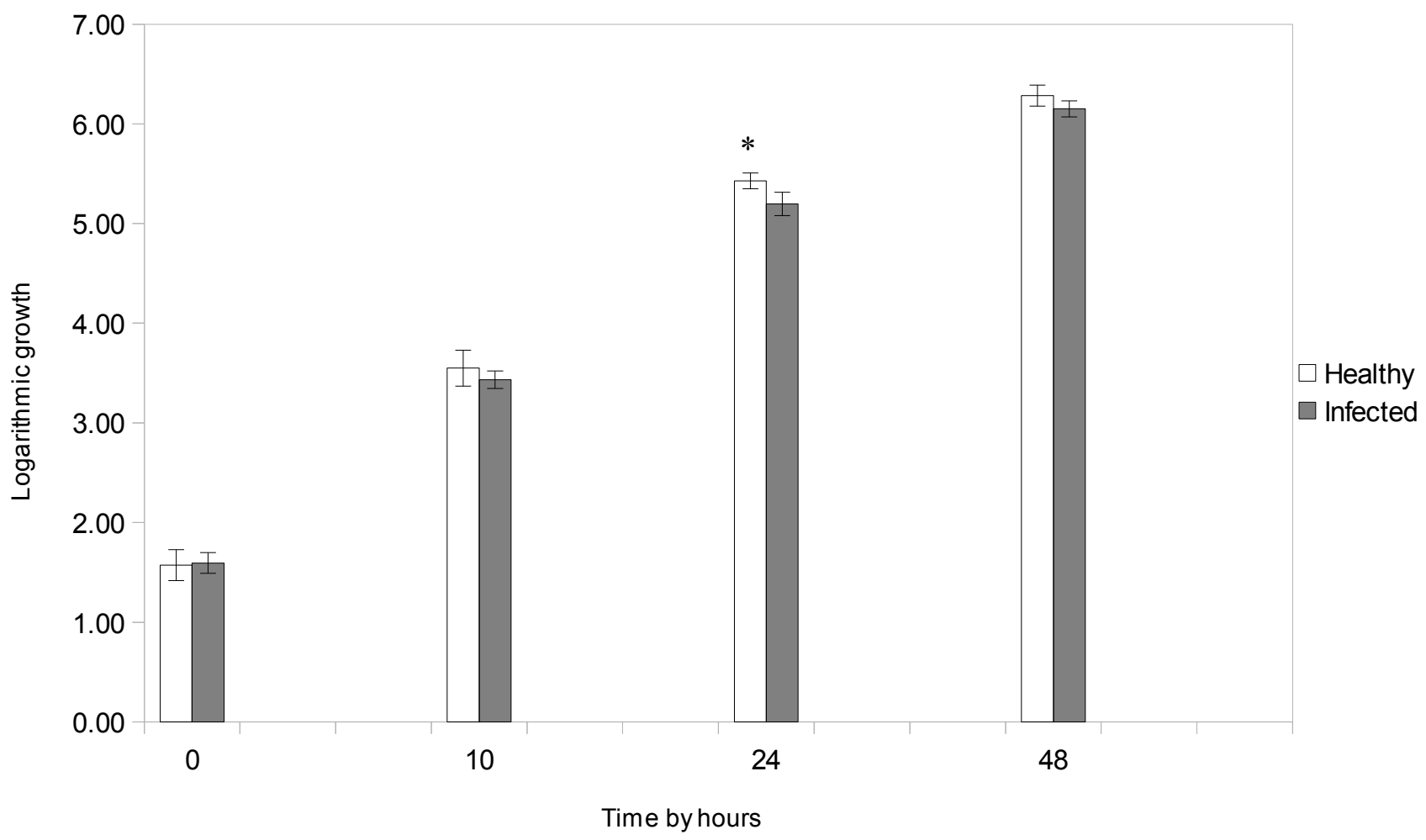

Figure 2. Growth of $A$. tumefaciens in the unfiltered xylem sap from both $V$. longisporum infected and healthy $B$. napus plants. $A$. tumefaciens was grown in unfiltered xylem sap from infected and healthy plants in three different replicates. At the time points indicated, dilutions of the cultures were plated on agar media to determine the cfus. The significance of the differences between the mean was tested by t-test, significant differences are labeled by an asterix. 
The assay was repeated on xylem sap taken from $V$. longisporum infected and uninfected B. napus plants obtained from Christine Druebert from Prof. Dr. Andrea Polle research group (Department of Forest Botany and Tree Physiology, Georg-August University, Göttingen).The results were similar to the previous observation described above. There was an inhibition effect on the growth in the unfiltered xylem sap taken from $V$. longisporum infected plants in comparison with the growth in the unfiltered xylem sap taken from healthy plants as shown in Fig 3.

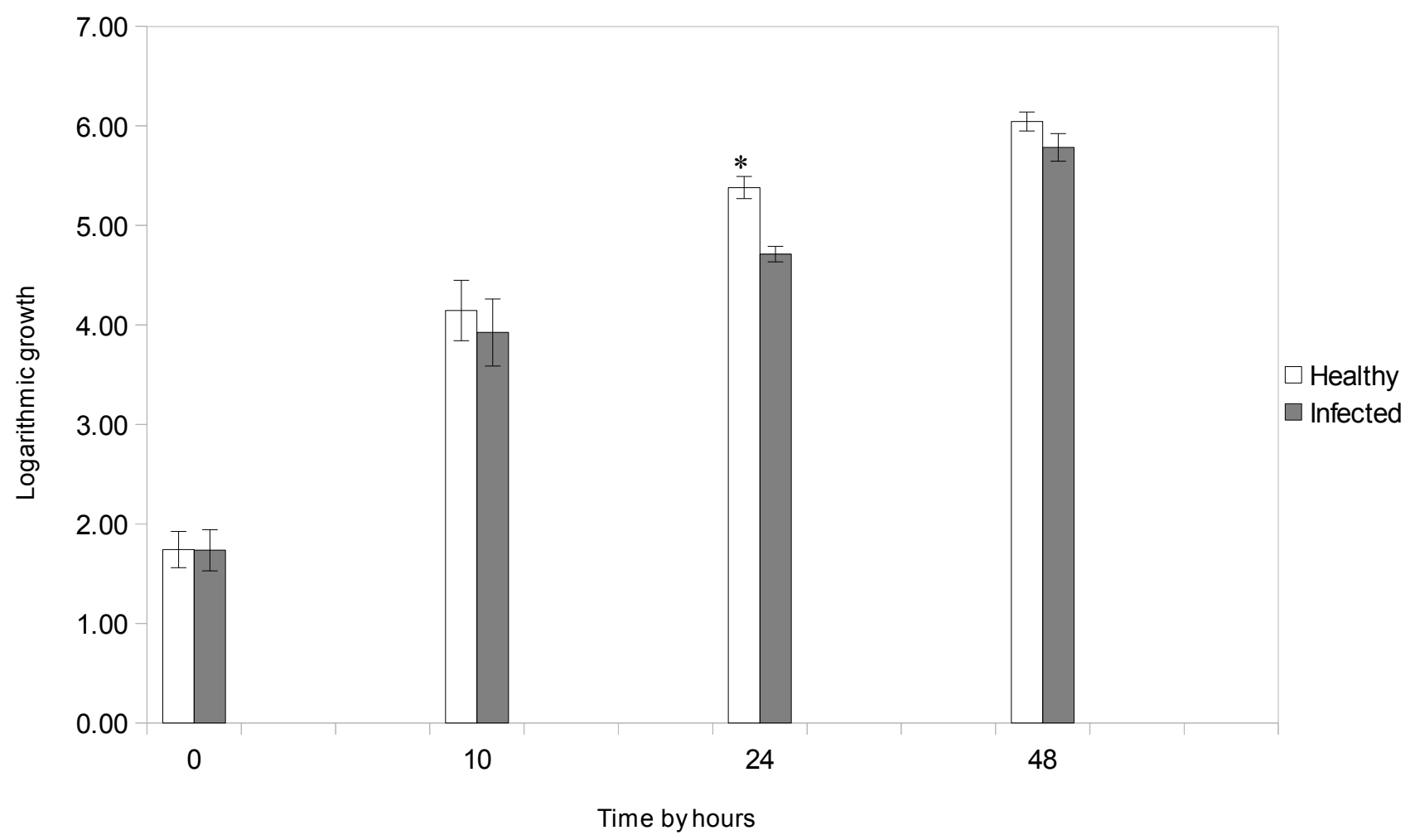

Figure 3. Growth of $A$. tumefaciens in the unfiltered xylem sap from both $V$. longisporum infected and healthy $B$. napus plants (The xylem sap provided by Prof. Dr. Polle research group). The growth of the bacteria A. tumefaciens was inhibited significantly after 24 hours of growing in xylem sap taken from infected plants in comparison to the growth in unfiltered xylem sap taken from healthy plants. 


\section{The macromolecules-filtered xylem sap}

The results of macromolecules-filtered xylem sap provided by $\mathrm{C}$. Druebert are shown in Fig. 4. The inhibition effect on the growth of $A$. tumefaciens was not observed when we used xylem sap, from which macromolecules larger than $3 \mathrm{kDa}$ were removed; the growth was similar in both xylem sap from healthy plant and infected plants.

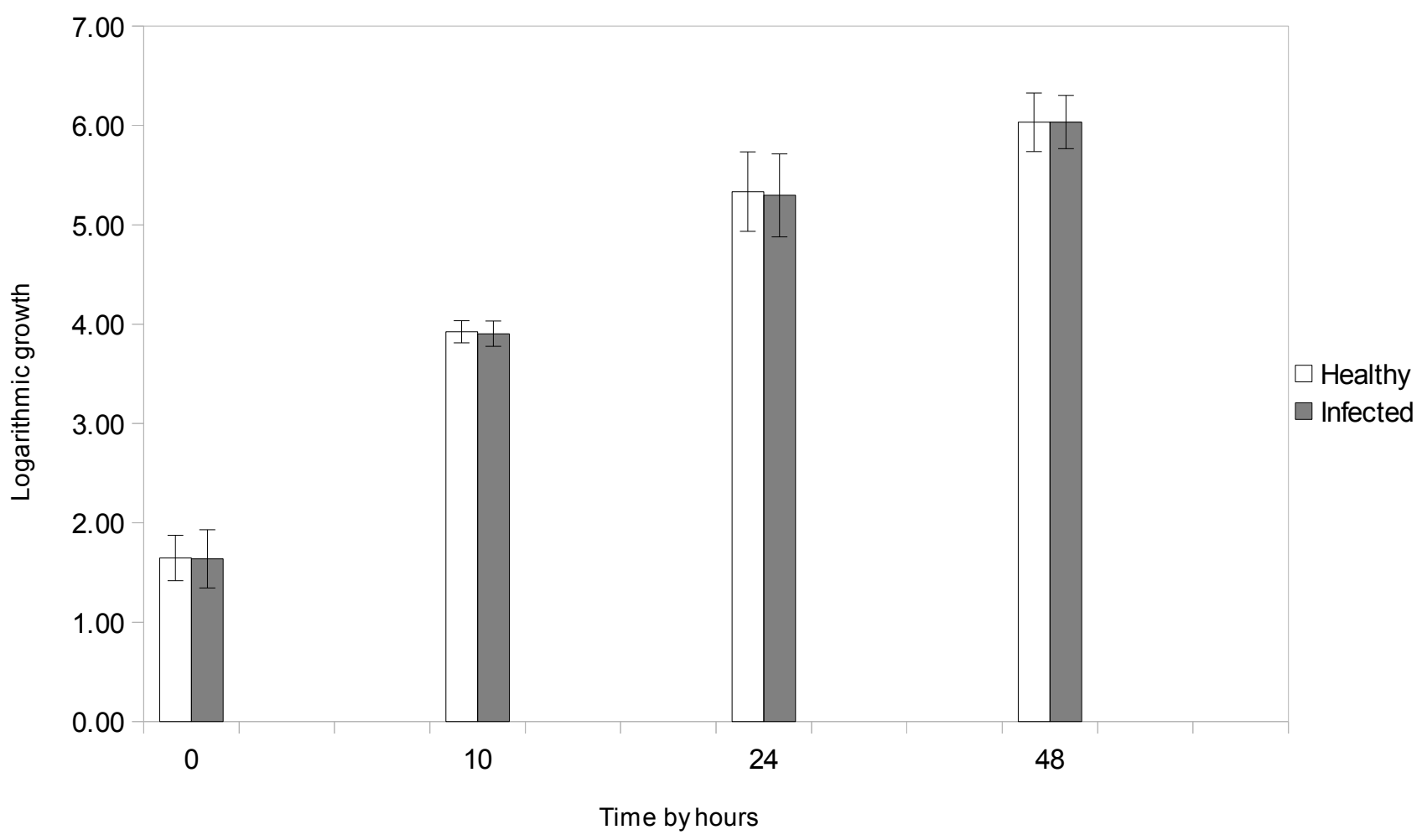

Figure 4. Growth of $A$. tumefaciens in the filtered xylem sap (without macromolecules) from both $V$. longisporum infected and healthy $B$. napus plants(The macromolecules filtered xylem sap was provided by Prof. Dr. Polle research group). No inhibition effect was observed when large molecules were removed from xylem sap by 3000 MWCO membrane filtration.

\section{Bioassay on the yeast Saccharomyces cerevisiae}

\section{The unfiltered xylem sap}

The same assay was conducted on other microorganism, the yeast Saccharomyces cerevisiae by using xylem sap taken from $V$. longisporum- infected and uninfected $B$. napus plants. We used the unfiltered xylem sap. The number of the colonies of Saccharomyces cerevisiae were counted in different time points after 10, 24, 48 hours post inoculation, and then the growth of the yeast was estimated. 
The result showed no significant difference between the growth on xylem sap from $V$. longisporum infected and healthy B. napus plants as shown in Fig. 5.

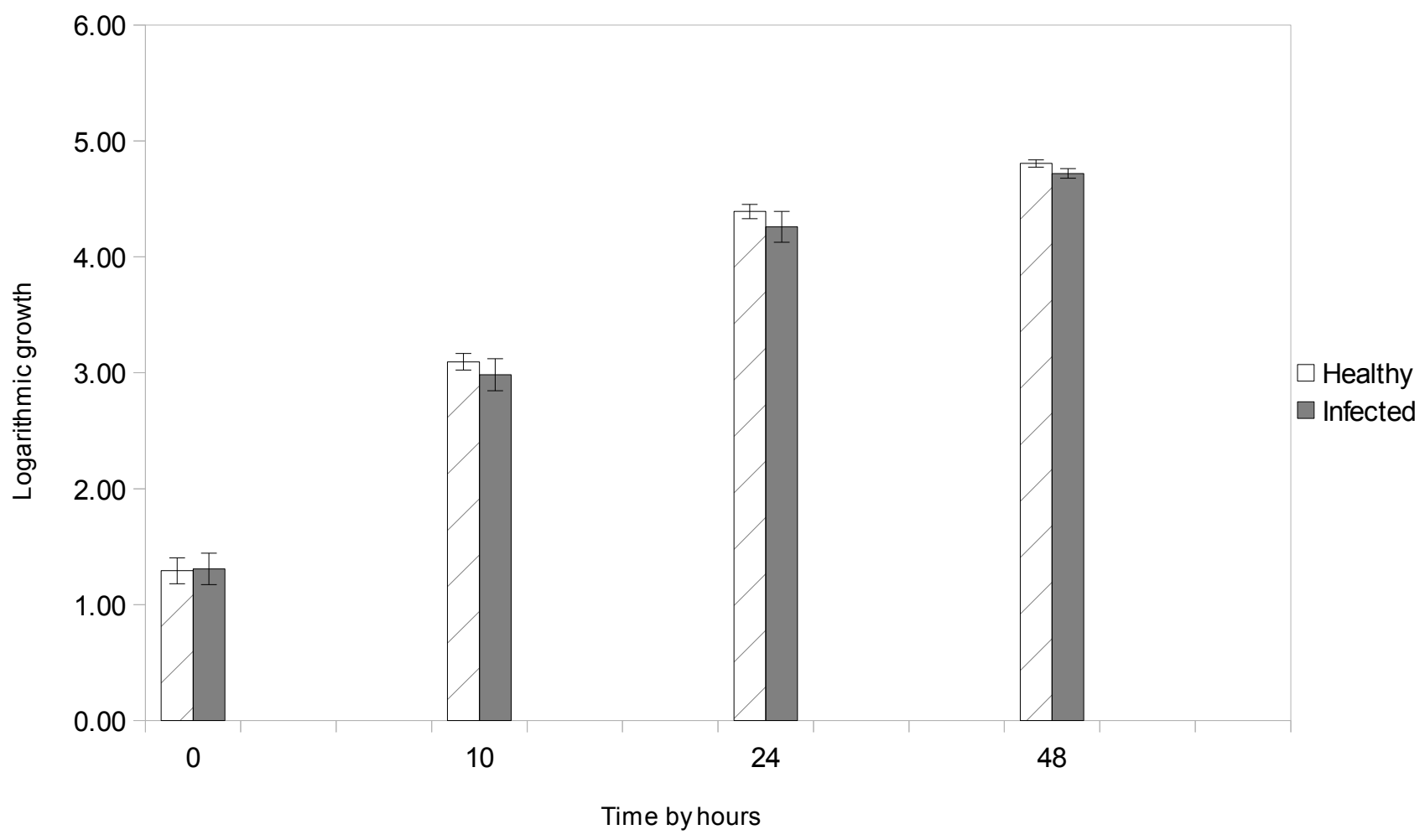

Figure 5. Growth of S. cerevisiae in the unfiltered xylem sap from both $V$. longisporum infected and healthy $B$. napus plants. There was no significant difference in the growth of Saccharomyces cerevisiae when a comparison made between the growth in unfiltered xylem sap taken from infected and healthy plants. 
Furthermore, xylem sap provided by Christine Druebert showed no significant difference between the growth of $S$. cerevisiae in unfiltered xylem sap taken from $V$. longisporum infected and healthy B. napus plants as shown in Fig 6.

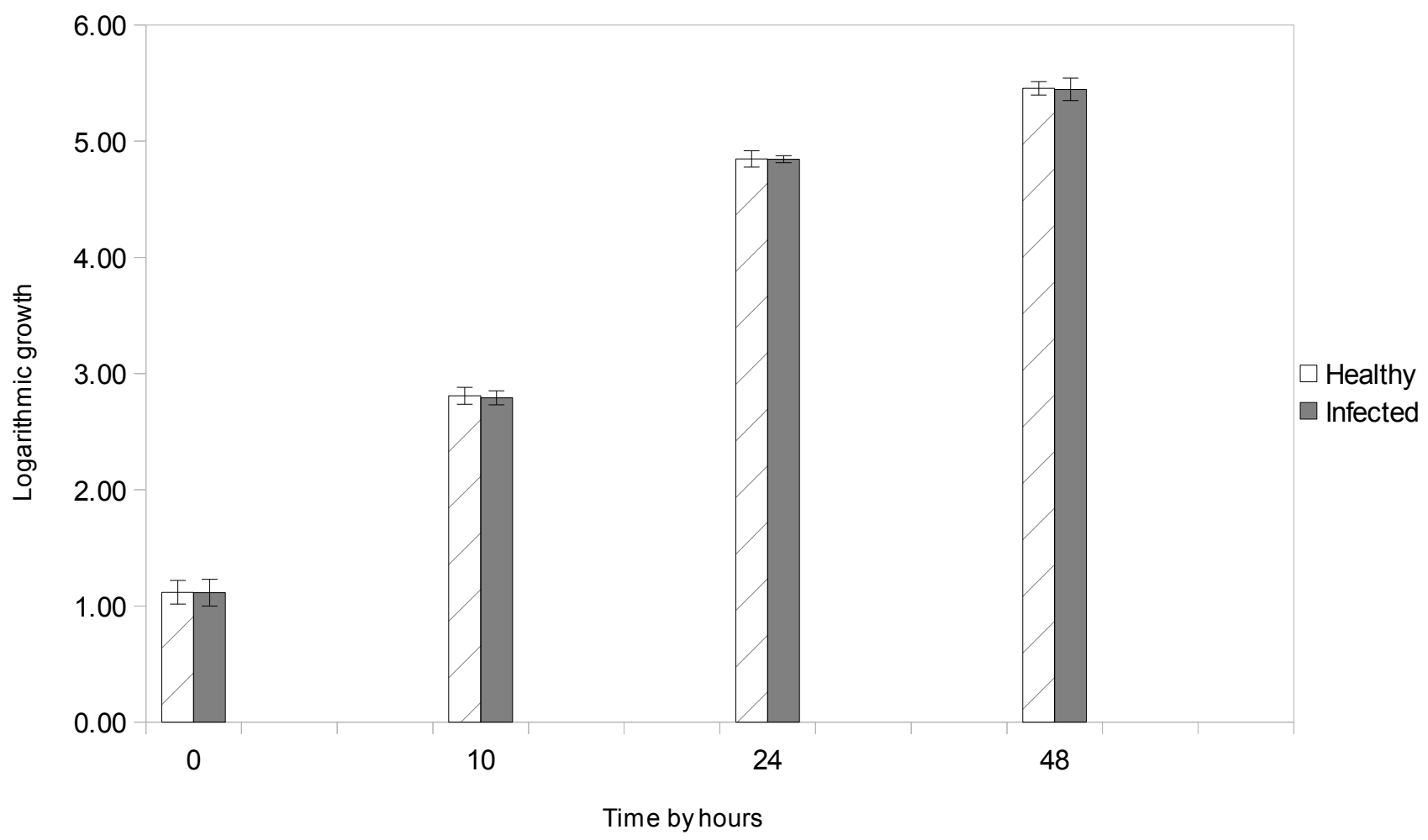

Figure 6. Growth of $S$. cerevisiae in the unfiltered xylem sap from both $V$. longisporum infected and healthy $B$. napus plants (The xylem sap was provided by Prof. Dr. Polle research group). The growth of Saccharomyces cerevisiae showed no differences by growing on unfiltered xylem sap taken from infected and healthy plants. 


\section{The macromolecules-filtered xylem sap}

The effect remained the same when we compared the growth of Saccharomyces cerevisiae in macromolecules filtered xylem sap from $V$. longisporum infected and healthy B. napus plants as shown in Fig 7.

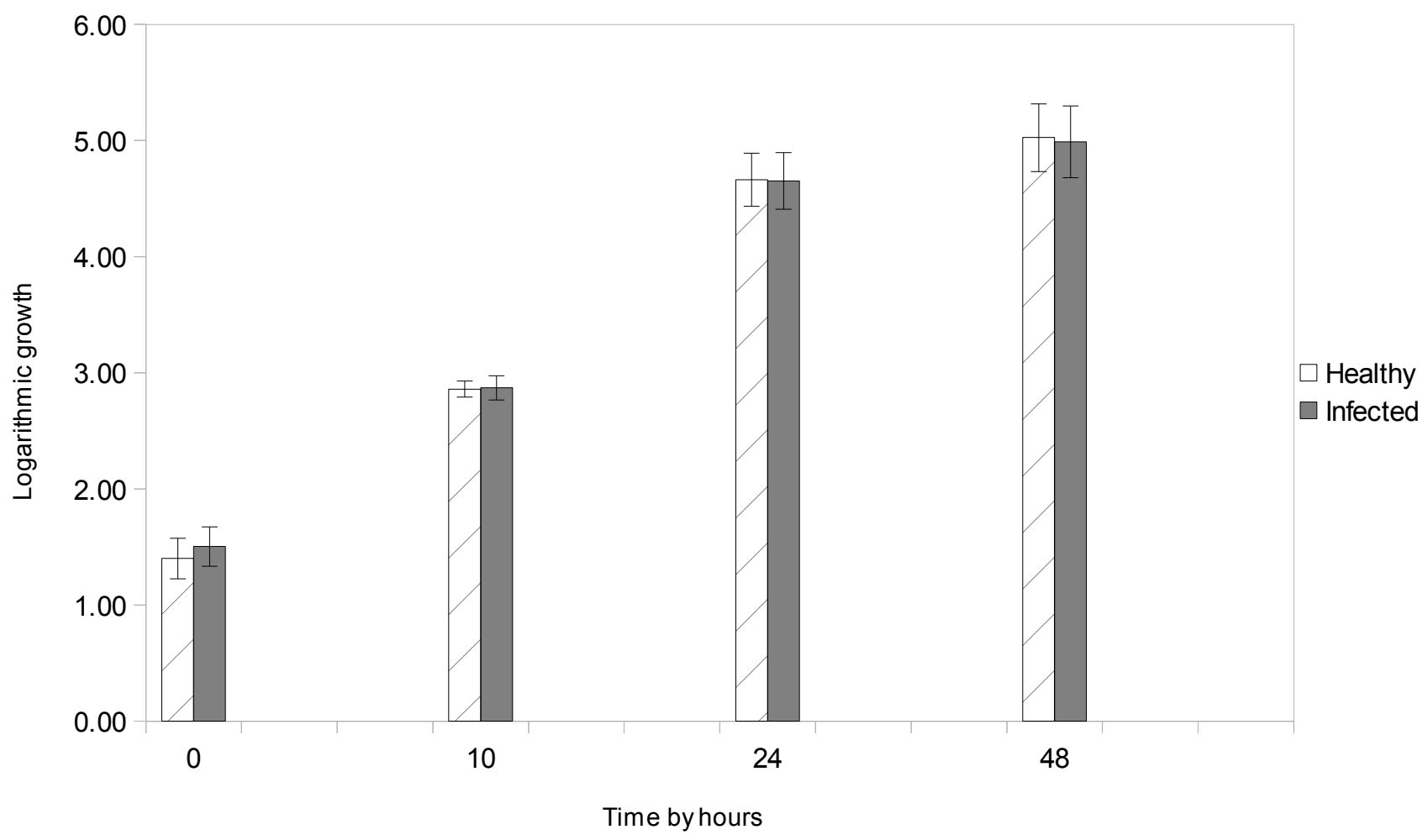

Figure 7. Growth of Saccharomyces cerevisiae in the filtered xylem sap from both infected and healthy plants (The macromolecules filtered xylem sap was provided by Prof. Dr. Polle research group). The growth of Saccharomyces cerevisiae showed no differences by growing in xylem sap taken from healthy plants in comparison the growth in the filtered xylem sap taken from $V$. longisporum infected $B$. napus plants.

As a result, there was no effect of $V$. longisporum infection on the growth of $S$. cerevisiae in both filtered or unfiltered xylem sap taken from B. napus plants. 


\section{Discussion}

$V$. longisporum infects oilseed rape $B$. napus and stays restricted to the xylem vessels during most of its biotrophic phase of its life cycle the invading pathogen (Eynck et al., 2007). Xylem sap is the environment in which biochemical exchange between the plant microbe takes place (Ratzinger, 2008). Changes in the composition of the xylem sap can be observed in the level of phytohormones as a part of the plant mechanism of defense (Ratzinger et al., 2009) Small molecules were found that were specific for $V$. longisporum infection on B. napus (Ratzinger, 2008). The pathogen induced plant defense involves modification of metabolic compounds and accumulation of phenolic compounds (Eynck et al., 2009; Obermeier et al., 2013). Despite of these changes, V. Iongisporum infection has no effect on the nutrients in the xylem sap (Floerl et al., 2008), and the yeast $S$. cerevisiae. In the case of $A$. tumefaciens, the growth of the bacterium was inhibited in the unfiltered xylem from infected plants in comparison to growth in unfiltered xylem from healthy plants. The inhibition effect was significant after 24 hours after the inoculation in the xylem as shown in Fig. 2 and Fig. 3 This effect was not observed when macromolecules were removed from the xylem sap (Fig. 4). This provides evidence that the inhibition effect, which was observed, is due to macromolecules present in xylem. Xylem sap contains naturally several proteins including chitinases and the disease resistance response protein (PR-1), in addition to oxidative stress proteins of peroxidases (Kehr et al., 2005). V. longisporum induces defense mechanism in the plant which result in expressing large molecules secreted in xylem sap of $B$. napus. There is evidence that these macromolecules are produced by plants to inhibit the fungus (Singh et al., 2012).The proteins secreted in the xylem sap include several upregulated proteins like endochitinase, peroxidase, PR-4 protein and $\beta$-1,3-glucanase. (Floerl et al., 2008), previous studies showed PR4-protein is induced in response to Pseudomonas syringae in Brassica rapa (Park and Cho, 2003; Ryang et al., 2002). This protein possesses anti-fungal activity in different crops in several studies (Caporale et al., 2004; Zhu et al., 2006) 
Therefore the inhibition effect on A. tumefaciens is attributed to these non-specific proteins, especially PR-4 protein which is known to be produced in Brassica against $P$. syringae, which is also a gram negative bacterium as well as $A$. tumefaciens (Ryang et al., 2002).

The inhibition effect of the $V$. longisporum on the growth of $S$. serveiciae was limited or negligible as we see in Fig. 5, Fig. 6 and Fig. 7. This can be explained as the yeast was not strongly inhibited by the proteins which are produced in response to $\mathrm{V}$. longisporum.

The result of this assay indicates that small molecules present in the xylem sap of infected $B$. napus have no direct effect on the growth of the studied microorganisms. The infection of $B$. napus has a limited effect on the yeast. The reason can be that these compounds are very specific for the pathogen $V$. longisporum and have a limited effect on other microorganisms. Another possibility is that these phytoalexins were subjected to detoxification by the fungus and have lost ability to inhibit these microorganisms as a result, as it is known that other plant pathogens are able to detoxify or redirect the synthetic pathway of Brassica phytoalexins (Pedras and Ahiahonu, 2005). The stability of secondary metabolites is also a critical factor in the effect of these compounds (Pedras, 2011), especially considering that in this assay, these compounds were exposed to aerobic conditions and they probably underwent oxidation. An optimization of this assay insuring the stability of small molecules is still required. However studying the effect of these de novo synthesized compounds is not straightforward. 


\section{References}

Caporale, C., Di Berardino, I., Leonardi, L., Bertini, L., Cascone, A., Buonocore, V., Caruso, C., 2004. Wheat pathogenesis-related proteins of class 4 have ribonuclease activity. Febs Lett. 575, 71-76.

Darvill, A.G., Albersheim, P., 1984. Phytoalexins and their Elicitors-A Defense Against Microbial Infection in Plants. Annu. Rev. Plant Physiol. 35, 243-275.

Dunker, S., Keunecke, H., Steinbach, P., Von Tiedemann, A., 2008. Impact of Verticillium longisporum on Yield and Morphology of Winter Oilseed Rape (Brassica napus) in Relation to Systemic Spread in the Plant. J. Phytopathol. 156, 698-707.

Entian, K.-D., Schuster, T., Hegemann, J.H., Becher, D., Feldmann, H., Güldener, U., Götz, R., Hansen, M., Hollenberg, C.P., Jansen, G., Kramer, W., Klein, S., Kötter, P., Kricke, J., Launhardt, H., Mannhaupt, G., Maierl, A., Meyer, P., Mewes, W., Munder, T., Niedenthal, R.K., Rad, M.R., Röhmer, A., Römer, A., Rose, M., Schäfer, B., Siegler, M.L., Vetter, J., Wilhelm, N., Wolf, K., Zimmermann, F.K., Zollner, A., Hinnen, A., 1999. Functional analysis of 150 deletion mutants in Saccharomyces cerevisiae by a systematic approach. Mol. Gen. Genet. Mgg 262, 683-702.

Eynck, C., Koopmann, B., Grunewaldt-Stoecker, G., Karlovsky, P., Tiedemann, A. von, 2007. Differential interactions of Verticillium longisporum and V. dahliae with Brassica napus detected with molecular and histological techniques. Eur. J. Plant Pathol. 118, 259274.

Eynck, C., Koopmann, B., Karlovsky, P., von Tiedemann, A., 2009. Internal Resistance in Winter Oilseed Rape Inhibits Systemic Spread of the Vascular Pathogen Verticillium longisporum. Phytopathology 99, 802-811.

Floerl, S., Druebert, C., Majcherczyk, A., Karlovsky, P., Kües, U., Polle, A., 2008. Defence reactions in the apoplastic proteome of oilseed rape (Brassica napus var. napus) attenuate Verticillium longisporum growth but not disease symptoms. Bmc Plant Biol. 8, 129. 
Fradin, E.F., Thomma, B.P.H.J., 2006. Physiology and molecular aspects of Verticillium wilt diseases caused by V. dahliae and V. albo-atrum. Mol. Plant Pathol. 7, 71-86.

Heale, J.B., Karapapa, V.K., 1999. The Verticillium threat to canada's major oilseed crop: canola. Can. J. Plant Pathol. 21, 1-7.

Inderbitzin, P., Davis, R.M., Bostock, R.M., Subbarao, K.V., 2011. The Ascomycete Verticillium longisporum Is a Hybrid and a Plant Pathogen with an Expanded Host Range. Plos One 6, e18260.

Johansson, A., Goud, J.-K.C., Dixelius, C., 2006. Plant Host Range of Verticillium longisporum and Microsclerotia Density in Swedish Soils. Eur. J. Plant Pathol. 114, 139149.

Karapapa, V.K., Bainbridge, B.W., Heale, J.B., 1997. Morphological and molecular characterization of Verticillium longisporum comb, nov., pathogenic to oilseed rape. Mycol. Res. 101, 1281-1294.

Kehr, J., Buhtz, A., Giavalisco, P., 2005. Analysis of xylem sap proteins from Brassica napus. Bmc Plant Biol. 5, 11.

López-Millán, A.F., Morales, F., Andaluz, S., Gogorcena, Y., Abadía, A., Rivas, J.D.L., Abadía, J., 2000. Responses of Sugar Beet Roots to Iron Deficiency. Changes in Carbon Assimilation and Oxygen Use. Plant Physiol. 124, 885-898.

Neumann, M.J., Dobinson, K.F., 2003. Sequence tag analysis of gene expression during pathogenic growth and microsclerotia development in the vascular wilt pathogen Verticillium dahliae. Fungal Genet. Biol. Fg B 38, 54-62.

Obermeier, C., Hossain, M.A., Snowdon, R., Knüfer, J., Tiedemann, A. von, Friedt, W., 2013. Genetic analysis of phenylpropanoid metabolites associated with resistance against Verticillium longisporum in Brassica napus. Mol. Breed. 1-15.

Park, Y., Cho, T., 2003. Isolation and characterization of methyl jasmonate-inducible genes in chinese cabbage. Korean J. Biol. Sci. 7, 337-343. 
Pedras, M.S.C., Ahiahonu, P.W.K., 2005. Metabolism and detoxification of phytoalexins and analogs by phytopathogenic fungi. Phytochemistry $66,391-411$.

Pedras, M.S.C., Yaya, E.E., 2010. Phytoalexins from Brassicaceae: news from the front. Phytochemistry 71, 1191-7.

Pedras, M.S.C., Yaya, E.E., Glawischnig, E., 2011. The phytoalexins from cultivated and wild crucifers: chemistry and biology. Nat. Prod. Rep. 28, 1381-405.

Pegg, G.F., Brady, B.L., 2002. Verticillium wilts 2002 pp. UK.

Ratzinger, A., 2008. Development and application of LC-MS-based differential metabolic profiling in plant systems. Georg-August-Universität Göttingen, Göttingen,Germany.

Ratzinger, A., Riediger, N., von Tiedemann, A., Karlovsky, P., 2009. Salicylic acid and salicylic acid glucoside in xylem sap of Brassica napus infected with Verticillium longisporum. J. Plant Res. 122, 571-579.

Ryang, S.H., Chung, S.Y., Lee, S.H., Cha, J.S., Yong Kim, H., Cho, T.J., 2002. Isolation of pathogen-induced Chinese cabbage genes by subtractive hybridization employing selective adaptor ligation. Biochem. Biophys. Res. Commun. 299, 352-359.

Siebold, M., Tiedemann, A. von, 2012. Potential effects of global warming on oilseed rape pathogens in Northern Germany. Fungal Ecol. 5, 62-72.

Singh, S., Braus-Stromeyer, S.A., Timpner, C., Valerius, O., von Tiedemann, A., Karlovsky, P., Druebert, C., Polle, A., Braus, G.H., 2012. The plant host Brassica napus induces in the pathogen Verticillium longisporum the expression of functional catalase peroxidase which is required for the late phase of disease. Mol. Plant-Microbe Interactions Mpmi 25, 569-581.

Tilsner, J., Kassner, N., Struck, C., Lohaus, G., 2005. Amino acid contents and transport in oilseed rape (Brassica napus L.) under different nitrogen conditions. Planta 221, 328338. 
VanEtten, H., Mansfield, J., Bailey, J., Farmer, E., 1994. Two Classes of Plant Antibiotics: Phytoalexins versus "Phytoanticipins". Plant Cell 6, 1191-1192.

Zeise, K., Tiedemann, A. von, 2001. Morphological and Physiological Differentiation among Vegetative Compatibility Groups of Verticillium dahliae in Relation to $V$. longisporum. J. Phytopathol. 149, 469-475.

Zeise, K., Von Tiedemann, A., 2002. Host Specialization among Vegetative Compatibility Groups of Verticillium dahliae in Relation to Verticillium longisporum. J. Phytopathol. 150, 112-119.

Zhou, L., Hu, Q., Johansson, A., Dixelius, C., 2006. Verticillium longisporum and V. dahliae: infection and disease in Brassica napus. Plant Pathol. 55, 137-144.

Zhu, T., Song, F., Zheng, Z., 2006. Molecular Characterization of the Rice Pathogenesisrelated Protein, OsPR-4b, and Its Antifungal Activity Against Rhizoctonia solani. J. Phytopathol. 154, 378-384. 


\title{
Chapter 4: Comparative metabolic profiling of wild type and polyketide synthase silenced strain of Verticillium longisporum
}

\author{
Husam Ibrahem Aroud, Malte Beinhoff and Petr Karlovsky \\ Molecular Phytopathology and Mycotoxin Research Unit, Department of Crop Sciences, University of \\ Göttingen, Grisebachstrasse 6, 37077 Göttingen, Germany
}

\section{Abstract}

Pathogenic fungi produce a wide range of secondary metabolites, of which polyketides are the most important class of fungal secondary metabolites. They can be used by fungi as phytotoxins in their interactions with the host plant and are considered to be virulence factors in many pathogens. In the previous work, the gene VI-PKS-1, putatively encoding a polyketide synthase (PKS), was identified in the pathogenic fungus Verticillium longisporum and showed similarity to other fungal PKS genes of the subclass wA-type of the fungal polyketide synthase genes. A role of this gene in the interaction between $V$. longisporum with host plant oilseed rape Brassica napus was hypothesized. In this work, we report the metabolic profile of the wild type of $V$. Iongisporum compared to the gene knock down mutant $V$. longisporum PKS-1 when both are grown on potato dextrose broth media. A reddish color was observed in the media after 30 days of inoculation when the wild type grew on it. We conducted comparative metabolic profiling of extracts of the culture supernatant of the both wild type and mutant by using diode array spectrometry (DAD) combined with time of flight (TOF-MS) mass spectrometry detection. The results show that there are 346 marker candidates strongly reduced in the supernatant of the mutant VI-PKS-1. We selected several candidates from these compounds and compared their masses with the known compounds of same masses. Here we report their masses and corresponding candidate metabolites which may be affected by silencing the VI-PKS-1 gene.

Key words: Secondary metabolites, Verticillium longisporum, polyketide synthase (PKS), metabolic profiling. 


\section{Introduction}

Metabolomics or metabolic fingerprinting using MS or NMR analysis is an efficient bioanalytical tool which is widely used in different fields including pharmaceutical, medical, environmental, and agricultural researches (Dunn and Hankemeier, 2013). By analyzing crude extracts of fungal cultures by means of HPLC-MS, it was possible to distinguish the fungal species of Penicillium from other species by creating a simple library of metabolic profiles (Smedsgaard and Frisvad, 1996). Fungal metabolomics is used as a chemotaxonomic approach to complement molecular approaches in the identification and characterization of fungal isolates (Aliferis et al., 2013). Metabolomic approaches in fungi are also used for detection and identification of new compounds resulting from activating silent genes through molecular approaches (Bertrand et al., 2013). Filamentous fungi produce an extensive array of secondary metabolites with a wide range of bio-activities. In plant/pathogen system, secondary metabolites produced by pathogenic fungi can be pathogenicity or virulence factors (Stergiopoulos et al., 2013; Tsuge et al., 2013). Among fungal secondary metabolites, polyketides comprise a large class of important compounds with wide and varied activities and functions among them. Some of them are well known mycotoxins like the aflatoxin B1, which is produced by particular Aspergillus species and considered to be a fierce environmental carcinogen (Watanabe et al., 1996). Some other polyketides cause pigmentation, like naphthopyrone compound YWA1, which is yellow colored and an intermediate asexual spore pigment of Aspergillus nidulans (Watanabe et al., 1999). Multifunctional enzymes are responsible for the bio-synthesis of polyketides in plants, bacteria and fungi. These enzymes are called polyketide synthases (PKSs) and can be grouped into three types (type I, type II, and type III). The type I of these enzymes are responsible for the bio-syntheses of fungal and bacterial polyketides characterized as multifunctional proteins with a similar domain structure to those which are responsible for synthesizing fatty acids in eukariotics (Hopwood and Sherman, 1990; Keller et al., 2005). Despite the wide diversity of their functions and chemical structure, all polyketides are biosynthesized in prokaryotic and eukaryotic organisms in a common pattern based on the assembly of carbon chains in a mechanism similar to that in fatty acid synthesis. The difference to the common process is the complete reduction of $\beta$-carbon which is obligatory in case of the fatty acid synthesis while it is optional in polyketide synthesis (Hopwood and Sherman, 1990). Advances achieved in different fields revealed that fungal 
genes encoding groups of secondary metabolites are clustered (Keller et al., 2005). Fungal secondary metabolites and their encoding genes can be utilized as an effective tool for studying taxonomy, phylogeny, and evolution of fungi (Stadler and Keller, 2008). The first gene encoding for a PKS in fungi, was discovered in the plant pathogenic species Penicillium patulum (Beck et al., 1990).

$V$. Iongisporum is a hemibiotrophic plant pathogen which infects the plants through the root and then colonizes the vascular system and remains restricted to it for most of its life cycle. In the late stage of the infection, it becomes a necrotrophic fungi and produces microsclerotia (Eynck et al., 2007; Karapapa et al., 1997). A PKS gene from the $V$. longisporum isolate VL43 was identified and named VI-PKS-1. The gene showed a high degree of similarity to previously identified PKS genes of the wA-type, which are known to be involved in pathways of the synthesis of pigmentation (Bingle et al., 1999; Beinhoff, 2011). Four mutants were generated by using silencing technique based on the intracellular expression of an HP (Hair Pin) HP cassette a triggering RNAi in the fungus. This technique was able to silence the VI-PKS-1 gene with efficiency up to $90 \%$ (Beinhoff, 2011). Despite this, the study concluded that VI-PKS-1 is not involved in the virulence or pathogencity during its interaction with Brassica napus. Clear morphological and physiological changes were observed in the transformants including increased growth rates or late pigmentation (Beinhoff, 2011). Furthermore, after 16 days a reddish color was observed in the PDA and PDB media when V. longisporum VL43 was grown at $22^{\circ} \mathrm{C}$ in the dark. Similar changes in the color of the media were not observed in the case of VI-PKS-1 (Beinhoff, 2011). We presume that this coloration of the media is due to changes in the fungal metabolism as a result of silencing of VI-PKS-1 gene. 


\section{Aim of the work}

The aim of the study is to examine changes in the metabolic profile of the pathogenic fungus $V$. longisporum in wild type and silenced mutant; both grown on PDB medium, such changes in the metabolic profile can explain the red color observed in the medium.

\section{Authors' contributions}

Husam Ibrahem Aroud carried out the inoculation experiment, and the extraction of the fungal cultures for the first HPLC-MS, conducted the preliminary HPLC-MS analysis and wrote the manuscript. Dr. Kirstin Feussner (Department of Plant Biochemistry of the Albrecht-von-Haller Institute for Plant Sciences; University of Göttingen) carried out the second step of the analysis using HPLC with high-resolution MS detection, recorded their UV absorption spectra and wrote the corresponding part of the Materials and Methods section. Malte Beinhoff provided the VI-PKS -1 RNAi silenced strain of V. longisporum which was described by him (Beinhoff, 2011). Petr Karlovsky designed and supervised the study and corrected the manuscript. 


\section{Materials and Methods}

\section{Preparation of fungal cultures}

\section{Fungal isolates}

V. longisporum wild type, VL43 strain which was isolated from oilseed rape plants in northern Germany as described (Zeise and Tiedemann, 2001) was obtained from the Department of Crop Sciences, Section Plant Pathology and Crop Protection, (University of Göttingen, Göttingen, Germany). The spores were stored under $\left(-80^{\circ} \mathrm{C}\right)$.

V. longisporum-PKS-1 mutants,(VI-PKS-1) were constructed as described in the $\mathrm{PhD}$ thesis of Malte Beinhoff (Beinhoff, 2011), and were provided by him (Molecular Phytopatholgy and Mycotoxin Research unit, Department of Crop Sciences, University of Göttingen, Göttingen, Germany.

\section{PDB medium}

PDB was prepared by adding $25.6 \mathrm{~g}$ dry medium to 1 liter of distilled water, the liquid medium was distributed on $300 \mathrm{ml}$ Erlenmeyer flasks by adding $50 \mathrm{ml}$ to each flask, the medium contained in the flasks were autoclaved for 15 minutes under $118{ }^{\circ} \mathrm{C}$. PDB medium was provided commercially by Roth chemicals (Carl Roth $\mathrm{GmbH}$ )+ Co, Karlsruhe, Germany).

\section{Inoculation of PDB media and preparation of fungal cultures}

Fifty $\mathrm{ml}$ of PDB medium contained in flasks were inoculated under sterilized conditions with $1 \times 10^{4}$ spore/ml spore suspension from $V$. longisporum wild type, and VL-PKS-1 m 9 mutant type. The inoculated fungi were left to grow for $30 \mathrm{dpi}$, at room temperature $22^{\circ} \mathrm{C}$, without shaking in the dark. 


\section{Extraction of the cultures}

The mycelia were separated from the supernatant by centrifuging in falcon tubes at 4000 rpm. $10 \mathrm{ml}$ of the supernatant was defatted by adding the same volume of cyclo-hexane and shaking thoroughly for two hours, then the mixture was centrifuged at $4000 \mathrm{rpm}$ for ten minutes to separate the organic phase from the supernatant, the cyclo-hexane phase was removed and discarded.

$10 \mathrm{ml}$ of the supernatant was extracted with 1:1 volume using ethyl acetate, the organic phase was collected. The supernatant was extracted for the second time. The resulting 20 $\mathrm{ml}$ of ethyl acetate extraction was dried completely by speed vac at $30^{\circ} \mathrm{C}$.

\section{Preparation of the samples for HPLC}

The dried ethyl acetate extraction was dissolved with $2 \mathrm{ml}$ of 1:1 volume: methanol/ water. $200 \mu \mathrm{l}$ of the dissolved extraction were transferred to HPLC vials for injection.

\section{HPLC-MS method}

The initial comparative metabolite profiling was conducting using HPLC system. The samples were injected automatically into the system using an autosampler (ProStar 410, from Varian, Darmstadt, Germany), the required pressure was provided by binary pump system (ProStar 210, Varian), gases were removed from the system using a degassing machine (Degassit, MetaChem Technologies) and the column was kept under desired temperature by an oven (Jetstream 2 plus, Techlab, Germany). The HPLC system was combined with an ion trap mass spectrometer (500- MS, Varian) via electrospray ionization (ESI) source was used. $10 \mu \mathrm{L}$ of the sample was injected into a reversed-phase column Polaris C18-Ether ( $100 \times 2 \mathrm{~mm}$ i.d., $3 \mu \mathrm{m}$ particle size, Varian) with a compatible guard column (MetaGuard Polaris C18-Ether, Varian) kept at $40{ }^{\circ} \mathrm{C}$. The mobile phase consisted of a binary gradient of $7 \mathrm{mM}$ acetic acid in $95 \%$ water : $5 \%$ acetonitrile $(A)$ and $7 \mathrm{mM}$ acetic acid in methanol (B) (0 - 5 min $90 \%$ A : $10 \%$ B, $5.0-30.0$ min from $90 \%$ A : $10 \%$ B to $2 \%$ $A$ : $98 \%$ B, $30-38 \min 2 \%$ A : $98 \%$ B, followed by washing and re-equilibration steps at a flow rate of $0.2 \mathrm{ml} / \mathrm{min}$ ). The ESI source was operated either with a negative or a positive spray polarity, the parameters were set as follows (negative/ positive): needle voltage $-3500 \mathrm{~V} /+5000 \mathrm{~V}$, shield voltage $-600 \mathrm{~V} /+600 \mathrm{~V}$, capillary voltage $-/+50 \mathrm{~V}$ drying gas 
(nitrogen) 20 psi (138 kPa) at $250{ }^{\circ} \mathrm{C}$ and nebulizing gas (air) 50 psi (345 kPa). The MS analyzer was operated in full scan mode, mass range $\mathrm{m} / \mathrm{z} 100-800$, scan speed $5000 \mathrm{Da} /$ sec, 3 scans averaged, multiplier voltage set to $1385 \mathrm{~V}$.

\section{Metabolite fingerprinting analysis}

Three biological replicates of both the supernatant of the wild type as well as the pks9 mutant supernatant were extracted and analyzed for metabolic fingerprinting as described by (Floerl et al., 2012). Additionally the total wavelength chromatogram (TWC) from 190 $800 \mathrm{~nm}$ were recorded by a DAD. The TWC of the wild type and VL-PKS-1 mutant were compared manually. Eleven signals could be identified, which show decreased UV/VIS signal intensities in the supernatant of the mutant in contrast to wt. For these signals the UVIVIS spectra and the corresponding mass spectra were analyzed. The exact mass information of the TOF-MS derived mass spectra was used for generating a suitable sum formula and to search the KEGG data base (Kyoto Encyclopedia of Genes and Genomes, (http://www.genome.jp/kegg/ligand.html) to get a putative compound identity. 


\section{Results}

PDB media inoculated with wild type of V. longisporum VL43 showed clear reddish coloration after 30 days of inoculation. The VI-PKS-1 mutant medium remained yellow colored with darker yellowish color than the non inoculated media. As shown in Fig. 1, flasks inoculated with $V$. longisporum wild type in the media turned to red $(C)$ in contrast to VI-PKS-1 mutant type9, which showed no coloration (B).This confirms what (Beinhoff, 2011) had observed in his study.

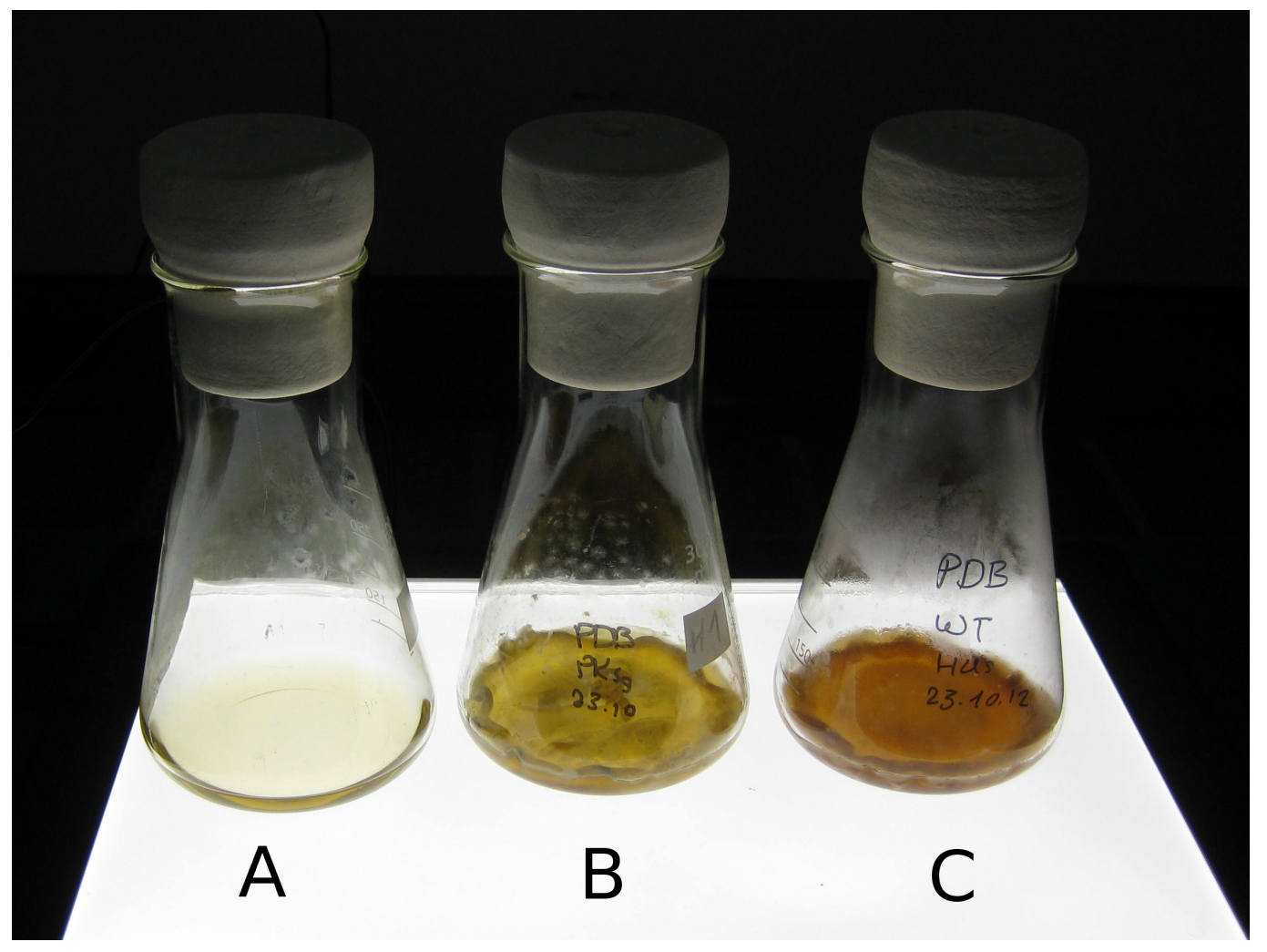

Figure 1: Discoloration of $V$. longisporum cultures in PDB medium

A. Non- inoculated medium, B. The VL-PKS1 mutant, C. V. Iongisporum wild type. Cultures after incubation for 30 days at $23^{\circ} \mathrm{C}$.

The fungal mycelium was removed and the supernatant was extracted with ethyl acetate as described in the Materials and Methods section, then the extracts were subjected to HPLC-MS analysis. The analysis showed differences between the extracted supernatants of $\mathrm{V}$. longisporum and the VI-PKS1 mutant. 
The mass spectra of these peaks revealed number of signals, the major one had a mass to charge ratio of $\mathrm{m} / \mathrm{z} 185.0[\mathrm{M}+\mathrm{H}]^{+}$, and eluted on retention time of 3.7 minutes. Signal 2 , has $\mathrm{m} / \mathrm{z} 122.1[\mathrm{M}+\mathrm{H}]^{+}$eluted on retention time of 10.8 minute, and signal 3 which has the $\mathrm{m} / \mathrm{z} 157.9[\mathrm{M}+\mathrm{H}]^{+}$eluted on retention time of 12.7 minutes.

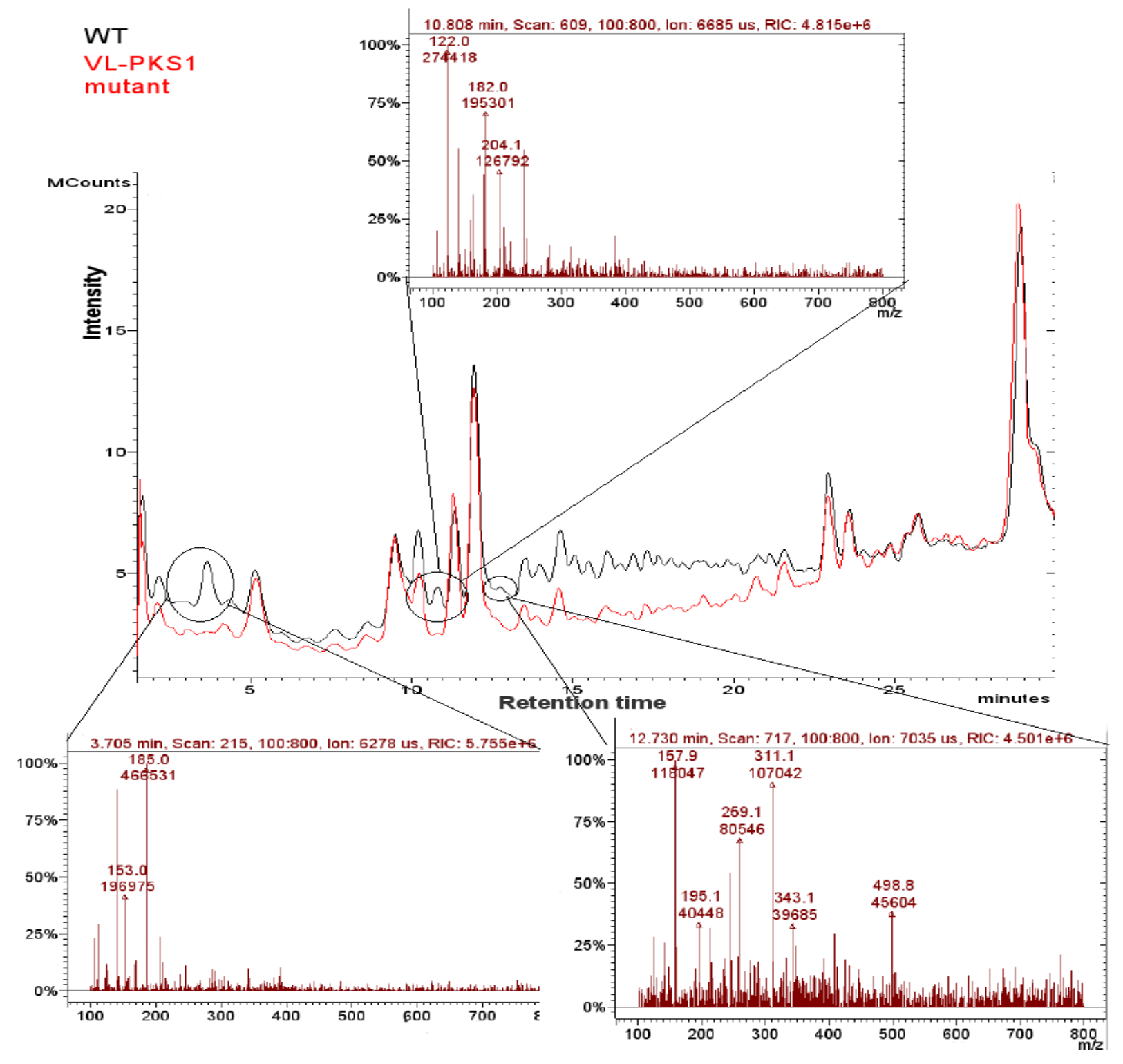

Figure 2: HPLC-MS analysis of culture supernatant of wild type and PKS-1 mutant of $V$. longisporum. Total ion chromatogram of $V$. longisporum wild type (in black), and VI-PKS1 mutant (in red) and the spectra of the peaks circled with black. 
Because of these differences, we carried out an additional UPLC analysis coupled with DAD and full-scan TOF-MS detection The analysis were performed by Dr. Kirstin Feussner in the laboratory of Prof. Feussner (Department of Plant Biochemistry, Brecht-von-Haller Institute for Plant Sciences, Georg-August University, Göttingen). The UVIVIS analysis combined with TOF-MS showed that there were 11 signals, which show different intensity between the V. longisporum wild type and VL-PKS1 mutant type 9 (Fig. 3 a, b and c). 


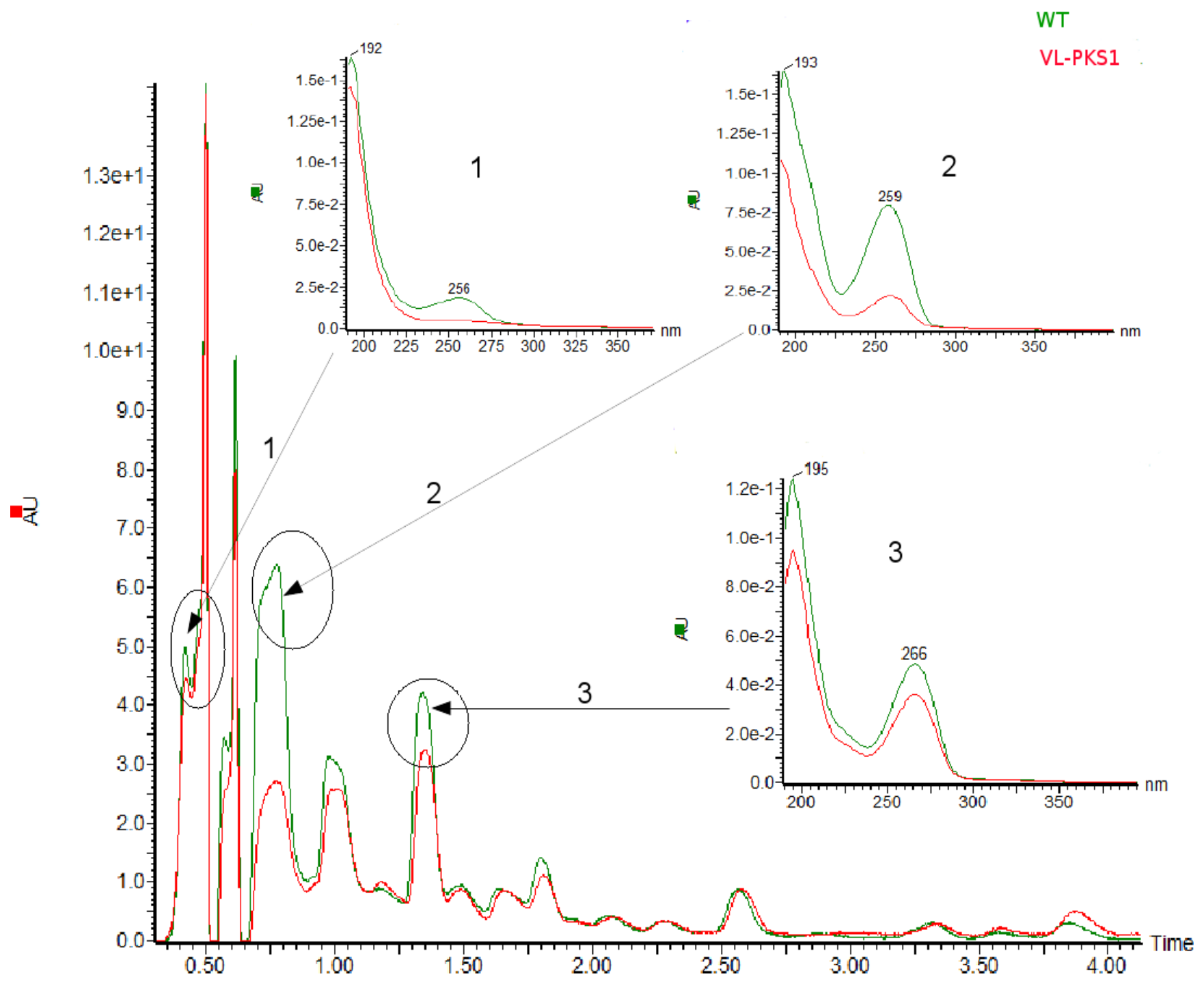

Figure 3a. UV/VIS analysis of supernatant of the $V$. longisporum wild type and PKS-1 mutant The total wavelength chromatogram (190 - $800 \mathrm{~nm}$, section 0 to $4 \mathrm{~min}$ ) is shown for the supernatant of $V$. longisporum wild type (green) and VL-PKS1 mutant (red). The corresponding UVIVIS absorption spectra of the signals 1-3 are shown as inlets. 


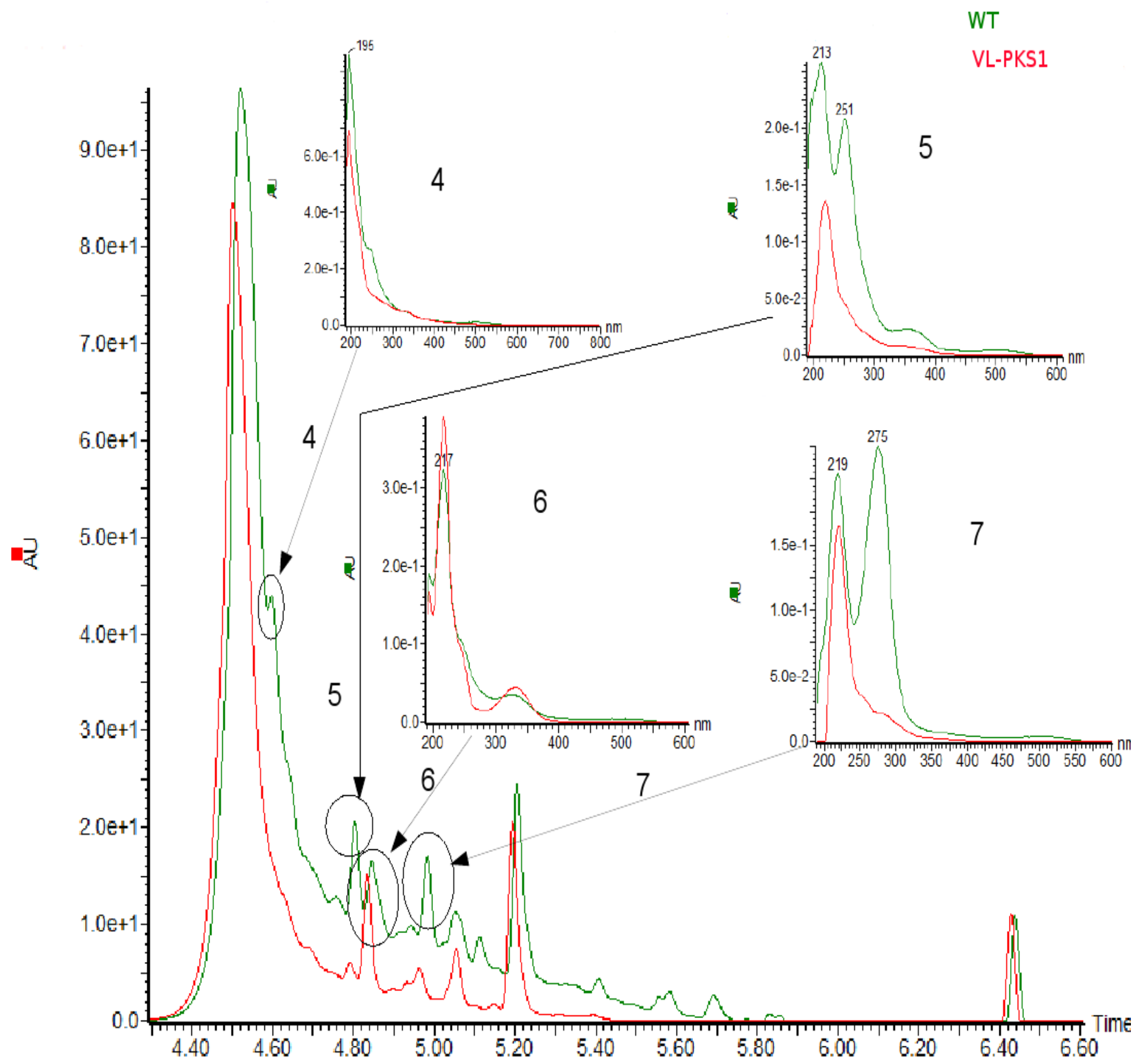

Figure $3 b$. UV/VIS analysis of supernatant of the V. longisporum wild type and VI-PKS-1 mutant

The total wavelength chromatogram (190 - $800 \mathrm{~nm}$, section 4 to $6.6 \mathrm{~min})$ is shown for the supernatant of $V$. longisporum wild type (green) and VL-PKS1 mutant (red). The corresponding UV/VIS absorption spectra of the signals 4-7 are shown as inlets. 


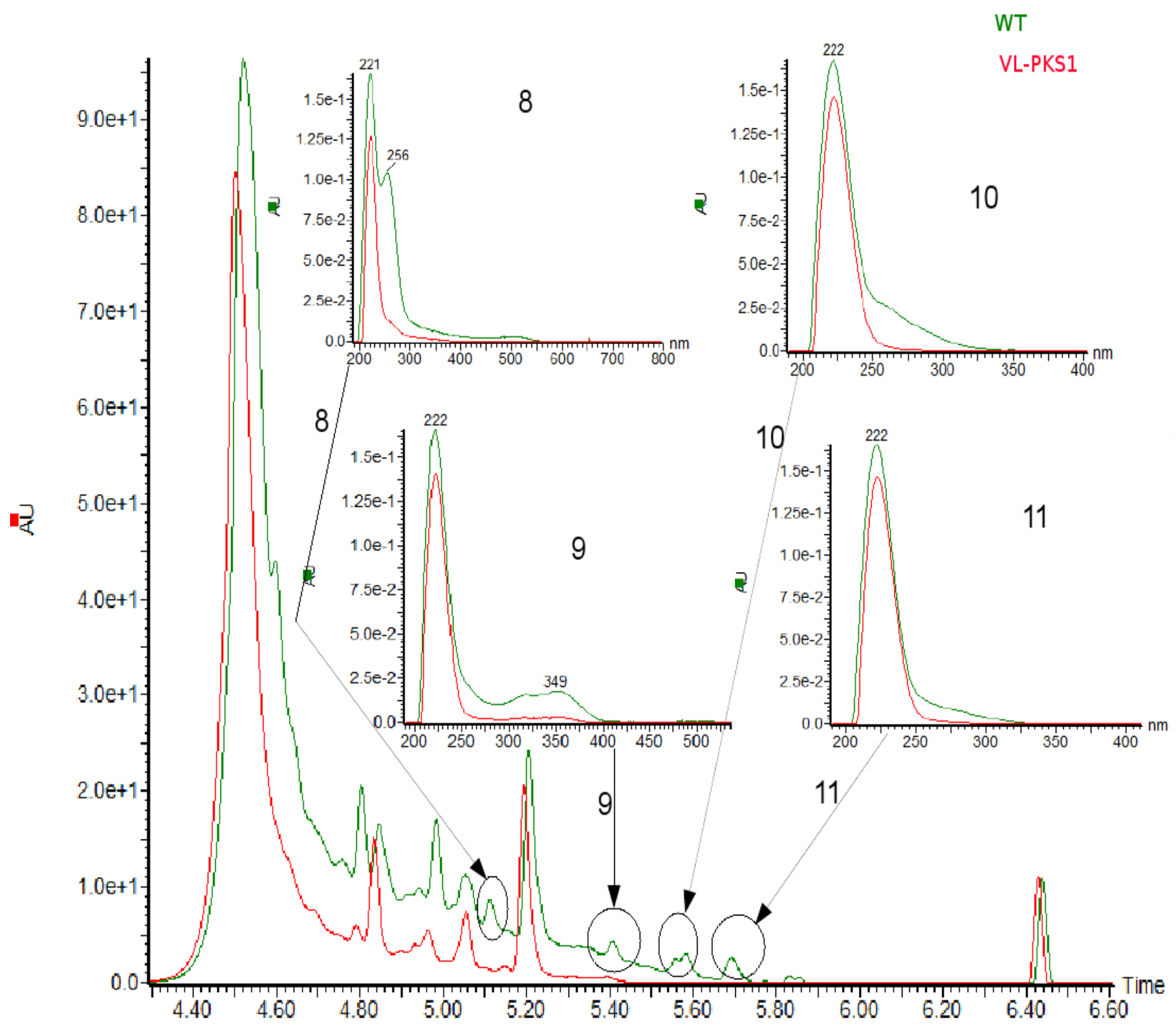

Figure 3C. UV/VIS analysis of supernatant of the $V$. longisporum wild type and VI-PKS-1 mutant

The total wavelength chromatogram (190 - $800 \mathrm{~nm}$, section 4 to $6.6 \mathrm{~min}$ ) is shown for the supernatant of $V$. longisporum wild type (green) and VL-PKS1 mutant (red). The corresponding UV/VIS absorption spectra of the signals 8-11 are shown as inlets. 
Eleven signals could be detected, which show higher signal intensity (UV/VIS absorption) in the supernatant of the wild type strain than in the strains with RNAi-silenced PKS-1 gene. For some of these signals the corresponding mass spectra detected by the time-offlight mass spectrometry (TOF-MS) were analyzed. The mass spectra, which corresponds with the UV/VIS signal 1 show the $\mathrm{m} / \mathrm{z} 263.0825[\mathrm{M}+\mathrm{H}]^{+}$in positive mode and 261.0725 $[\mathrm{M}-\mathrm{H}]^{-}$in negative mode, which was found to be enriched in the supernatant of the wild type while is not or less pronounced detectable in the extracted supernatant of the V.L PKS1-1 mutant (Fig. 4).
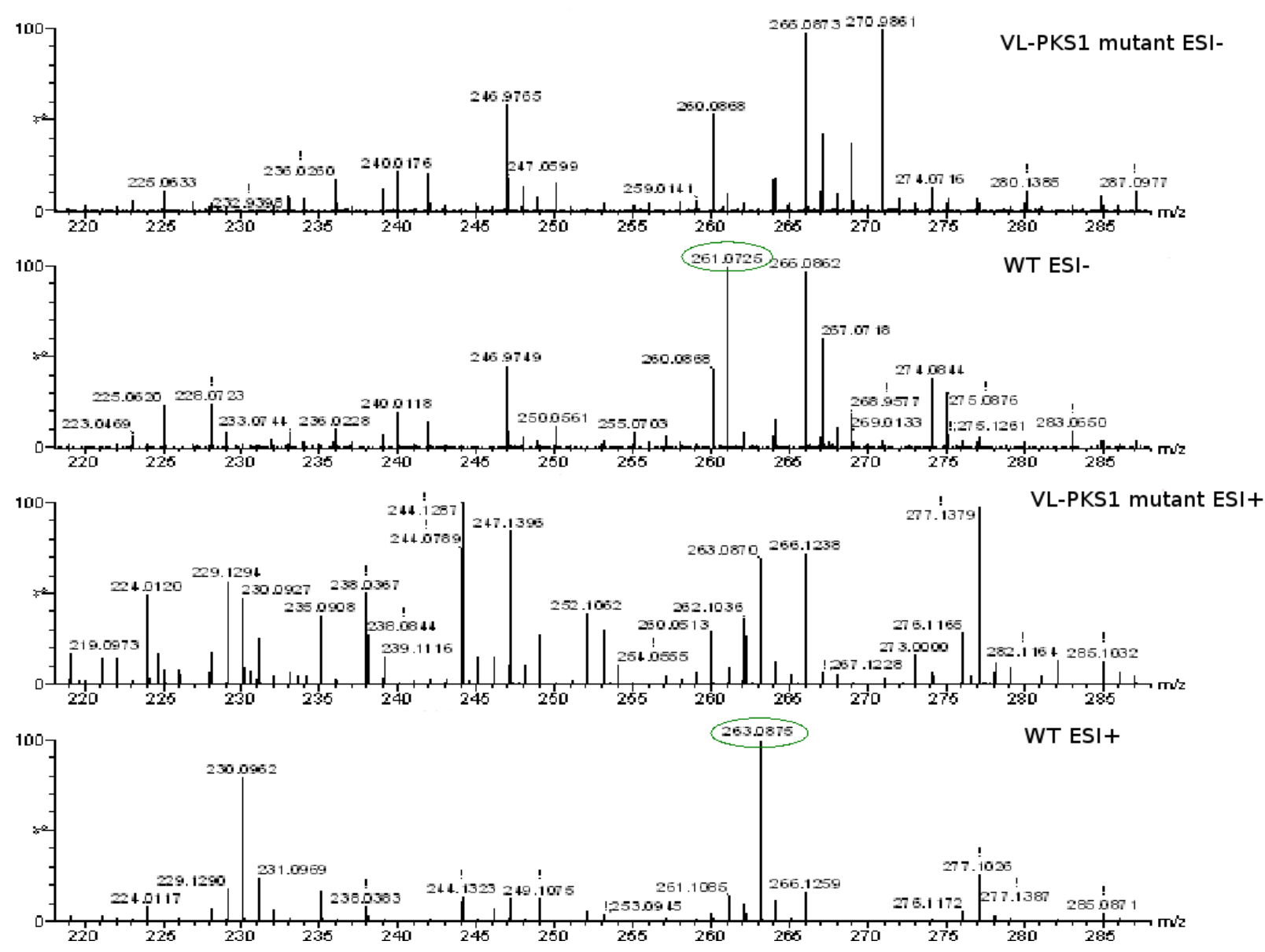

Figure 4. The mass spectra, which correspond with the signal 1 derived from the DAD detection. The spectra shows that the compound of $\mathrm{m} / \mathrm{z} 261.0725[\mathrm{M}-\mathrm{H}]^{-}$in negative and $\mathrm{m} / \mathrm{z}$ $263.0825[\mathrm{M}-\mathrm{H}]^{+}$in the positive ionization mode was found to be enriched in the wild type. 
The time-of-flight mass spectrometry (TOF-MS) of the compounds eluting with the second peak which were found in diode array detection revealed that they contained several compounds of the following mass to charge values, detected in the negative mode of ESI

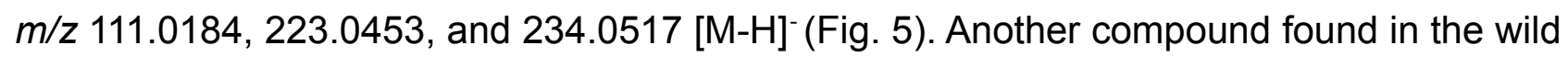
type but not present in the supernatant of the VI-PKS-1 mutant was detected in the positive mode of ESI with mass to charge $\mathrm{m} / \mathrm{z} 130.0861[\mathrm{M}+\mathrm{H}]^{+}$, this $\mathrm{m} / \mathrm{z}$ value matches several masses of known compounds including pipecolic acid, based on KEGG data base see Appendix, Table. 4
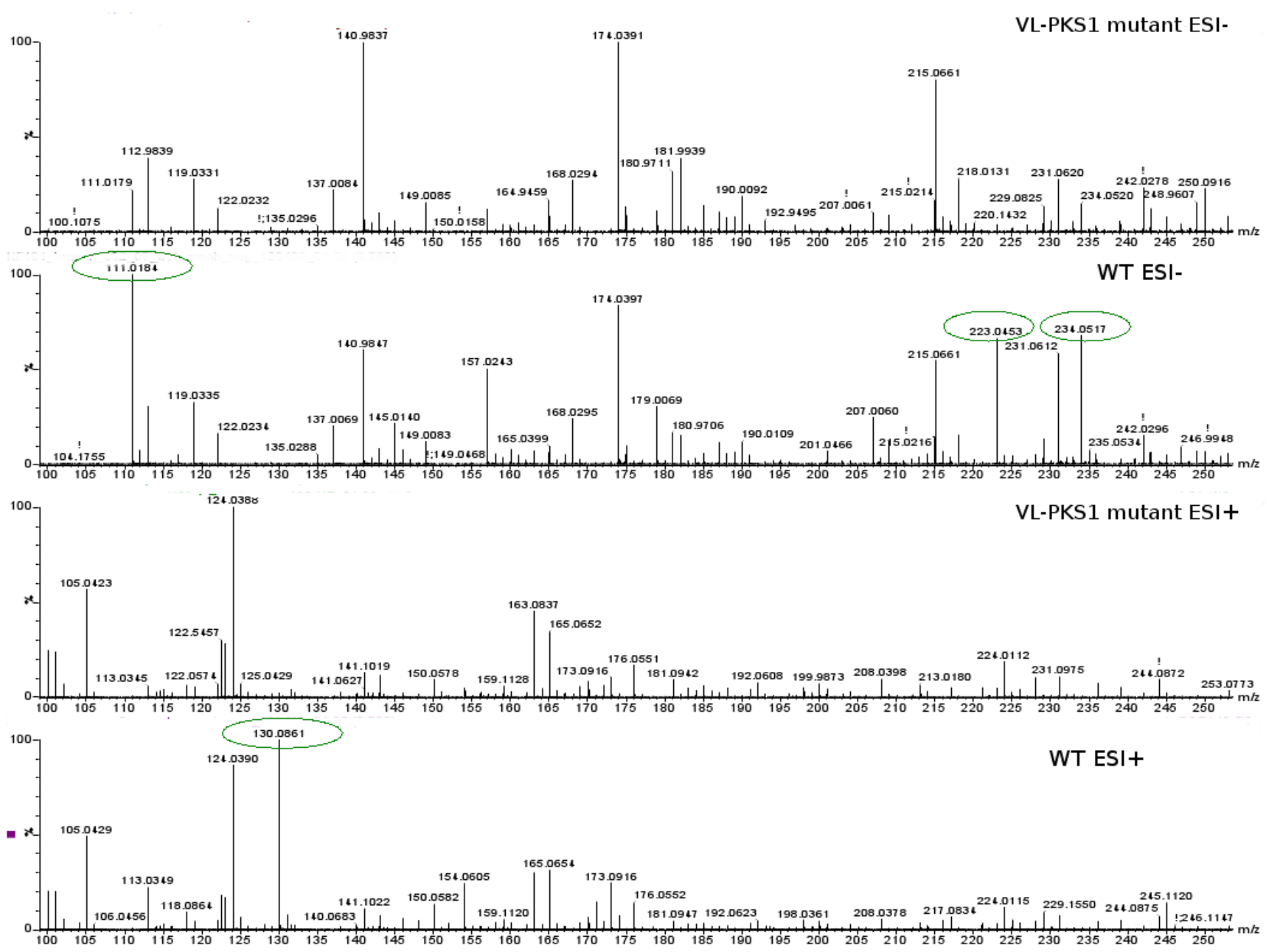

Figure 5. The mass spectra, which correspond with the signal 2 derived from the DAD detection. The compounds of mass to charge ratio of $111.0184,223.0453,234.0517[\mathrm{M}-\mathrm{H}]^{-}$in negative mode are circled in green, were present in the wild type of $V$. longisporum while they are not detectable in VL-PKS-1 mutant. Another compound of mass to charge $130.0861[\mathrm{M}+\mathrm{H}]^{+}$found in the wild type but not in the VL-PKS-1 mutant is circled in green. 
Mass spectra of the signal nr. 5 which was found by the means of diode array detector showed that it encompassed a major compound which was detected in negative mode with $\mathrm{m} / \mathrm{z}$ of $180.0657[\mathrm{M}-\mathrm{H}]^{-}$. The compound was also detectable in the positive mode with $\mathrm{m} / \mathrm{z}$ of $182.0818[\mathrm{M}+\mathrm{H}]^{+}$as shown in Fig. 6. A putative sum formula of $\mathrm{C}_{9} \mathrm{H}_{11} \mathrm{NO}_{3}$ could be concluded from the neutral monoisotopic mass of $181.074 \mathrm{Da}$., This value matches several known masses, Table 3 of Chapter 4.
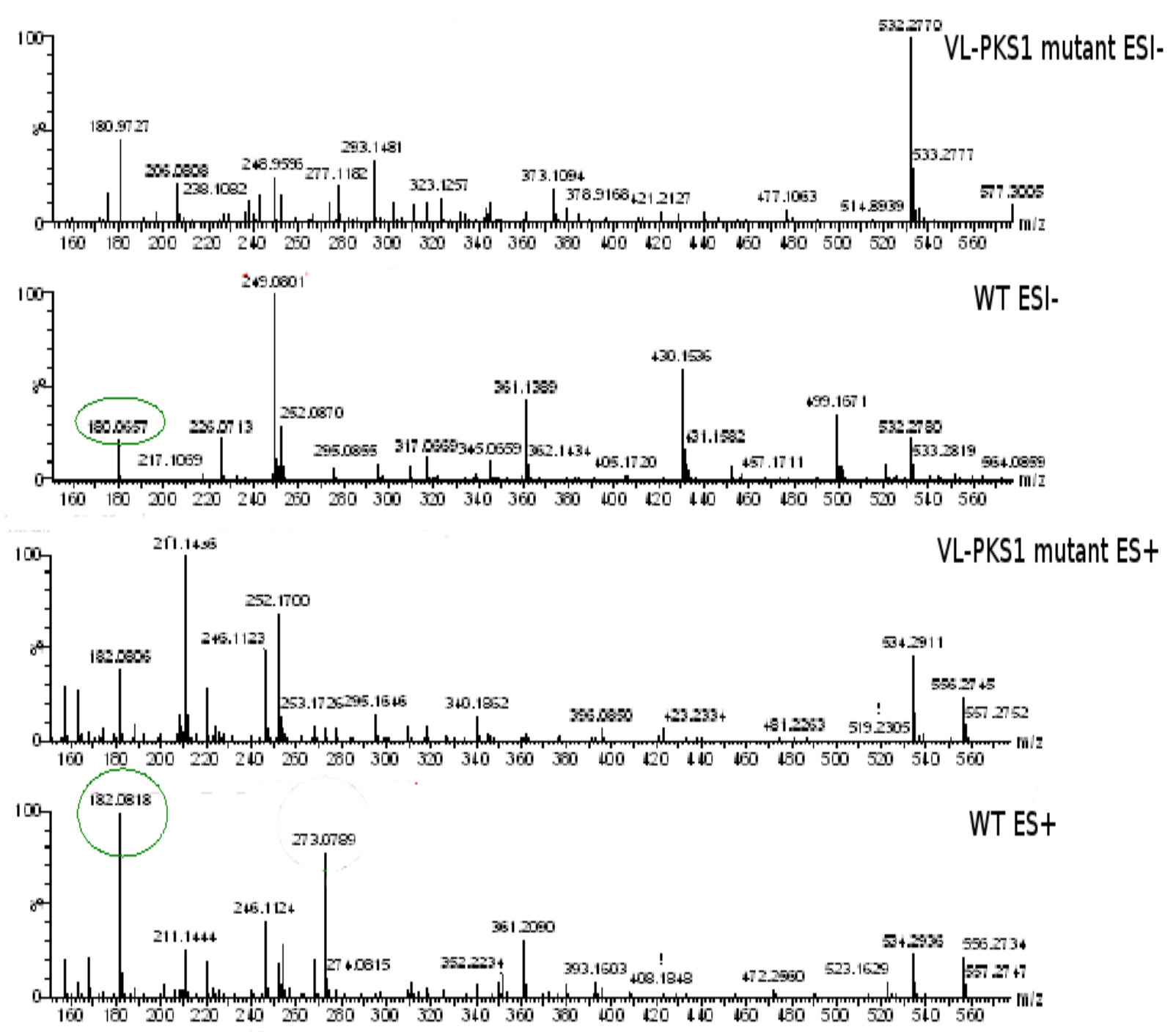

Figure 6. The extracted mass spectra of the fifth peak which was found in the DAD The compound of mass to charge ratio of $[\mathrm{M}-\mathrm{H}]^{-} 180.0657$ circled in green in negative mode, the same compound was detectable in positive mode of $\mathrm{ESI}[\mathrm{M}+\mathrm{H}]^{+} 182.0818$. 
TOF-MS of the peak 7 which was detected by diode array detection encompassed several major compounds, one of which has $\mathrm{m} / \mathrm{z}$ of $373.1872[\mathrm{M}-\mathrm{H}]^{-}$in negative mode and also could be detected in positive mode with $\mathrm{m} / \mathrm{z}$ of $375.2029[\mathrm{M}+\mathrm{H}]^{+}$. Another compound was detected only in positive mode and has the mass to charge ration of $m / z 127.07531[\mathrm{M}+\mathrm{H}]^{+}$ as shown in Fig. 7., the $\mathrm{m} / \mathrm{z}$ matches This mass to charge value matches several masses of know compounds when compared to KEGG data base, see Table.1 in Appendix of chapter 4.
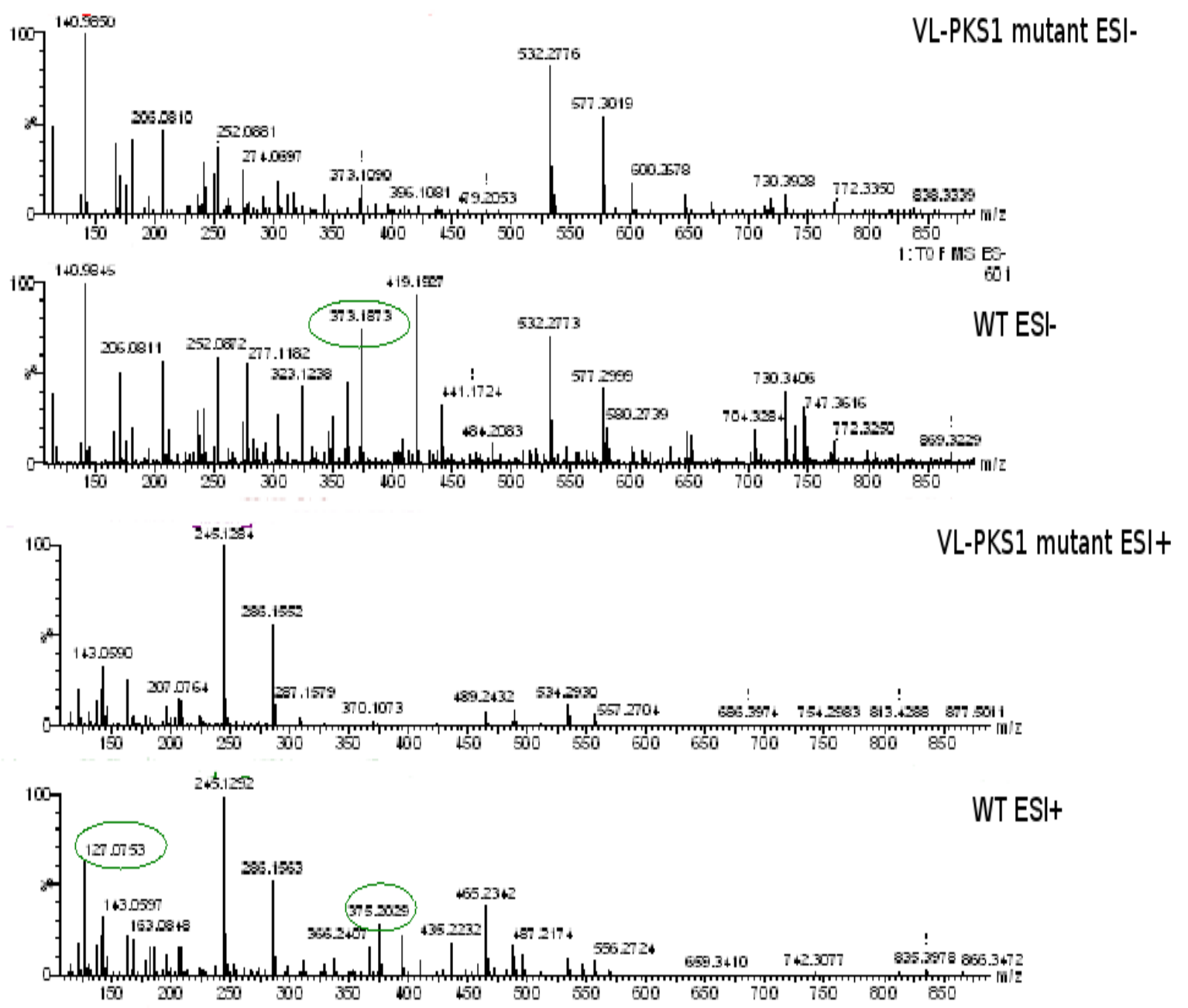

Figure 7. The extracted mass spectra of the seventh peak s which was found in DAD detection, The compound of mass to charge ratio of $\mathrm{m} / \mathrm{z} 373.1872[\mathrm{M}-\mathrm{H}]$ c circled in green in negative mode, the same compound was detectable in positive mode $\mathrm{m} / \mathrm{z}$ 375.2029.[M+H] another compound was detectable in the positive mode TOF-MS analysis of the extracted supernatant of wild type but not in the VI-PKS1 mutant. 
TOF-MS of the peak 8 which were found in diode array detector showed that it contained a major compound which had $\mathrm{m} / \mathrm{z} 178.0496[\mathrm{M}-\mathrm{H}]^{-}$in negative mode; the compound could also can be detected in positive mode of ESI with a $\mathrm{m} / \mathrm{z} 180.0652[\mathrm{M}+\mathrm{H}]^{+}$as shown in Fig. 8. This value of $\mathrm{m} / \mathrm{z} 180.0652[\mathrm{M}+\mathrm{H}]^{+}$matches several masses of know compounds when compared to KEGG data base, see table 2 in Appendix of chapter 4.
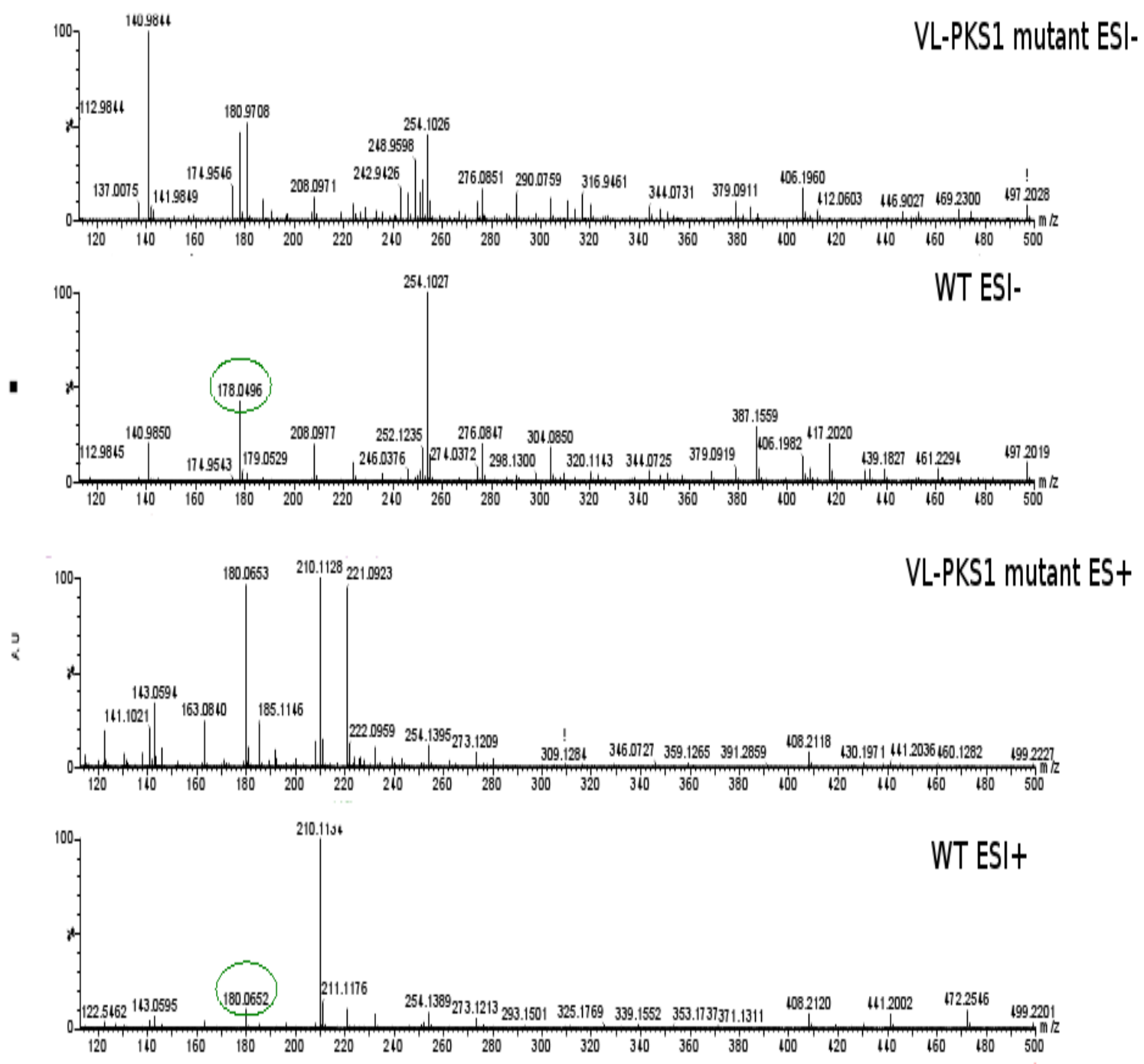

Figure 8. The extracted mass spectra of the eighth peak which was found in UV detection. The compound of mass to charge ratio of $\mathrm{m} / \mathrm{z} 178.0496[\mathrm{M}-\mathrm{H}]^{-}$circled in green in negative mode of ESI the same compound was detectable in positive mode of ESI $m / z 180.0652[\mathrm{M}+\mathrm{H}]^{+}$. 
The row TOF-MS data subjected to analysis using MarkerLynx ${ }^{\mathrm{TM}} \mathrm{XS}$ which is an Application Manager from Waters Corporation (USA) which processes complex spectrometric data and preforms data set alignment and 3-dimensional peak integration. The other software is the MarVis-Suite (Marker Visualization), which is a toolbox for analyzing intensity-based profiles of marker candidates obtained from fingerprinting/profiling. The software supports the analysis of mass spectrometry data in non-targeted metabolic fingerprinting, with functions for filtering, adduct identification and correction, data base search as well as clustering and visualization by means of onedimensional self-organizing map (1D-SOM) as described (Kaever et al., 2009; Meinicke et al., 2008).

Data analysis of MS data was carried out using MarkerLynx and MarVis-Suite. A sub set of 529 high quality marker candidates, which are enriched in the supernatant of wild type $V$. longisporum and 346 marker candidates, which are strongly deleted in the supernatant of V. longisporum PKS-1mutant are selected (Fig.9). 

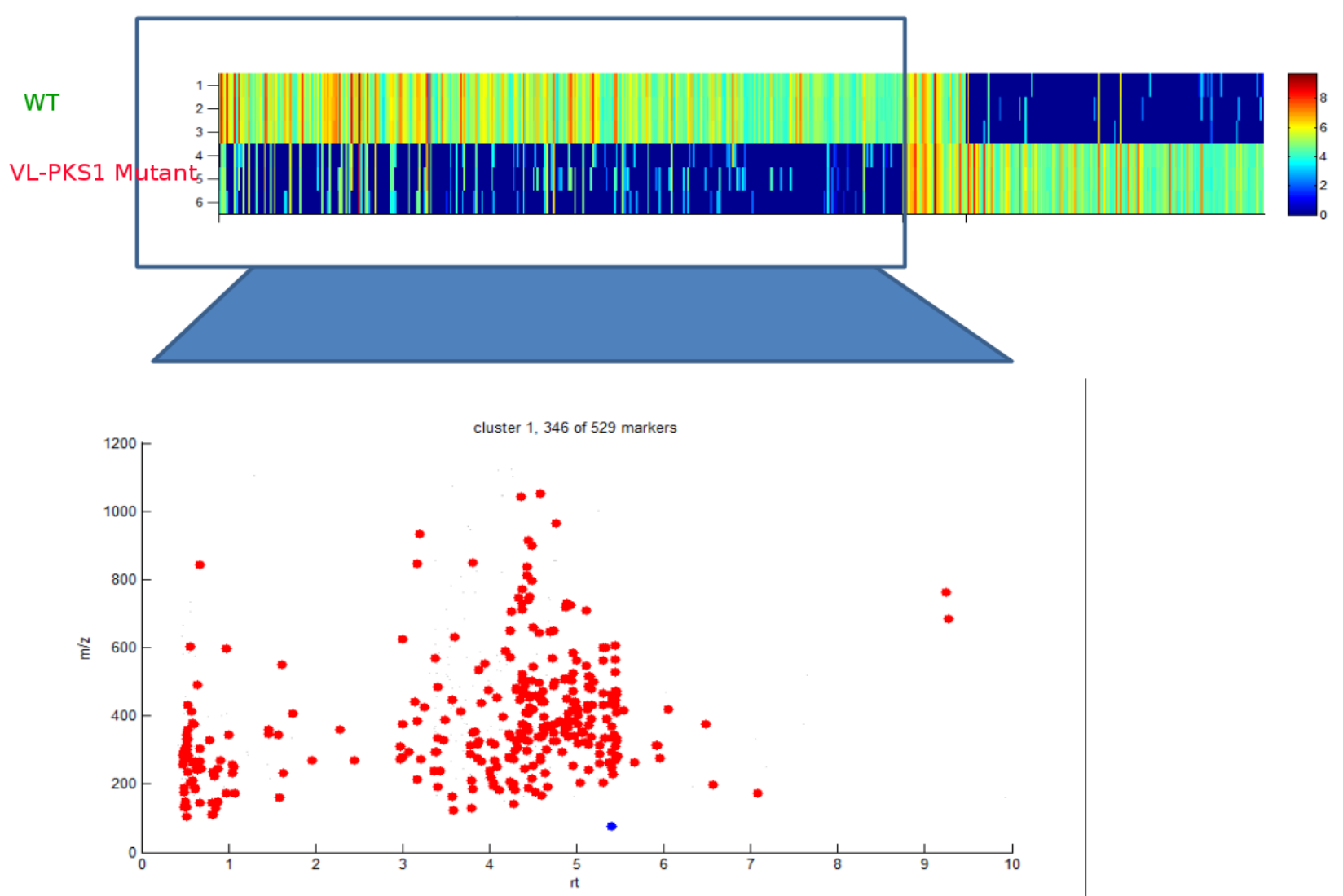

Figure 9. Metabolite fingerprinting data clustered and visualized by 1D-SOM.

The heat map of the analysis which found a cluster of 346 marker candidates were strongly deleted in the PDB supernatant of $V$. longisporum PKS-1 silenced gene, the blue color shows the metabolites which were not present or reduced, the red color indicate markers with increased intensities.

The masses found matched to the known masses in the database of KEGG: Kyoto Encyclopedia of Genes and Genomes, this resulted in several candidate compounds which have the same masses and formulas. One of these with $\mathrm{m} / \mathrm{z} 130.0861[\mathrm{M}+\mathrm{H}]^{+}$in positive mode, has the formula of $\mathrm{C}_{6} \mathrm{H}_{12} \mathrm{NO}_{2}$ which matches several compounds, one of them is pipecolic acid as shown in Table 4 in appendix of chapter 4 . 


\section{Discussion}

Polyketides are a vast class of secondary metabolites synthesized by filamentous fungi as well as by bacteria and plants, in a similar mechanism to that of fatty acid synthesis. (Hopwood and Sherman, 1990). Some of these polyketides are important mycotoxins like aflatoxin B1 (Watanabe et al., 1996). Other polyketides are responsible for pigmentation in fungi like naphthopyrone compound YWA1 in A. nidulans (Watanabe et al., 1999). VLPKS1 is a gene encodes for polyketide synthase (PKS) in the hemi-biotrophic fungus $V$. longisporum. Silencing this gene resulted in different morphological and physiological changes in transformants compared to wild type of the fungus $V$. longisporum including, faster growth rate and later pigmentation but has no effect on virulence or pathogenicity of the fungi.(Beinhoff, 2011). The aim of the study was to observe changes of the metabolic profile of $V$. longisporum compared to one of the V. longisporum silenced RNAi-gene transformant, and underline the signals and the secondary metabolites which are affected as a result of the silencing. For the study we used HPLC-MS initially to underline the differences then we used light absorption detector combined with TOF mass spectrometry.

The masses of the signals which we found that they changed between the wild type and the VL-PKS1 mutant in the initial HPLC-MS analysis were different from the masses that were found in HPLC-TOF-MS analysis, The reason is that we used different methods of extraction. While in HPLC-MS analysis, ethyl acetate was used, in the UV/VIS combined with TOF-MS, a two phase extraction of methanol/water/methyl-tert-butylether were used, which is suitable for polar as well as non polar compounds. However the compound(s) we presume to be responsible for the discoloration of the PDB medium should be polar.

In the second part of the analysis we selected 11 peaks from UV/VIS analysis which were enriched or present in the wild type and not in the VL-PKS-1 silenced type of the fungi. Then we examined the spectra of corresponding masses and matched the masses with known compounds in the database KEGG: Kyoto Encyclopedia of Genes and Genomes. The comparison resulted in several candidate compounds which have the same masses and sum formulas, see Appendix of chapter 4. One of these candidate compounds is pipecolic acid, a small molecule known to be produced in plants, animals, and microbes (Broquist, 1991). Relationship between the PKS gene and the lysine requires further investigation. 
None of the peaks identified in HPLC-Diode array detection absorbed light in the red part of the spectrum. We hypothesized that the metabolite(s) responsible for the discoloration of PDB medium was/were not detected by HPLC-DAD because it was not extracted into the HPLC injected samples.

In addition to the analysis of HPLC-DAD spectra we compared the raw MS data using the software MarkerLynx and MarVis. In this system, metabolic signals with representation significant differences between the two strains are called marker candidates. A total of 346 marker candidates were strongly reduced in the mutant type of VL-PKS-1 silenced gene. This is obviously much more than can be explained biochemically as a consequence of silencing a single PKS gene. Part of these signals may be adducts and/or in-source fragmentation products of metabolites genuinely reduced in the extracts of the mutant strain. The identification of the products would require further analysis. Unfortunately gene silencing by RNAi does not abolish transcription of the target genes completely so that on protein level a residual enzymatic activity could be present. For that reason also basal amounts of the corresponding enzymatic derived metabolites can be still present in the mutant.

The software analysis also found that there are enhanced compounds in the mutant type of $V$. longisporum-PKS1, this can be explained that common precursors are available for other alternative pathways, resulting in enhanced synthesis of other metabolites. 


\section{Outlook}

Metabolic profiling of the $V$. longisporum wild type in comparison with VL-PKS1 mutant grown in different medium should be investigated to confirm if the same masses are persist in different medium which showed no discoloration like SXM medium. Then we can exclude the compounds which are not involved in this observation. Tandem mass spectrometry (MS/MS) analysis of the candidate compounds will help to improve the identification of the these candidate compounds. Purification of these candidate compounds will confirm the structure elucidation.

\section{Acknowledgments}

We thank Prof. Dr. Ivo Feussner (Department of Plant Biochemistry, Albrecht-von-Haller Institute for Plant Sciences, Georg-August-University, Göttingen) for conducting part of this study in his laboratory, and Pia Meyer for extracting the samples for UVIVIS and TOF-MS analysis. Dr. Kirstin Feussner (Department of Plant Biochemistry, Albrecht-von-Haller Institute for Plant Sciences, Georg-August University, Göttingen) who carried out the UPLC-DAD, TOF-MS analysis.

\section{References}

Aliferis, K.A., Cubeta, M.A., Jabaji, S., 2013. Chemotaxonomy of fungi in the Rhizoctonia solani species complex performing GC/MS metabolite profiling. Metabolomics 9, 159-169.

Beck, J., Ripka, S., Siegner, A., Schiltz, E., Schweizer, E., 1990. The multifunctional 6methylsalicylic acid synthase gene of Penicillium patulum. Eur. J. Biochem. 192, 487-498.

Beinhoff, M., 2011. Molecular and functional characterization of potential pathogenicity related genes from Verticillium longisporum. Georg-August-Universität Göttingen, Göttingen, Germany. 
Bertrand, S., Schumpp, O., Bohni, N., Bujard, A., Azzollini, A., Monod, M., Gindro, K., Wolfender, J.-L., 2013. Detection of metabolite induction in fungal co-cultures on solid media by high-throughput differential ultra-high pressure liquid chromatography-time-offlight mass spectrometry fingerprinting. J.Chromatography A. 1292, 219-228.

Bingle, L.E., Simpson, T.J., Lazarus, C.M.,1999. Ketosynthase domain probes identify two subclasses of fungal polyketide synthase genes. Fungal Genet. Biol. 26, 209-223.

Broquist, H.P., 1991. Lysine-Pipecolic Acid Metabolic Relationships in Microbes and Mammals. Annu. Rev. Nutr. 11, 435-448.

Dunn, W.B., Hankemeier, T., 2013. Mass spectrometry and metabolomics: past, present and future. Metabolomics 9, 1-3.

Eynck, C., Koopmann, B., Grunewaldt-Stoecker, G., Karlovsky, P., Tiedemann, A. von, 2007. Differential interactions of Verticillium longisporum and V. dahliae with Brassica napus detected with molecular and histological techniques. Eur. J. Plant Pathol. 118, 259274.

Floerl, S., Majcherczyk, A., Possienke, M., Feussner, K., Tappe, H., Gatz, C., Feussner, I., Kües, U., Polle, A., 2012. Verticillium longisporum Infection Affects the Leaf Apoplastic Proteome, Metabolome, and Cell Wall Properties in Arabidopsis thaliana. Plos One 7 , e31435.

Hopwood, D.A., Sherman, D.H., 1990. Molecular Genetics of Polyketides and its Comparison to Fatty Acid Biosynthesis. Annu. Rev. Genet. 24, 37-62.

Kaever, A., Lingner, T., Feussner, K., Göbel, C., Feussner, I., Meinicke, P., 2009. MarVis: a tool for clustering and visualization of metabolic biomarkers. BMC Bioinformatics $10,92$.

Karapapa, V.K., Bainbridge, B.W., Heale, J.B., 1997. Morphological and molecular characterization of Verticillium longisporum comb, nov., pathogenic to oilseed rape. Mycol. Res. 101, 1281-1294.

Keller, N.P., Turner, G., Bennett, J.W., 2005. Fungal secondary metabolism — from biochemistry to genomics. Nat. Rev. Microbiol. 3, 937-947. 
Meinicke, P., Lingner, T., Kaever, A., Feussner, K., Göbel, C., Feussner, I., Karlovsky, P., Morgenstern, B., 2008. Metabolite-based clustering and visualization of mass spectrometry data using one-dimensional self-organizing maps. Algorithms Mol. Biol. 3, 9 .

Smedsgaard, J., Frisvad, J.C., 1996. Using direct electrospray mass spectrometry in taxonomy and secondary metabolite profiling of crude fungal extracts. J. Microbiol. Methods 25, 5-17.

Stadler, M., Keller, N.P., 2008. Paradigm shifts in fungal secondary metabolite research. Mycol. Res. 112, 127-130.

Stergiopoulos, I., Collemare, J., Mehrabi, R., De Wit, P.J.G.M., 2013. Phytotoxic secondary metabolites and peptides produced by plant pathogenic Dothideomycete fungi. Fems Microbiol. Rev. 37, 67-93.

Tsuge, T., Harimoto, Y., Akimitsu, K., Ohtani, K., Kodama, M., Akagi, Y., Egusa, M., Yamamoto, M., Otani, H., 2013. Host-selective toxins produced by the plant pathogenic fungus Alternaria alternata. Fems Microbiol. Rev. 37, 44-66.

Watanabe, A., Fujii, I., Sankawa, U., Mayorga, M.E., Timberlake, W.E., Ebizuka, Y., 1999. Re-identification of Aspergillus nidulans wA gene to code for a polyketide synthase of naphthopyrone. Tetrahedron Lett. 40, 91-94.

Watanabe, C.M.H., Wilson, D., Linz, J.E., Townsend, C.A., 1996. Demonstration of the catalytic roles and evidence for the physical association of type I fatty acid synthases and a polyketide synthase in the biosynthesis of aflatoxin B1. Chem. Biol. 3, 463-469.

Zeise, K., Tiedemann, A. von, 2001. Morphological and Physiological Differentiation among Vegetative Compatibility Groups of Verticillium dahliae in Relation to $V$. longisporum. J. Phytopathol. 149, 469-475. 


\section{Appendix of chapter 4}

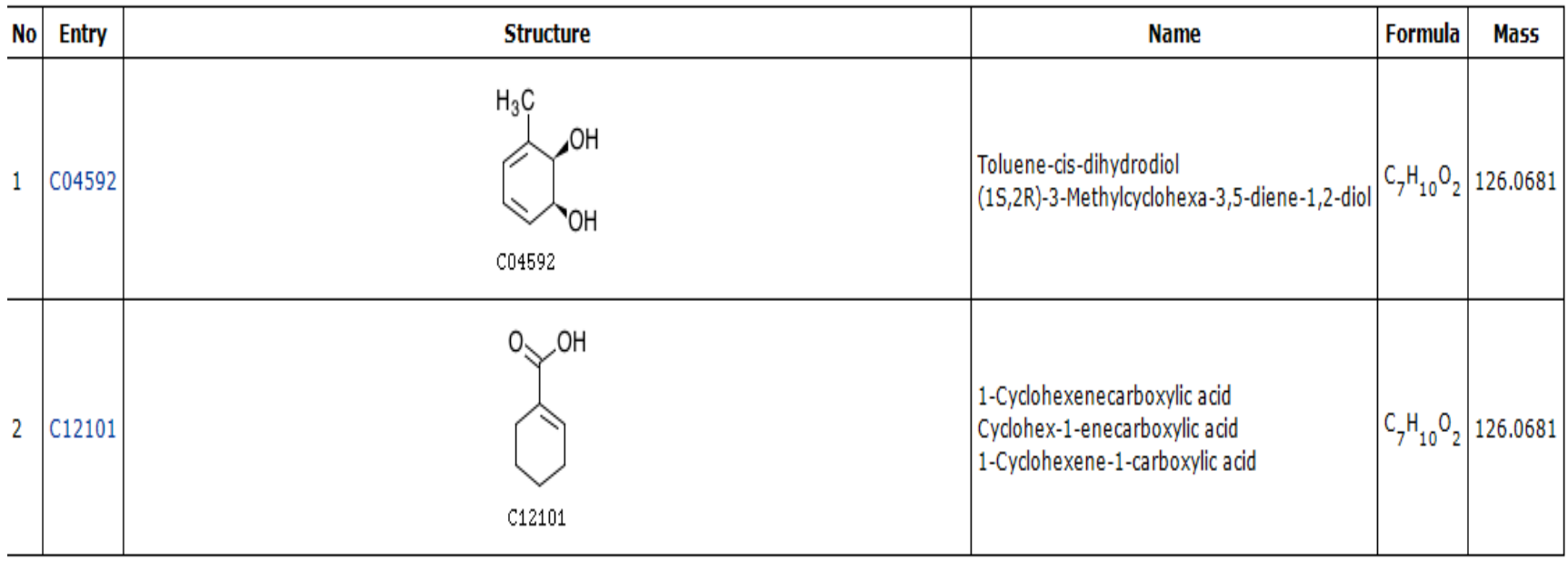

Table.1. Masses of known compounds matched the identified compound of mass to charge $m / z$ 127.07531[M+H] $]^{+}$

Source of data base: KEGG: Kyoto Encyclopedia of Genes and Genomes, (http://www.genome.jp/kegg/ligand.html)

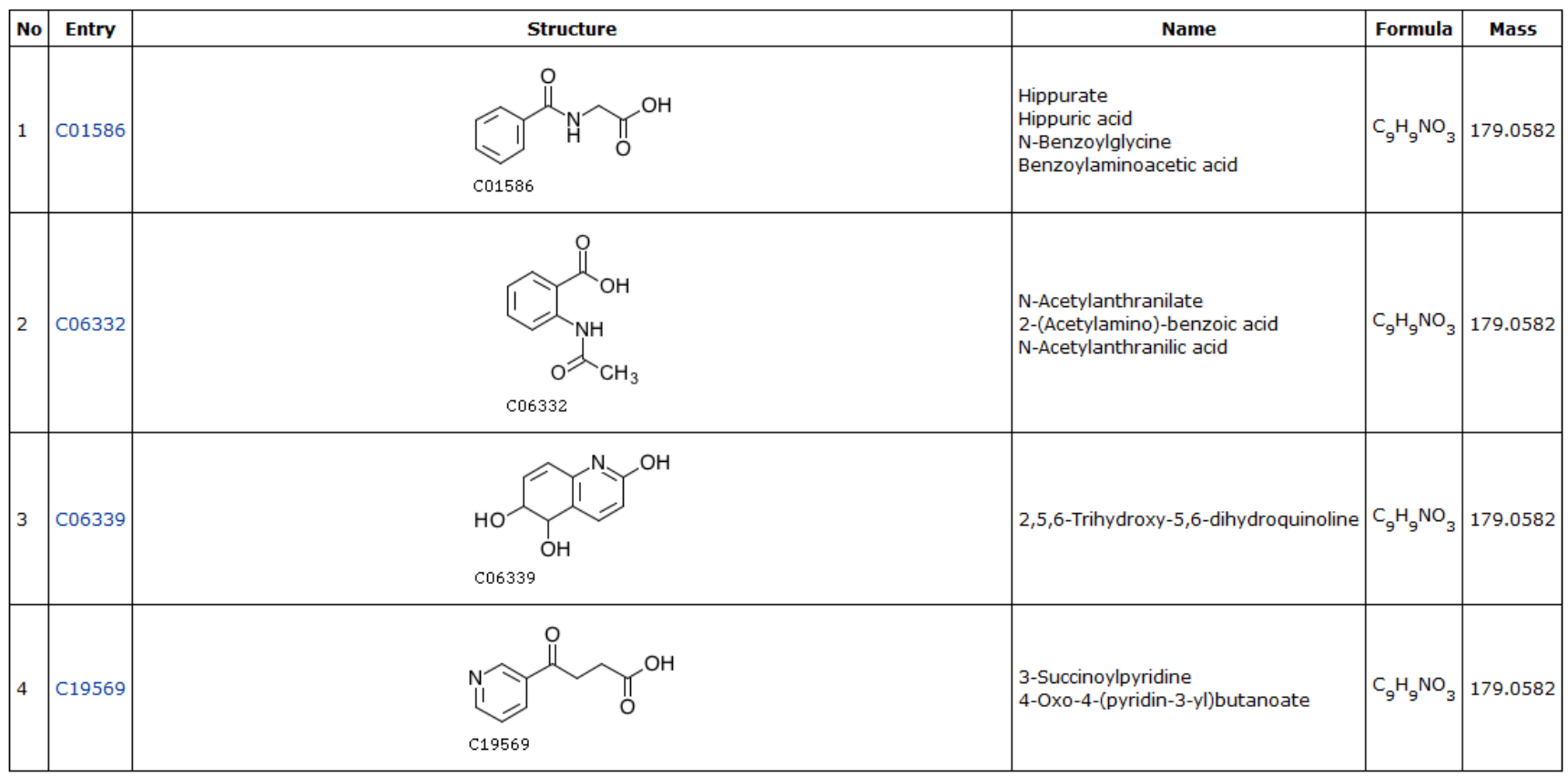

Table.2. Masses of known compounds matched the identified compound of mass to charge $m / z 180.0652[\mathrm{M}+\mathrm{H}]+$

Source of data base: KEGG: Kyoto Encyclopedia of Genes and Genomes,

(http://www.genome.jp/kegg/ligand.html) 


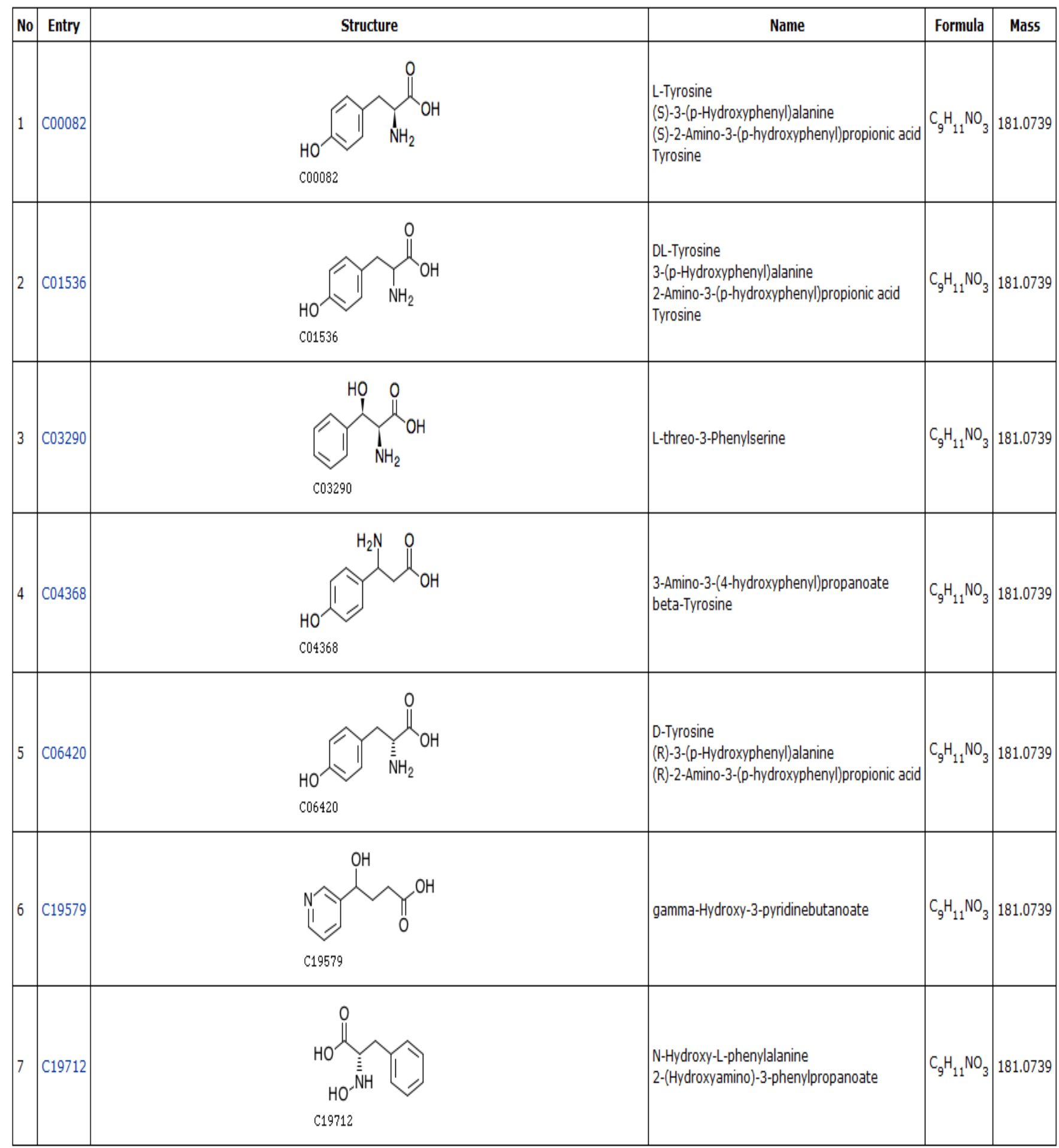

Table.3. Masses of known compounds matched the identified compound of mass to charge $m / z$ 182.0818[M+H] $]^{+}$Source of data base: KEGG: Kyoto Encyclopedia of Genes and Genomes, (http://www.genome.jp/kegg/ligand.html 


\begin{tabular}{|c|c|c|c|c|}
\hline No & Entry & Structure & Name & Formula \\
\hline 1 & C00408 & C00408 & $\begin{array}{l}\text { L-Pipecolate } \\
\text { Pipecolinic acid } \\
\text { Pipecolic acid } \\
\text { 2-Piperidinecarboxylic acid } \\
\text { (S)-Piperidine-2-carboxylic acid }\end{array}$ & $\mathrm{C}_{6} \mathrm{H}_{11} \mathrm{NO}_{2}$ \\
\hline 2 & C03969 & C03969 & $\begin{array}{l}\text { 1-Aminocyclopentanecarboxylate } \\
\text { 1-Aminocyclopentanecarboxylic acid } \\
\text { Cycloleucine }\end{array}$ & $\mathrm{C}_{6} \mathrm{H}_{11} \mathrm{NO}_{2}$ \\
\hline 3 & C05936 & C05936 & $\begin{array}{l}\text { N4-Acetylaminobutanal } \\
\text { 4-Acetamidobutanal }\end{array}$ & $\mathrm{C}_{6} \mathrm{H}_{11} \mathrm{NO}_{2}$ \\
\hline 4 & C07500 & $\begin{array}{l}\mathrm{H}_{2} \mathrm{C}^{->} \\
\mathrm{C} 07500\end{array}$ & Vigabatrin & $\mathrm{C}_{6} \mathrm{H}_{11} \mathrm{NO}_{2}$ \\
\hline 5 & C12829 & $\begin{array}{l}\mathrm{H}_{3} \mathrm{C} \\
\mathrm{C} 12829\end{array}$ & \begin{tabular}{|l} 
Bromisovalum \\
Bromovalerylurea
\end{tabular} & $\mathrm{C}_{6} \mathrm{H}_{11} \mathrm{BrN}_{2} \mathrm{O}_{2}$ \\
\hline 6 & C13696 & C13696 & trans-3-Aminocyclopentane-1-carboxylic acid & $\mathrm{C}_{6} \mathrm{H}_{11} \mathrm{NO}_{2}$ \\
\hline
\end{tabular}

Table.4. Masses of known compounds matched the identified compound of mass to charge $m / z$ 130.0861 [M+H] $]^{+}$Source of data base: KEGG: Kyoto Encyclopedia of Genes and Genomes, (http://www.genome.jp/kegg/ligand.html). 


\section{Chapter 5: General discussion}

In this study we established a method for isolation and purification of secondary metabolites which are specific for $V$. longisporum infection. These secondary metabolites occur in the plant-pathogen system $V$. longisporum/B. napus specifically in the xylem sap of $B$. napus, where the biochemical interactions between the pathogen and the host plant take place. We developed an assay to understand the effect of these small molecules in xylem sap against other microorganisms. Moreover, we observed changes in the metabolic profile of $V$. longisporum and the affected secondary metabolites when VI-PKS-1 gene is silenced, which was previously identified as a gene encoding for polyketide synthase.

B. napus, which is the host plant involved in this study is a major crop cultivated worldwide for nutritional vegetable oil production, in addition to other industrial uses. Soil-borne diseases pose a serious problem in oilseed rape cultivation. The pathogen we discussed this study was $V$. longisporum, it is one of the most important soil-borne pathogens which cause Verticillium disease on B. napus (Karapapa et al., 1997). It has become a serious threat to oilseed cultivation in northern Europe (Johansson et al., 2006). It is also considered as one of the potential pathogens in northern Germany which may benefit from increasing temperature due to global warming.(Siebold and Tiedemann, 2012) and it can lead to significant losses in the yield (Dunker et al., 2008). and it is the most recent plant pathogenic fungi from Verticillium species, evolved through hybridization from other Verticillium species (Inderbitzin et al., 2011), which are notorious vascular pathogenes causing vascular diseases in a wide range of crops in different parts of the world (Bhat and Subbarao, 1999; Klosterman et al., 2009; Pegg and Brady, 2002).

Improvement of resistance towards diseases is one of the best strategies to overcome economic losses caused by Verticillium diseases in oilseed rape, especially since there is no effective chemical control available for these diseases. To improve resistance toward diseases, it is necessary to understand the metabolic pathways involved in the plant defense mechanism, which play a key role in both chemically induced resistance and genotype related resistance in B. napus (Kamble et al., 2012; Obermeier et al., 2013). Despite of the fact that secondary metabolites do not participate directly in the growth and 
development of living organisms, they seem to be vital for their survival. Both plants and microorganisms produce a broad spectrum of secondary metabolites which play a key role in the ecological interactions with their environment. In plant/pathogen system, plants produce these low molecular weight compounds as response the microbial attack (Kutchan, 2001; Springob and Kutchan, 2009).

In V. longisporum/B. napus, these secondary metabolites posses several functions. Some of these functions are known but others are still undiscovered. Phytohormones act as signals which trigger systemic resistance toward the invading $V$. longisporum as part of the plant defense mechanism. Salicylic acid (SA) and salicylic acid glucoside (SAG) levels are reported to be increased after $V$. longisporum infection in B. napus (Ratzinger et al., 2009). However, the increased levels of SA and SAG are not related to disease resistance in V. Iongisporum /B. napus system (Kamble et al., 2012). Other phytohormones like ethylene seem to trigger synthesis of defensive enzymes in B. napus (Floerl et al., 2008). Furthermore, other known roles of secondary metabolites in V. longisporum/B.napus like phenolics, lignen, lignin like compounds accumulate after $V$. longisporum infection in the vascular system in a bid to stop the pathogen colonization of xylem vessels (Eynck et al., 2009). The phenylpropanoid pathway and some of them play a key role in resistance in $V$. longisporum/ B. napus and V. Iongisporum resistance (Kamble et al., 2012; Obermeier et al., 2013).

While the aforementioned roles of phytohormones and phenylpropanoids seem to be well investigated in previous studies of $V$. longisporum/B. napus, the role of phytoalexins, another important class of secondary metabolites involved in plant defense; is still unclear. This study aimed to disclose the role of phytoalexins in $B$. napus defense against $V$. longisporum.

It is known that $B$. napus produces phytoalexins in a response to pathogens. The soil borne pathogenic fungus Plasmodiophora brassicae elicits the production of blends of phytoalexins in the root of $B$. napus, while these phytoalexins were not observed in the leaves. Cyclobrassinin is one of the most abundant among these phytoalexins (Pedras et al., 2008). We wanted to chemically investigate these phytoalexins in V. longisporum/ $B$. napus system. Xylem sap is the niche where chemical interactions between $V$. longisporum and $B$. napus take place, due to the biotrophic style of $V$. longisporurm which 
colonizes the xylem vessels of $B$ napus (Eynck et al., 2007). Therefore, the plant is expected to secret phytoalexins into the xylem sap targeting the fungi. In a previous study non-targeting metabolic profiling of xylem sap of $V$. longisporum infected and non infected $B$. napus revealed eight signals which are specific for $V$. longisporum infection. These masses did not match with any of the known $B$. napus phytoalexins, but they possess common building blocks based on indole derivatives (Ratzinger, 2008). Therefore, this study complements the previous study and chemically characterizes these compounds. Isolation and purification of these secondary metabolites is the only way to achieve the chemical characterization of these compounds. It is known that purification of cruciferous phytoalexins is not straightforward task, because of the small amounts of these compounds present and also due to the complicated plant matrix resulting from the injured plants. Furthermore, the stability of the purified compounds is a major problem in this process, (Pedras and Yaya, 2010; Pedras et al., 2011, 2006) However, we conducted the isolation from xylem sap which is less complex than the plant matrix. In order to start the purification, large amounts of xylem sap was required, we collected xylem sap of a large number of $B$. napus rapid cycling plants, which have a shorter life cycle than regular rape plants and are easier to handle. The amount of xylem sap collected from each plant was very little, the work was laborious and time consuming, therefore improvement of the method of xylem sap extraction for an increased yield in still required. We established a purification method of secondary metabolites from $B$. napus which can be extended to the xylem from other plants.

The method was based on preparing xylem sap for purification by removing large molecules using filter membrane, defatting and freeze drying which enabled us to concentrate larger amounts of xylem sap. The petrifaction was conducted by upgrading the analytical system which was used for detecting these compounds in a preparative HPLC system, followed by semi analytical system which guarantee removing undesired compounds obtained from the first step, see Fig 7 of Chapter 2. The purified compound which had the exact mass of $612.1197 \mathrm{Da}$, was detectable by means of HPLC-MS in both positive and negative modes of ESI (Ratzinger, 2008). We noticed that it undergoes fragmentation in the first step in favor of an increasing amount of the compound $\mathrm{m} / \mathrm{z}$ $527.1291[\mathrm{M}+\mathrm{H}]^{+}$, in a previous study conducted by (Ratzinger, 2008) both compounds are detectable in positive and negative modes of ESI. When the two compounds are subjected 
to mass spectrometric fragmentation by means of tandem mass spectrometry the two compounds show a neutral loss of of 204 amu, which is believed to be tryptophan, and they lead to the same fragments of $\mathrm{m} / \mathrm{z} 162$ which are indole-3-carboxylate moieties and m/z 118 in $\mathrm{MS}^{4}$ (Ratzinger, 2008), see Fig 11 in Chapter 2.

Therefore we believe that the mass of $m / z 527.1291[\mathrm{M}+\mathrm{H}]^{+}$is a fragment of the purified compound of the exact mass $612.1197 \mathrm{Da}$. The presumed compound shows homology to the structure of cyclobrassinin, (see Fig.4 of chapter 1), which was previously described as the most abundant phytoalexin in $B$. napus in response to $P$. brassicae, (Pedras et al., 2008) Therefore we believe that it is a B.napus phytoalexin. The purified amount of the compound was not sufficient for standard NMR analysis. Higher resolution NMR analysis is still required. We are in the process of doing so, this will confirm the chemical characterization of the purified compound.

This purified compound could be a novel phytoalexin or a detoxified form of another phytoalexin as it is known that other phytopathogenic fungi detoxify cruciferous phytolalexins (Pedras and Ahiahonu, 2005; Pedras and Hossain, 2006; Pedras et al., 2011).

We developed an assay to examine the effect of $V$. longisporum infection against other microorganisms, the bacterium $A$. tumefaciens and the yeast $S$. cerevisiae when they grow in xylem sap of $B$. napus. We wanted to answer the question, if there any inhibition effect in xylem sap due to $V$. longisporum infection, and if yes, if this effects are due to the small molecules present in xylem sap of $B$. napus. Despite of the changes observed in xylem after V. longispurm infection (Ratzinger et al., 2009), the infection had no effect on the nutrient in the xylem, which was still a sufficient medium for the growth of the two microorganisms studied. There was only an inhibition effect against $A$. tumefaciens after 24 hours of inoculation, see Fig. 2 and Fig. 3 of chapter 3 . This effect was eliminated after removal of macromolecules larger than 3.000 Da., see Fig. 4 of chapter 3. Therefore, there is an inhibition effect on the bacterium $A$. tumefaciens and this affect is due to the large molecules rather than due to small molecular weight secondary metabolites present in the xylem sap of infected $B$. napus. It is obvious that the bacterium was inhibited by the proteins present in xylem sap of $B$. napus. The xylem sap of $B$. napus is known to contain a number of proteins (Kehr et al., 2005). Furthermore, V. longisporum infection triggers the 
upregulation of several defensive proteins including the endochitinase, peroxidase, PR4, and $\beta-1,3$-glucanase in the xylem sap of $B$. napus. These macromolecules are produced by the plant to inhibit $V$. longisporum (Floerl et al., 2008), some of these proteins are known to be elicited by bacteria in Brassica like PR4 (Ryang et al., 2002).

However, our study does not offer a reliable assay to examine the antimicrobial effect of small molecules or phytoalexins present in the xylem sap of $B$. napus due to many factors. One of these factors is the stability of the phytoalexins under the conditions of the experiment, which is a crucial factor in these de novo plant produced compounds (Pedras and Yaya, 2010). Probably these compounds no longer possess antimicrobial activity due to fragmentation under the high temperature, and the light conditions during the assay. Furthermore, these compounds are subjected to oxidation under the conditions of the experiment. Another factor is the specificity of this phytoalexins or the blends of these phytoalexins against the targeted microbe. One more factor, which could make these small molecules inactive is the detoxification of these compounds by the pathogen, especially if we know that several phytopathogens are able to detoxify or metabolize these cruciferous phytoalexins (Pedras and Ahiahonu, 2002, 2005; Pedras and Hossain, 2006; Pedras et al., 2009). Examining small compounds in the xylem sap of infected $B$. napus for antimicrobial activities, is still not possible without purification and chemical characterization of these compounds.

So far we discussed secondary metabolites in $V$. longisporum/B. napus from the plant side: However, from fungal side, it is expected that $V$. longisporum secrets secondary metabolites to facilitate its invasion of the host plant. Generally, biotrophic pathogens, which are obliged to live in the host plant cell do not produce phytotoxins, while necrotrophic pathogens secrete lethal toxins to kill the cells of their host plants (de Wit, 2013). The shift in the life cycle of $V$. longisporum from the biotrophic to the necrotrophic phase, suggests that the fungus changes its metabolic profile through the disease phases. 
There is little known about secondary metabolites from Verticillium species. A previous study reported that endophytes from the genus Verticillium produced secondary metabolites, which possessed anti-fungal activity (You et al., 2009). There are no reports about secondary metabolites isolated from $V$. longisporum, therefore, the role of $V$. longisporum secondary metabolites remains unclear. In this study, we initiate the first step for a better understanding of the ecological role of fungal secondary metabolites from the pathogen $V$. longisporum. We observed changes in the metabolic profile of the wild type of the fungus in comparison to mutant type of $V$. longisporum resulting from silencing the Gene VI-PKS-1, which was identified as an encoding gene of polyketide synthase (PKSs). Polyketides are the largest family of fungal secondary metabolites and many of them are proven to play an ecological role in other fungi, such as mycotoxins, like aflatoxins, and zearalenone, or in pigmentation(Kim et al., 2005; Watanabe et al., 1999, 1996). We observed changes in the discoloration of the PDB medium in the case of the wild type $V$. longisporum, see Fig 1 in Chapter 4. This confirmed one of the morphological and physiological changes which were found in a previous study (Beinhoff, 2011), due to VIPKS-1 silencing. UV/VIS analysis of both wild type and VI-PKS-1 mutants showed that several peaks were reduced due to the silencing Non of these metabolites seem to be involved in the reddish discoloration of the medium observed in wild type and not in the mutant. See Fig 3a, b, $c$ in chapter 4 . The analysis revealed that there are a number of signals reduced in the metabolic profile of $V$. longisporum on PDB medium, due to the silencing. None of the masses we selected matched masses of known toxins.

It seems that these secondary metabolites, which are affected by VI-PKS-1 silencing in $V$. longisporum/B.napus system do not play a role as virulence or pathogenicity factors. Nevertheless, it can be speculated that they have a role as pigments protecting the fungi from the light in the late phase of infection when the fungus can be exposed to light in its necrotrophic phase of life cycle. Furthermore, the metabolites could have antagonistic effects against competing fungi or bacteria. A full understanding of the ecological role of fungal secondary metabolites in the $V$. longisporum/B. napus system, can be achieved by their chemical characterization. 
This PhD thesis offers an established method for the isolation and purification of small molecules from xylem sap of $B$. napus, which can be applied to the purification of secondary metabolites from xylem sap from other host plants, but the improvement of xylem extraction for larger yield is still required. The thesis also suggests, an assay for testing the elements in the xylem sap for antimicrobial activities, this assay must be improved to better suit small molecule testing, but it can still be used in case of macromolecules in xylem sap. Finally the thesis reports changes in the metabolic profile of $V$. longisporum when VI-PKS-1 gene is RNAi silenced; and discusses the ecological role of the affected metabolites.

\section{Outlook}

The outlook of this work can be extended to

- Structure elucidation of the purified compound of the exact mass of 612.1197 Da, (in process), and the testing of the biological activity..

- Isolation and purification of secondary metabolites, which are present in the xylem sap of $B$. napus and specific for $V$. longisporum infection, followed by bioactivity testing of the isolated compounds.

- Examination of the macromolecules filtered from xylem sap for the presence of the known cruciferous phytoalexin detoxifying enzymes.

- Chemical investigation of secondary metabolites produced by $V$. longisporum and investigate their role in relation between $V$. longisporum/ $B$. napus. 


\section{References}

Beinhoff, M., 2011. Molecular and functional characterization of potential pathogenicity related genes from Verticillium longisporum. Georg-August-Universität Göttingen, Göttingen, Germany.

Bhat, R.G., Subbarao, K.V., 1999. Host Range Specificity in Verticillium dahliae. Phytopathology 89, 1218-1225.

De Wit, P.J.G.M., 2013. Microbial toxins in the green world. Fems Microbiol. Rev. 37, 1-2.

Dunker, S., Keunecke, H., Steinbach, P., Von Tiedemann, A., 2008. Impact of Verticillium longisporum on Yield and Morphology of Winter Oilseed Rape (Brassica napus) in Relation to Systemic Spread in the Plant. J. Phytopathol. 156, 698-707.

Eynck, C., Koopmann, B., Grunewaldt-Stoecker, G., Karlovsky, P., Tiedemann, A. von, 2007. Differential interactions of Verticillium longisporum and V. dahliae with Brassica napus detected with molecular and histological techniques. Eur. J. Plant Pathol. 118, 259274.

Eynck, C., Koopmann, B., Karlovsky, P., von Tiedemann, A., 2009. Internal Resistance in Winter Oilseed Rape Inhibits Systemic Spread of the Vascular Pathogen Verticillium longisporum. Phytopathology 99, 802-811.

Floerl, S., Druebert, C., Majcherczyk, A., Karlovsky, P., Kües, U., Polle, A., 2008. Defence reactions in the apoplastic proteome of oilseed rape (Brassica napus var. napus) attenuate Verticillium longisporum growth but not disease symptoms. Bmc Plant Biol. 8, 129.

Inderbitzin, P., Davis, R.M., Bostock, R.M., Subbarao, K.V., 2011. The Ascomycete Verticillium longisporum Is a Hybrid and a Plant Pathogen with an Expanded Host Range. Plos One 6, e18260. 
Johansson, A., Goud, J.-K.C., Dixelius, C., 2006. Plant Host Range of Verticillium longisporum and Microsclerotia Density in Swedish Soils. Eur. J. Plant Pathol. 114, 139149.

Kamble, A., Koopmann, B., von Tiedemann, A., 2012. Induced resistance to Verticillium longisporum in Brassica napus by $\beta$-aminobutyric acid. Plant Pathol. 62, 552-561.

Karapapa, V.K., Bainbridge, B.W., Heale, J.B., 1997. Morphological and molecular characterization of Verticillium longisporum comb, nov., pathogenic to oilseed rape. Mycol. Res. 101, 1281-1294.

Kehr, J., Buhtz, A., Giavalisco, P., 2005. Analysis of xylem sap proteins from Brassica napus. Bmc Plant Biol. 5, 11.

Kim, Y.-T., Lee, Y.-R., Jin, J., Han, K.-H., Kim, H., Kim, J.-C., Lee, T., Yun, S.-H., Lee, Y.W., 2005. Two different polyketide synthase genes are required for synthesis of zearalenone in Gibberella zeae. Mol. Microbiol. 58, 1102-1113.

Klosterman, S.J., Atallah, Z.K., Vallad, G.E., Subbarao, K.V., 2009. Diversity, Pathogenicity, and Management of Verticillium Species. Annu. Rev. Phytopathol. 47, 3962.

Kutchan, T.M., 2001. Ecological Arsenal and Developmental Dispatcher. The Paradigm of Secondary Metabolism. Plant Physiol. 125, 58-60.

Obermeier, C., Hossain, M.A., Snowdon, R., Knüfer, J., Tiedemann, A. von, Friedt, W., 2013. Genetic analysis of phenylpropanoid metabolites associated with resistance against Verticillium longisporum in Brassica napus. Mol. Breed. 1-15.

Pedras, M.S.C., Ahiahonu, P.W., 2002. Probing the phytopathogenic stem rot fungus with phytoalexins and analogues: unprecedented glucosylation of camalexin and 6methoxycamalexin. Bioorg. Med. Chem. 10, 3307-3312.

Pedras, M.S.C., Ahiahonu, P.W.K., 2005. Metabolism and detoxification of phytoalexins and analogs by phytopathogenic fungi. Phytochemistry 66, 391-411. 
Pedras, M.S.C., Chumala, P.B., Jin, W., Islam, M.S., Hauck, D.W., 2009. The phytopathogenic fungus Alternaria brassicicola: Phytotoxin production and phytoalexin elicitation. Phytochemistry 70, 394-402.

Pedras, M.S.C., Hossain, M., 2006. Metabolism of crucifer phytoalexins in Sclerotinia sclerotiorum: detoxification of strongly antifungal compounds involves glucosylation. Org. Biomol. Chem. 4, 2581-2590.

Pedras, M.S.C., Yaya, E.E., 2010. Phytoalexins from Brassicaceae: news from the front. Phytochemistry 71, 1191-7.

Pedras, M.S.C., Yaya, E.E., Glawischnig, E., 2011. The phytoalexins from cultivated and wild crucifers: chemistry and biology. Nat. Prod. Rep. 28, 1381-405.

Pedras, M.S.C., Zheng, Q.-A., Strelkov, S., 2008. Metabolic Changes in Roots of the Oilseed Canola Infected with the Biotroph Plasmodiophora brassicae: Phytoalexins and Phytoanticipins. J. Agric. Food Chem. 56, 9949-9961.

Pegg, G.F., Brady, B.L., 2002. Verticillium wilts 2002 pp. UK.

Ratzinger, A., 2008. Development and application of LC-MS-based differential metabolic profiling in plant systems. Georg-August-Universität Göttingen, Göttingen, Germany.

Ratzinger, A., Riediger, N., von Tiedemann, A., Karlovsky, P., 2009. Salicylic acid and salicylic acid glucoside in xylem sap of Brassica napus infected with Verticillium longisporum. J. Plant Res. 122, 571-579.

Ryang, S.H., Chung, S.Y., Lee, S.H., Cha, J.S., Yong Kim, H., Cho, T.J., 2002. Isolation of pathogen-induced Chinese cabbage genes by subtractive hybridization employing selective adaptor ligation. Biochem. Biophys. Res. Commun. 299, 352-359.

Siebold, M., Tiedemann, A. von, 2012. Potential effects of global warming on oilseed rape pathogens in Northern Germany. Fungal Ecol. 5, 62-72.

Springob, K., Kutchan, T.M., 2009. Introduction to the Different Classes of Natural Products, in: Osbourn, A.E., Lanzotti, V. (Eds.), Plant-derived Natural Products. Springer US, pp. 3-50. 
Watanabe, A., Fujii, I., Sankawa, U., Mayorga, M.E., Timberlake, W.E., Ebizuka, Y., 1999. Re-identification of Aspergillus nidulans wA gene to code for a polyketide synthase of naphthopyrone. Tetrahedron Lett. 40, 91-94.

Watanabe, C.M.H., Wilson, D., Linz, J.E., Townsend, C.A., 1996. Demonstration of the catalytic roles and evidence for the physical association of type I fatty acid synthases and a polyketide synthase in the biosynthesis of aflatoxin B1. Chem. Biol. 3, 463-469.

You, F., Han, T., Wu, J., Huang, B., Qin, L., 2009. Antifungal secondary metabolites from endophytic Verticillium sp. Biochem. Syst. Ecol. 37, 162-165. 


\section{Summary}

Verticillium longisporum is a devastating vascular pathogen which infects plants from Brassicacae including oilseed rape Brassica napus, the pathogen infects the plant through the root and enters the vascular system to which it is confined for most of its biotrophic part of life cycle. Bio-chemical interactions between the pathogen and the plants occur in the xylem vessels and specifically in the xylem sap. Small molecules in the xylem sap of oilseed rape Brassica napus are the topic of the two parts of this study. In the first chapter, isolation and purification of secondary metabolites which specifically occur in the xylem sap of $V$. longisporum-infected $B$. napus are described. By using a preparative HPLC system based on the analytical HPLC which detected these secondary metabolites, one of these secondary metabolites which has the exact mass of 612.1197 Da obtained. This compound was previously detected in positive and negative modes of ionization $\mathrm{m} / \mathrm{z} 613$ $[\mathrm{M}+\mathrm{H}]^{+}, m / z 611[\mathrm{M}-\mathrm{H}]^{-}$, respectively. The purified amount of the compound was not sufficient for complete structure elucidation. In the second chapter, a bio-assay was developed to study the inhibition effect of the small molecules present in xylem sap of $V . l o n g i s p o r u m$ infected $B$. napus plants on two microorganisms, the bacterium Agrobacterium tumefaciens and the yeast Saccharomyces sereviciae. An inhibition effect was observed on A.tumifaciens but not on $S$. cervesiae when the entire xylem sap of $B$. napus was used. The inhibition effect was negligible after removing the large molecules which have molecular weight larger than $3000 \mathrm{Da}$; therefore, small molecules in xylem sap have no or a negligible inhibition effect on $A$. tumifaciens. In the third chapter, comparative metabolic profiling of supernatant of wild type $V$. longisporum grown on Potato dextrose broth (PDB) medium was compared to mutant type resulted from silencing VI-PKS-1, a gene encode to polyketide synthase in $V$. longisporum. The study was conducted using chemical analytical techniques (HPLC combined with mass spectrometry (MS), Diode array detection (DAD), UV Time-of-flight mass spectrometry (TOFMS.) As a result, several metabolites were found in the supernatant of $V$. longisporum wild type strain which were not present or present in lower amounts in the supernatant of VI-PKS-1 silenced type. 


\section{Acknowledgments}

I would like to thank Prof. Dr. Petr Karlovsky for giving me the opportunity to conduct my PhD research work in his laboratory, and for his supervision and continuous support during my studies and for his valuable remarks and suggestions which were very helpful in accomplishing the aim of my PhD work.

Sincere thanks to The German Academic Exchange Service (DAAD) for granting me a scholarship which made it possible to continue my PhD studies in Germany.

I would like to thank Prof. Dr. Andreas von Tiedemann for his co-supervision of my PhD thesis.

My sincere thanks to Dr. Kirstin Feussner and Dr. Cornelia Herrfurth from the department of the biochemistry for their kind cooperation and the support while conducting a part of my work in their department and to Prof. Dr. Ivo Feussner for the fruitful collaboration a part of the work in his lab.

I would like thank my colleagues and the members of the molecular phytopathology and mycotoxin research group especially, Katharina, Ruth, Heike, Patricia and all the other members for their help and support during my studies in the department.

My sincere gratitude to my family in Syria who suffer there while I am absent from them, I really miss you, and my heart is with you. My Dad whom I have not seen for over five years and my Mom, I really miss your hug.

My thanks go also to all my friends in Göttingen, which made my stay in this lovely city a wonderful experience.

Husam

Göttingen 28.05.2013 


\section{Curriculum vitae}

\section{Personal details}

$\begin{array}{ll}\text { Name: } & \text { Husam } \\ \text { Surname: } & \text { Ibrahem Aroud } \\ \text { Date of Birth: } & \mathbf{1 3 . 0 4 . 1 9 7 8} \\ \text { Place of Birth: } & \text { Damascus, Syria } \\ \text { Marital Status: } & \text { Single } \\ \text { Nationality: } & \text { Syrian }\end{array}$

\section{Education}

[Nov. 2007 - Jul. 2013] Ph.D student in Georg August University of Goettingen, Department of Crop Sciences, Molecular Phytopathology \& Mycotoxin Research.

[Oct. 2004 - Feb. 2007] Mediterranean Agronomic Institute of Chania (MAICh), Chania, Greece. [Master of Science M.Sc in Natural products and Biotechnology]

[Oct. 2004 - Jul. 2005] Mediterranean Agronomic Institute of Chania (MAICh), Chania, Greece. [Post Graduate Diploma (D.S.P.U) in Natural Products and Biotechnology]

[Oct. 2002 - Sept. 2004] University of Aleppo, Second faculty of Agriculture, Aleppo, Syria. [Post graduate diploma in Plant Protection]

[Sept. 1997- Sept. 2002] University of Aleppo, Second faculty of Agriculture, Aleppo, Syria. [B.Sc in Agricultural engineering, specialization: Plant Protection] 


\section{Publications}

Floerl S, Druebert C, Aroud HI, Karlovsky P, Polle A (2010): Disease symptoms and mineral nutrition in Arabidopsis thaliana in response to Verticillium longisporum VI43 infection. Journal of Plant Pathology 92: 695-702.

\section{Posters}

Purification and chemical characterization of secondary metabolites from xylem sap of Brassica napus infected with Verticillium longisporum. H. I. Aroud, A. Ratzinger and P. Karlovsky. (The $8^{\text {th }}$ annual meeting of Metabolomics Society) 25. - 28. Jun. 2012, Washington DC, USA.

Apoplastic proteins of Brassica napus var. napus possess antimicrobial activity C. Druebert, H.I. Aroud, G. Lohaus, P. Karlovsky and A. Polle. (From Foliar to Root-Interacting Pathogens and Symbionts) 16. - 18. Feb. 2011. Göttingen, Germany.

Detection and functional analysis of a polyketide synthase gene in Verticillium longisporum. M. Beinhoff, H. I. Aroud, M. Quambusch, W. Hiegl, H. Xu and P. Karlovsky. (From Foliar to Root-Interacting Pathogens and Symbionts) 16. - 18. Feb. 2011, Göttingen, Germany. 


\section{Declarations}

1. I, hereby, declare that this Ph.D. dissertation has not been presented to any other examining body either in its present or a similar form.

Furthermore, I also affirm that I have not applied for a Ph.D. at any other higher school of education.

Göttingen, 28.05.2013

HUSAM IBRAHEM AROUD

2. I, hereby, solemnly declare that this dissertation was undertaken independently and without any unauthorised aid.

Göttingen, 28.05.2013 